\title{
Spectral theory of no-pair Hamiltonians
}

\author{
Oliver Matte and Edgardo Stockmeyer \\ Mathematisches Institut der Universität München \\ Theresienstraße 39, D-80333 München, Germany.
}

\begin{abstract}
We prove a HVZ theorem for a general class of no-pair Hamiltonians describing an atom or positively charged ion with several electrons in the presence of a classical external magnetic field. Moreover, we show that there exist infinitely many eigenvalues below the essential spectrum and that the corresponding eigenfunctions are exponentially localized. The novelty is that the electrostatic and magnetic vector potentials as well as a non-local exchange potential are included in the projection determining the model. As a main technical tool we derive various commutator estimates involving spectral projections of Dirac operators with external fields. Our results apply to all nuclear charges $Z \leqslant 137$.
\end{abstract}

Keywords: Dirac operator, Brown and Ravenhall, no-pair operator, pseudorelativistic, Furry picture, intermediate pictures, HVZ theorem, exponential localization.

\section{Introduction}

The relativistic dynamics of a single electron moving in the potential of a static nucleus, $V_{\mathrm{C}} \leqslant 0$, in the presence of an external classical magnetic field $B=\operatorname{curl} A$ is generated by the Dirac operator 1

$$
D_{A, V_{\mathrm{C}}}:=\alpha \cdot(-i \nabla+A)+V_{\mathrm{C}}
$$

Here an electron is a state lying in the positive spectral subspace of $D_{A, V_{\mathrm{C}}}$. A ground state of the one-electron atom modeled by $D_{A, V_{\mathrm{C}}}$ can be characterized

1 Energies are measured in units of $m c^{2}, m$ denoting the rest mass of an electron and $c$ the speed of light. Length are measured in units of $\hbar /(m c)$, which is the Compton wave length divided by $2 \pi$. $\hbar$ is Planck's constant divided by $2 \pi$. In these units the square of the elementary charge equals the fine structure constant, $e^{2} \approx 1 / 137.037$. 
as an energy minimizing bound state of the restriction of $D_{A, V_{\mathrm{C}}}$ to its positive spectral subspace, $\Lambda_{A, V_{\mathrm{C}}}^{+} L^{2}\left(\mathbb{R}^{3}, \mathbb{C}^{4}\right)$, where

$$
\Lambda_{A, V_{\mathrm{C}}}^{+}=\frac{1}{2}+\frac{1}{2} \operatorname{sgn}\left(D_{A, V_{\mathrm{C}}}\right) .
$$

This is confirmed by Dirac's interpretation of the negative spectral subspace as a completely filled sea of virtual electrons which, on account of Pauli's exclusion principle, forces an additional electron to attain a state of positive energy. On the other hand it is well-known that there is no canonical à-priori given atomic Hamiltonian generating the relativistic time evolution of $N>1$ interacting electrons in the potential landscape of $V_{\mathrm{C}}$ under the influence of $B$. Guided by non-relativistic quantum mechanics one might naively propose to start with the formal expression

$$
\sum_{j=1}^{N} D_{A, V_{\mathrm{C}}}^{(j)}+\sum_{1 \leqslant j<k \leqslant N} W_{j k}
$$

where the superscript $(j)$ means that the operator below it acts on the $j^{\text {th }}$ electron and $W_{j k} \geqslant 0$ is the interaction potential between the $j^{\text {th }}$ and $k^{\text {th }}$ electron. It then turns out, however, that (3) suffers from the phenomenon of continuum dissolution, that is, the eigenvalue problem associated to (3) has no normalizable solutions [35]. A frequently used ansatz to find a reasonable and semi-bounded Hamiltonian for an $N$-electron atomic system again incorporates the concept of a Dirac sea. Namely, one projects (3) onto the $N$-fold antisymmetric tensor product of a suitable one-electron subspace, i.e., one considers operators of the form

$$
H_{N}:=\Lambda_{A, V}^{+, N}\left(\sum_{j=1}^{N} D_{A, V_{\mathrm{C}}}^{(j)}+\sum_{1 \leqslant j<k \leqslant N} W_{j k}\right) \Lambda_{A, V}^{+, N}
$$

where

$$
\Lambda_{A, V}^{+, N}:=\bigotimes_{j=1}^{N} \Lambda_{A, V}^{+(j)}
$$

Here $\Lambda_{A, V}^{+}$is defined as in (1) \&(2) but with $V_{\mathrm{C}}$ replaced by a new potential $V$. We emphasize that $H_{N}$ can formally be derived from quantum electrodynamics by a procedure that neglects the creation and annihilation of electron-positron pairs [34], the latter being defined with respect to $\Lambda_{A, V}^{+}$. Therefore, operators of the form (41) are often called no-pair Hamiltonians. Obviously, the question arises how to choose the new potential $V$ determining the projection (5) or, in 
other words, how to fix the Dirac sea for one electron in the presence of the others in a physically efficient way. Various possibilities are discussed from a physical and numerical point of view in [34, 35]. The choice $V=0$ is refered to as the free picture, or Brown-Ravenhall model [9]. $\left(H_{1}\right.$ is also sometimes called the Bethe-Salpeter operator in this case [7].) It has by now been studied in many mathematical works $[2,3,44,5,10,14,18,17,21,22,23,27,28,29,30,37$, 38, 39]. This is due to the fact that the free projection, $\Lambda_{0,0}^{+}$, can be calculated explicitely both in momentum and position space [2, 30]. The stability of the second kind in the free picture with quantized electro-magnetic field (also included in the projection) is treated in [26]. The free picture is considered as one extreme case in a family of intermediate pictures. The opposite extreme case, called the Furry picture, is given by substituting the (negative) Coulomb potential $V_{\mathrm{C}}$ for $V$. Other members of that family are obtained by choosing $V$ to be equal to $V_{\mathrm{C}}$ plus some additional positive and in general non-local operator. The additional non-local term may incorporate the interaction with the remaining electrons and also take vacuum polarization effects into account; compare [19] where similar projections appear in a somewhat different setting. We remark that the Furry and intermediate pictures give better numerical results in comparison to the free picture [34, 35].

In this paper we do not aim to contribute to the subtle question of optimizing the choice of $V$. Rather we consider a general class of potentials which can be written as $V=V_{\mathrm{C}}+V_{\mathrm{H}}+V_{\mathrm{E}}$, where $V_{\mathrm{C}}$ may have several Coulomb-type singularities, $V_{\mathrm{H}}$ is a bounded potential function vanishing at infinity, and $V_{\mathrm{E}}$ is a compact non-local operator that behaves nicely under conjugation with exponential weights. Our goal then is to establish some basic qualitative spectral properties of $H_{N}$. First, we show that $H_{N}$ is well-defined on a natural dense subspace (which is not obvious) and, thus, has a self-adjoint Friedrichs extension. We further locate its essential spectrum and, assuming that the number of electrons does not exceed the sum of all nuclear charges, we prove that there exist infinitely many eigenvalues below the ionization threshold. Moreover, we show that the corresponding eigenfunctions are exponentially localized. Our results apply to all nuclear charges $Z \leqslant 137$. The 'easy part' of the HVZ theorem, i.e., the upper bound on the ionization threshold holds for a certain class of possibly unbounded magnetic fields. The 'hard part', i.e., the lower bound on the ionization threshold as well as our results on existence of eigenvectors and their exponential localization are derived for bounded magnetic fields.

As an essential and novel technical input, necessary to obtain any of the results mentioned above, we derive various commutator estimates involving the spectral projection $\Lambda_{A, V}^{+}$, exponential weights, and cut-off functions. They describe the non-local properties of $\Lambda_{A, V}^{+}$in an $L^{2}$-sense and might be of inde- 
pendent interest.

We remark that our commutator estimates would also allow to analyze the Hamitonian $H_{N}$ in the free picture proceeding along the discussion of the Furry and intermediate pictures presented here. There would, however, appear a subtlety right in the beginning of the analysis: Namely, for vanishing magnetic fields, it is known that the one-particle Brown-Ravenhall operator is stable if and only if $Z \leqslant Z_{c}:=\frac{1}{e^{2}} \frac{2}{(2 / \pi)+(\pi / 2)} \approx 124.2$ [14]. For non-zero magnetic fields, such a critical value for the nuclear charge is not known in general [27] and one had to study this question first or to introduce the existence of a critical nuclear charge for $H_{1}$ as a hypothesis. What is known is that in the presence of an exterior $B$-field one must include the vector potential in the projection for otherwise the model is always unstable if $N>1$ [18]. (More precisely, the sum of the quadratic form and the field energy is unbounded from below if the magnetic field is also considered as a variable in the minimization.)

Finally, we comment on some closely related recent work. In the free picture and for vanishing exterior magnetic field, an HVZ theorem and the existence of infinitely many eigenvalues below the essential spectrum have been proved in [30], for nuclear charges $Z \leqslant Z_{c}$. The case $N=2$ is also treated in [22]. A more general HVZ theorem that applies to different particle species and a wider class of interaction potentials and exterior fields in the free picture has been established very recently in [28]. Moreover, the reduction to irreducible representations of the groups of rotation and reflection and permutations of identical particles is considered in [28]. The exponential localization of eigenvectors in the Brown-Ravenhall model is studied in [29] improving and generalizing earlier results from [2]. In all these works the authors employ explicite position space representations of $\Lambda_{0,0}^{+}$. An HVZ theorem in the free picture with constant magnetic field is established in [23] again using explicite representations for the projection based on Mehler's formula. By employing somewhat more abstract arguments we are able to study commutator estimates for a wider class of projections in this paper.

The article is organized as follows. In Section 2 we introduce our mathematical model precisely and state our main theorems. Section 3 is devoted to the study of some non-local properties of $\Lambda_{A, V}^{+}$expressed in terms of various commutator estimates which form the basis of the spectral analysis of $H_{N}$. In Section 4 we derive the exponential localization estimate for $H_{N}$ and in Section 5 infer a lower bound on the threshold energy. Section 6 deals with Weyl sequences and, finally, in Section 7 we show that $H_{N}$ possesses infinitely many eigenvalues below the threshold energy. The main text is followed by an appendix containing the proofs of some results of Section 3 . 
Some frequently used notation. Open balls in $\mathbb{R}^{3}$ with radius $R>0$ and center $z \in \mathbb{R}^{3}$ are denoted by $\mathcal{B}_{R}(z)$. Spectral projections of a self-adjoint operator, $T$, on some Hilbert space are denoted by $E_{\lambda}(T)$ and $E_{I}(T)$, if $\lambda \in \mathbb{R}$ and $I$ is an interval. $\mathcal{D}(T)$ denotes the domain of the operator $T$ and $\mathcal{Q}(T)$ its form domain. The characteristic function of a subset $\mathcal{M} \subset \mathbb{R}^{n}$ is denoted by $\mathbb{1}_{\mathcal{M}}$. $C, C^{\prime}, C^{\prime \prime}, \ldots$ denote constants whose values might change from one estimate to another.

\section{The model and main results}

In our choice of units the free Dirac operator reads

$$
D_{0}:=-i \alpha \cdot \nabla+\beta:=-i \sum_{j=1}^{3} \alpha_{j} \partial_{x_{j}}+\beta .
$$

Here $\alpha=\left(\alpha_{1}, \alpha_{2}, \alpha_{3}\right)$ and $\beta=: \alpha_{0}$ are $4 \times 4$ hermitian matrices which satisfy the Clifford algebra relations

$$
\left\{\alpha_{i}, \alpha_{j}\right\}=2 \delta_{i j} \mathbb{1}, \quad 0 \leqslant i, j \leqslant 3 .
$$

In Dirac's representation, which we fix throughout the paper, they are given as

$$
\alpha_{j}=\left(\begin{array}{rr}
0 & \sigma_{j} \\
\sigma_{j} & 0
\end{array}\right), \quad j=1,2,3, \quad \beta=\left(\begin{array}{rr}
\mathbb{1} & 0 \\
0 & -\mathbb{1}
\end{array}\right)
$$

where $\sigma_{1}, \sigma_{2}, \sigma_{3}$ are the standard Pauli matrices. $D_{0}$ is a self-adjoint operator in the Hilbert space

$$
\mathscr{H}:=L^{2}\left(\mathbb{R}^{3}, \mathbb{C}^{4}\right)
$$

with domain $H^{1}\left(\mathbb{R}^{3}, \mathbb{C}^{4}\right)$. Its spectrum is purely absolutely continuous and given by the union of two halflines,

$$
\sigma\left(D_{0}\right)=\sigma_{\mathrm{ac}}\left(D_{0}\right)=(-\infty,-1] \cup[1, \infty) .
$$

Next, we formulate our precise hypotheses on the exterior electrostatic potential $V_{\mathrm{C}}$ and on the potential $V$ determining the Dirac sea. We think that, at least with regards to the commutator estimates in Section 3, it is interesting to keep the conditions on $V_{\mathrm{C}}$ and $V$ fairly general.

1 Hypothesis. There are a finite set $\mathcal{Y} \subset \mathbb{R}^{3}$, \#Y $<\infty$, such that $V_{\mathrm{C}} \in$ $L_{\text {loc }}^{\infty}\left(\mathbb{R}^{3} \backslash \mathcal{Y}, \mathscr{L}\left(\mathbb{C}^{4}\right)\right)$ is almost everywhere hermitian and

$$
\left\|V_{\mathrm{C}}(x)\right\| \longrightarrow 0, \quad|x| \rightarrow \infty .
$$


Moreover, there exist $\gamma \in(0,1)$ and $\varepsilon>0$ such that the balls $\mathcal{B}_{\varepsilon}(y), y \in \mathcal{Y}$, are mutually disjoint and, for $0<|x|<\varepsilon$ and $y \in \mathcal{Y}$,

$$
\left\|V_{\mathrm{C}}(y+x)\right\| \leqslant \frac{\gamma}{|x|} .
$$

2.1 Example. The main example for a potential satisfying Hypothesis 1 is certainly the Coulomb potential generated by a finite number of static nuclei,

$$
V_{\mathrm{C}}(x)=-\sum_{y \in \mathcal{Y}} \frac{e^{2} Z_{y}}{|x-y|} \mathbb{1}, \quad x \in \mathbb{R}^{3} \backslash \mathcal{Y} .
$$

In this case the restriction on the strength of the singularities of $V_{\mathrm{C}}$ imposed in Hypothesis 1 allows for all nuclear charges, $Z_{y} \in \mathbb{N}, Z_{y} \leqslant 137$, for $y \in \mathcal{Y}$.

2 Hypothesis. $V=V_{\mathrm{C}}+V_{\mathrm{H}}+V_{\mathrm{E}}$, where $V_{\mathrm{C}}$ fulfills Hypothesis 1 and $V_{\mathrm{H}} \in$ $L_{\text {loc }}^{\infty}\left(\mathbb{R}^{3}, \mathscr{L}\left(\mathbb{C}^{4}\right)\right)$ is an almost everywhere hermitian matrix-valued function dropping off to zero at infinity,

$$
\left\|V_{\mathrm{H}}(x)\right\| \longrightarrow 0, \quad|x| \rightarrow \infty .
$$

$V_{\mathrm{E}}$ is compact and has the following property: There exist $m>0$ and some increasing function $c:[0, m) \rightarrow(0, \infty)$ such that, for every $F \in C^{1}\left(\mathbb{R}^{3}, \mathbb{R}\right)$ with $|\nabla F| \leqslant a<m$,

$$
\begin{aligned}
\forall \chi \in C^{1}\left(\mathbb{R}^{3},[0,1]\right):\left\|\left[e^{F} V_{\mathrm{E}} e^{-F}, \chi\right]\right\| & \leqslant c(a)\|\nabla \chi\|_{\infty}, \\
\left\|\left[V_{\mathrm{E}}, e^{F}\right] e^{-F}\right\| & \leqslant c(a)\|\nabla F\|_{\infty}, \\
\lim _{R \rightarrow \infty}\left\|\mathbb{1}_{\mathbb{R}^{3} \backslash \mathcal{B}_{R}(0)} e^{F} V_{\mathrm{E}} e^{-F} \mathbb{1}_{\mathbb{R}^{3} \backslash \mathcal{B}_{R}(0)}\right\| & =0 .
\end{aligned}
$$

2.2 Example. (i) Possible choices for $V_{\mathrm{H}}$ and $V_{\mathrm{E}}$ satisfying the conditions of Hypothesis 2 are the Hartee and non-local exchange potentials corresponding to a set of exponentially localized orbitals $\varphi_{1}, \ldots, \varphi_{M} \in \mathscr{H},\left|\varphi_{i}(x)\right| \leqslant C e^{-m|x|}$, $1 \leqslant i \leqslant M$, for some $C \in(0, \infty)$. Their Hartee potential is given as

$$
V_{\mathrm{H}}(x):=e^{2} \sum_{i=1}^{M}\left(\left|\varphi_{i}\right|^{2} * \frac{1}{|\cdot|}\right)(x), \quad x \in \mathbb{R}^{3} .
$$

It incorporates the presence of $M$ electrons in a fixed state into the Dirac sea by a smeared out background density. The exchange potential corresponding to $\varphi_{1}, \ldots, \varphi_{M}$ is the integral operator with matrix-valued kernel

$$
V_{\mathrm{E}}(x, y):=e^{2} \sum_{i=1}^{M} \frac{\varphi_{i}(x) \varphi_{i}^{*}(y)}{|x-y|} .
$$


It is a correction to the Hatree potential accounting for the Pauli principle. In the sense of quadratic forms it then holds $V_{\mathrm{C}} \leqslant V=V_{\mathrm{C}}+V_{\mathrm{H}}+V_{\mathrm{E}} \leqslant 0$, which justifies the notion 'intermediate picture'.

(ii) More generally, we may set $V_{\mathrm{H}}:=\varrho *|\cdot|^{-1}$, for some $0 \leqslant \varrho \in L^{1} \cap L^{5 / 3}\left(\mathbb{R}^{3}\right)$. In this case we find some $C \in(0, \infty)$ such that $0 \leqslant V_{\mathrm{H}} \leqslant C /|\cdot|$. Moreover, standard theorems on integral operators show that every kernel with values in the set of hermitian $(4 \times 4)$-matrices satisfying

$$
\left\|V_{\mathrm{E}}(x, y)\right\| \leqslant C^{\prime} \frac{e^{-m|x-y|}}{\langle x\rangle^{\rho}|x-y|\langle y\rangle^{\rho}},
$$

for some $m, \rho, C^{\prime}>0$, yields a compact operator satisfying the conditions of Hypothesis 2 .

As a first consequence of Hypothesis 2 we find, for every locally bounded vector potential $A: \mathbb{R}^{3} \rightarrow \mathbb{R}^{3}$, a distinguished self-adjoint realization of the Dirac operator

$$
D_{A, V}=\alpha \cdot(-i \nabla+A)+\beta+V,
$$

whose essential spectrum is again contained in $(-\infty,-1] \cup[1, \infty)$; see Lemma 3.2 below, where we recall some important well-known facts on Dirac operators with singular potentials. Therefore, it makes sense to define the spectral projections,

$$
\Lambda_{A, V}^{+}:=E_{\left[e_{0}, \infty\right)}\left(D_{A, V}\right), \quad \Lambda_{A, V}^{-}:=\mathbb{1}-\Lambda_{A, V}^{+}
$$

where

$$
e_{0} \in \varrho\left(D_{A, V}\right) \cap(-1,1) .
$$

For later reference we introduce the parameter

$$
\triangle_{0}:=\min \left\{1-e_{0}, e_{0}+1\right\} .
$$

Many of our technical results on $D_{A, V}$, for instance, various commutator estimates of Section 3 hold actually true under the mere assumption that the components of $A$ are locally bounded. Of course, if not all eigenvalues of $D_{A, V}$ are larger than -1 and $e_{0}$ is chosen between -1 and the lowest eigenvalue, the physical relevance of the $N$-particle Hamiltonian $H_{N}$ becomes rather questionable. We remark that such situations are not excluded by our hypotheses. For instance, if $V_{\mathrm{C}}$ is the Coulomb potential and the intensity of a constant exterior magnetic field is increased, then the lowest eigenvalue of $D_{A, V_{\mathrm{C}}}$ eventually delves into the lower continuum [13]. Nevertheless, our theorems hold for any choice of $e_{0}$ as in (15). 
In order to define the atomic no-pair Hamiltonian precisely we first set

$$
\mathscr{H}_{N}:=\bigotimes_{i=1}^{N} \mathscr{H}, \quad \mathscr{H}_{N}^{+}:=\Lambda_{A, V}^{+, N} \mathscr{H}_{N}, \quad N \in \mathbb{N}, \quad \mathscr{H}^{+}:=\mathscr{H}_{1}^{+}
$$

where $\Lambda_{A, V}^{+, N}$ is given by (15) and (14). We let $W: \mathbb{R}^{3} \times \mathbb{R}^{3} \rightarrow[0, \infty]$ denote the interaction potential between two electrons.

3 Hypothesis. There is some $\widetilde{\gamma}>0$ such that, for all $x, y \in \mathbb{R}^{3}, x \neq y$,

$$
0 \leqslant W(x, y)=W(y, x) \leqslant \widetilde{\gamma}|x-y|^{-1} .
$$

When we consider $N$ electrons located at $x_{1}, \ldots, x_{N} \in \mathbb{R}^{3}$ we denote their common position variable by $X=\left(x_{1}, \ldots, x_{N}\right)$. Furthermore, we often write $W_{j k}$ for the maximal multiplication operator in $\mathscr{H}_{N}$ induced by the function $\left(\mathbb{R}^{3}\right)^{N} \ni X \mapsto W\left(x_{j}, x_{k}\right)$. For $N \in \mathbb{N}$, we introduce a symmetric, semi-bounded operator acting in $\mathscr{H}_{N}^{+}$by

$$
\begin{aligned}
\mathcal{D}\left(\stackrel{\circ}{H}_{N}\right) & :=\Lambda_{A, V}^{+, N} \mathscr{D}_{N}, \quad \mathscr{D}_{N}:=\bigotimes_{i=1}^{N} \mathscr{D}, \quad \mathscr{D}:=C_{0}^{\infty}\left(\mathbb{R}^{3}, \mathbb{C}^{4}\right) \\
\stackrel{\circ}{H}_{N} \Phi & :=\Lambda_{A, V}^{+, N}\left(\sum_{i=1}^{N} D_{A, V_{\mathrm{C}}}^{(i)}+\sum_{1 \leqslant i<j \leqslant N} W_{i j}\right) \Lambda_{A, V}^{+, N} \Phi, \quad \Phi \in \mathcal{D}\left(\stackrel{\circ}{H}_{N}\right) .
\end{aligned}
$$

2.3 Proposition. Assume that $V$ fulfills Hypothesis 2, $W$ fulfills Hypothesis 3 , and that $A \in L_{\text {loc }}^{\infty}\left(\mathbb{R}^{3}, \mathbb{R}^{3}\right)$ satisfies $\left\|e^{-\tau_{0}|x|} A\right\|_{\infty}<\infty$, for some $0 \leqslant \tau_{0}<$ $\min \left\{\triangle_{0}, m\right\}$. Then the operator $\stackrel{\circ}{H}_{N}$ given by (18) and (19) is well-defined, symmetric, and semi-bounded from below.

Proof: The only claim that is not obvious is that $W_{i j} \Lambda_{A, V}^{+, N} \phi$ is again squareintegrable, for every $\phi \in \mathscr{D}_{N}$. This follows, however, from Corollary 3.10 .

We denote the Friedrichs extension of $\stackrel{\circ}{H}_{N}$ by $H_{N}$. Note that we do not require the elements in the domain of $H_{N}$ to be anti-symmetric since in our proofs it is convenient to consider $H_{N}$ as an operator acting in the full tensor product. Of course, in the end we shall be interested in the restriction of $H_{N}$ to the anti-symmetric (fermionic) subspace of $\mathscr{H}_{N}^{+}$. We denote the antisymmetrization operator on $\mathscr{H}_{N}$ by $\mathcal{A}_{N}$,

$$
\left(\mathcal{A}_{N} \Phi\right)(X)=\frac{1}{N !} \sum_{\sigma \in \mathfrak{S}_{N}} \operatorname{sgn}(\sigma) \Phi\left(x_{\sigma(1)}, \ldots, x_{\sigma(N)}\right), \quad \Phi \in \mathscr{H}_{N}
$$


where $\mathfrak{S}_{N}$ is the group of permutations of $\{1, \ldots, N\}$, and define the no-pair Hamiltonian by

$$
H_{N}^{\mathcal{A}}:=H_{N} \uparrow_{\mathcal{A}_{N} \mathscr{H}_{N}^{+}} .
$$

Our first main result is the following theorem, where

$$
\mathscr{E}_{N}^{\mathcal{A}}:=\inf \sigma\left(H_{N}^{\mathcal{A}}\right), \quad N \in \mathbb{N}, \quad \mathscr{E}_{0}^{\mathcal{A}}:=0 .
$$

2.4 Theorem. (Exponential localization) Assume that $V$ and $W$ fulfill Hypotheses 2 and 3, respectively. If $A \in C^{1}\left(\mathbb{R}^{3}, \mathbb{R}^{3}\right)$ and $B=\operatorname{curl} A$ is bounded and if $I \subset \mathbb{R}$ is an interval with $\sup I<\mathscr{E}_{N-1}^{\mathcal{A}}+1$, then there exists $b \in(0, \infty)$ such that $\operatorname{Ran}\left(E_{I}\left(H_{N}^{\mathcal{A}}\right)\right) \subset \mathcal{D}\left(e^{b|X|}\right)$ and

$$
\left\|e^{b|X|} E_{I}\left(H_{N}^{\mathcal{A}}\right)\right\|<\infty .
$$

Proof: This theorem is proved in Section 4 .

2.5 Remark. In the case $N=1$ the assertion of Theorem 2.4 still holds true under the assumptions of Proposition 2.3. This follows from the proof of Theorem 2.4. In fact, for $N=1$, we do not have to control error terms involving the interaction $W$ which is the only reason why $B$ is assumed to be bounded in Theorem 2.4. If also $V=V_{\mathrm{C}}$, then we obtain an exponential localization estimate for an eigenvector, $\phi_{E}$, with eigenvalue $E \in(-1,1)$ of the Dirac operator $D_{A, V_{\mathrm{C}}}$. The estimate on the decay rate which could be extracted from our proof is, however, suboptimal due to error terms coming from the projections; see also [6] for decay estimates for Dirac operators.

Next, we introduce a hypothesis which is used to prove the 'easy part' of the HVZ theorem below.

4 Hypothesis. (i) For every $\lambda \geqslant 1$, there exist radii, $1 \leqslant R_{1}<R_{2}<\ldots$, $R_{n} \nearrow \infty$, and $\psi_{1}, \psi_{2}, \ldots \in \mathscr{D}$ such that

$$
\left\|\psi_{n}\right\|=1, \quad \operatorname{supp}\left(\psi_{n}\right) \subset \mathbb{R}^{3} \backslash \mathcal{B}_{R_{n}}(0), \quad \lim _{n \rightarrow \infty}\left\|\left(D_{A}-\lambda\right) \psi_{n}\right\|=0 .
$$

(ii) $A \in C^{1}\left(\mathbb{R}^{3}, \mathbb{R}^{3}\right)$ and $B=\operatorname{curl} A$ has the following property: There are $b_{1} \in(0, \infty)$ and $0 \leqslant \tau<\min \left\{\triangle_{0}, m\right\}$ ( $m$ and $\triangle_{0}$ are the parameters appearing in Hypothesis 2 and (16) ) such that, for all $x, y \in \mathbb{R}^{3}$,

$$
|B(x)-B(y)| \leqslant b_{1} e^{\tau|x-y|} .
$$


2.6 Example. ([20]) We recall a result from [20] which provides a large class of examples where Hypothesis 4 (i) is fulfilled: Suppose that $A \in C^{\infty}\left(\mathbb{R}^{3}, \mathbb{R}^{3}\right)$, $B=\operatorname{curl} A$, and set, for $x \in \mathbb{R}^{3}$ and $\nu \in \mathbb{N}$,

$$
\epsilon_{0}(x):=|B(x)|, \quad \epsilon_{\nu}(x):=\frac{\sum_{|\alpha|=\nu}\left|\partial^{\alpha} B(x)\right|}{1+\sum_{|\alpha|<\nu}\left|\partial^{\alpha} B(x)\right|} .
$$

Suppose further that there exist $\nu \in \mathbb{N}_{0}, z_{1}, z_{2}, \ldots \in \mathbb{R}^{3}$, and $\rho_{1}, \rho_{2}, \ldots>0$ such that $\rho_{n} \nearrow \infty$, the balls $\mathcal{B}_{\rho_{n}}\left(z_{n}\right), n \in \mathbb{N}$, are mutually disjoint and

$$
\sup \left\{\epsilon_{\nu}(x) \mid x \in \mathcal{B}_{\rho_{n}}\left(z_{n}\right)\right\} \longrightarrow 0, \quad n \rightarrow \infty
$$

Then there is a Weyl sequence, $\psi_{1}, \psi_{2}, \ldots$, that satisfies the conditions of Hypothesis $4(\mathrm{i})$.

The fact that the additional assumption of Part (ii) of the next theorem yields a lower bound on the ionization threshold is an observation made in [16] for Schrödinger operators.

2.7 Theorem. (HVZ) Assume that $V$ and $W$ fulfill Hypotheses 2 and 3 , respectively. Then the following assertions hold true:

(i) If Hypothesis 4 is fulfilled also, then $\sigma_{\text {ess }}\left(H_{N}^{\mathcal{A}}\right) \supset\left[\mathscr{E}_{N-1}^{\mathcal{A}}+1, \infty\right)$.

(ii) Assume additionally that, for every interval $I \subset \mathbb{R}$ with $\sup I<\mathscr{E}_{N-1}^{\mathcal{A}}+1$, there is some $g \in C(\mathbb{R},(0, \infty))$ such that $g(r) \rightarrow \infty$, as $r \rightarrow \infty$, and $g(|X|) E_{I}\left(H_{N}^{\mathcal{A}}\right) \in \mathscr{L}\left(\mathcal{A}_{N} \mathscr{H}_{N}\right)$. Then $\sigma_{\text {ess }}\left(H_{N}^{\mathcal{A}}\right) \subset\left[\mathscr{E}_{N-1}^{\mathcal{A}}+1, \infty\right)$. In particular, this inclusion is valid if $A \in C^{1}\left(\mathbb{R}^{3}, \mathbb{R}^{3}\right)$ and $B=\operatorname{curl} A$ is bounded.

Proof: (i) follows directly from Lemma 6.3 and (ii) follows from Theorem 5.1 and Theorem 2.4 .

To show the existence of infinitely many eigenvalues below the bottom of the essential spectrum of $H_{N}^{\mathcal{A}}$ we certainly need a condition on the relationship between $V_{\mathrm{C}}, W$, and the magnetic field. To formulate it we set, for $\delta, R>0$,

$$
\begin{aligned}
S_{\delta}(R) & :=\left\{x \in \mathbb{R}^{3}:(1-\delta) R \leqslant|x| \leqslant(1+\delta) R\right\} \\
v_{\star}(\delta, R) & :=\sup _{x \in S_{\delta}(R)} \sup _{|v|=1}\left\langle v \mid V_{\mathrm{C}}(x) v\right\rangle_{\mathbb{C}^{4}} .
\end{aligned}
$$


5 Hypothesis. (i) $V$ fulfills Hypothesis 2.

(ii) $A \in C^{1}\left(\mathbb{R}^{3}, \mathbb{R}^{3}\right)$ and $B=\operatorname{curl} A$ is bounded.

(iii) There exist radii $1 \leqslant R_{1}<R_{2}<\ldots, R_{n} \nearrow \infty$, some constant $\delta \in$ $(0,1 / N)$, and a sequence of spinors, $\psi_{1}, \psi_{2}, \ldots \in \mathscr{D}$, with vanishing lower spinor components, $\psi_{n}=\left(\psi_{n, 1}, \psi_{n, 2}, 0,0\right)^{\top}, n \in \mathbb{N}$, such that

$$
\left\|\psi_{n}\right\|=1, \quad \operatorname{supp}\left(\psi_{n}\right) \subset\left\{R_{n}<|x|<(1+\delta / 2) R_{n}\right\}, \quad 2 R_{n} \leqslant R_{n+1},
$$

for all $n \in \mathbb{N}$, and

$$
\left\|\left(D_{A}-1\right) \psi_{n}\right\|=\mathcal{O}\left(1 / R_{n}\right), \quad n \rightarrow \infty .
$$

(iv) $W$ fulfills Hypothesis 3 and, for every $\delta \in\left(0, \frac{1}{N}\right)$, we find some $\varepsilon \in(0,1)$ such that

$$
\limsup _{n \rightarrow \infty} R_{n}\left(v_{\star}\left(\delta, R_{n}\right)+(N-1) \sup _{|x-y| \geqslant(1-\varepsilon) R_{n}} W(x, y)\right)<0 .
$$

2.8 Example. $V=V_{\mathrm{C}}+V_{\mathrm{H}}+V_{\mathrm{E}}$ and $W$ fulfill Hypothesis 5 (i) and (iv), if $V_{\mathrm{C}}$ is given as in Example 2.1 with $\sum_{y \in \mathcal{Y}} Z_{y} \geqslant N, V_{\mathrm{H}}$ and $V_{\mathrm{E}}$ are given as in Example 2.2(i) or (ii), and $W$ is the Coulomb repulsion, $W(x, y)=e^{2} /|x-y|$.

Hypothesis 5(iii) is fulfilled under the following strengthened version of the condition given in [20]: Suppose again that $A \in C^{\infty}\left(\mathbb{R}^{3}, \mathbb{R}^{3}\right), B=\operatorname{curl} A$, and let $\mathcal{B}_{\rho_{n}}\left(z_{n}\right)$ denote the balls appearing in Example 2.6. Suppose additionally that there is some $C \in(0, \infty)$ such that $\rho_{n}<\left|z_{n}\right| \leqslant C \rho_{n}$, for all $n \in \mathbb{N}$, and that either

$$
\sup \left\{|B(x)|: x \in \mathcal{B}_{\rho_{n}}\left(z_{n}\right)\right\} \leqslant C /\left|z_{n}\right|^{2}, \quad n \in \mathbb{N},
$$

or

$$
\forall n \in \mathbb{N}:\left|B\left(z_{n}\right)\right| \geqslant 1 / C \text { and } \sup \left\{\epsilon_{\nu}(x) \mid x \in \mathcal{B}_{\rho_{n}}\left(z_{n}\right)\right\}=o\left(\rho_{n}^{-\nu}\right) .
$$

Then we find a Weyl sequence $\psi_{1}, \psi_{2}, \ldots$ satisfying the conditions in Hypothesis 5(iii). This follows by inspecting and adapting all relevant proofs in [20]. We leave this procedure to the reader since it is straightforward but a little bit lengthy.

2.9 Theorem. (Existence of bound states) Assume that $V, W$, and $A$ fulfill Hypothesis 5. Then $H_{N}^{\mathcal{A}}$ has infinitely many eigenvalues below the infimum of its essential spectrum, inf $\sigma_{\text {ess }}\left(H_{N}^{\mathcal{A}}\right)=\mathscr{E}_{N-1}^{\mathcal{A}}+1$.

Proof: This theorem is proved in Section 7. 


\section{Spectral projections of the Dirac operator}

In this section we study spectral projections of Dirac operators with singular potentials in magnetic fields. We start by recalling some basic well-known facts about Dirac operators in Subsection 3.1. A crucial role is played by the resolvent identity stated in that subsection which applies to Coulomb singularities corresponding to nuclear charges up to $Z \leqslant 137$. In Subsection 3.2 we derive some norm estimates on resolvents of Dirac operators which are conjugated with exponential weight functions. We verify that the decay rate is not smaller than the distance of the real part of the spectral parameter to the essential spectrum. The simple Neumann-type argument we employ to prove this for non-vanishing electrostatic potentials might be new. In Subsection 3.3 we derive the main technical tools of this paper, namely, various commutator estimates involving spectral projections of singular Dirac operators. Finally, in Subsection 3.4 we study the difference of projections with and without electrostatic potentials.

\subsection{Basic properties of Dirac operators with singular po- tentials in magnetic fields}

In the next lemma we collect various well-known results on Dirac operators which play an important role in the whole paper. To this end we let $H_{c}^{s}:=$ $H_{c}^{s}\left(\mathbb{R}^{3}, \mathbb{C}^{4}\right)$ denote all elements of $H^{s}:=H^{s}\left(\mathbb{R}^{3}, \mathbb{C}^{4}\right), s \in \mathbb{R}$, having compact support. Moreover, we denote the canonical extension of $D_{0}$ to an element of $\mathscr{L}\left(H^{1 / 2}, H^{-1 / 2}\right)$ by $\check{D}_{0}$. It shall sometimes be convenient to consider the singular part of $V_{\mathrm{C}}$,

$$
V_{\mathrm{C}}^{\mathrm{s}}(x):=\sum_{y \in \mathcal{Y}} \varrho(x-y) V_{\mathrm{C}}(x), \quad x \in \mathbb{R}^{3}
$$

where $\varrho \in C_{0}^{\infty}\left(\mathbb{R}^{3},[0,1]\right)$ equals 1 on $\mathcal{B}_{\varepsilon / 2}(0)$ and 0 outside $\mathcal{B}_{\varepsilon}(0)$. Here $\varepsilon$ is the parameter appearing in Hypothesis 1. We let $V_{\mathrm{C}}^{\mathrm{s}}(x)=S(x)\left|V_{\mathrm{C}}^{\mathrm{s}}\right|(x)$ denote the polar decomposition of $V_{\mathrm{C}}^{\mathrm{s}}(x)$. By Hardy's inequality we know that $V_{\mathrm{C}}^{\mathrm{s}}$ is a bounded operator from $H^{1}\left(\mathbb{R}^{3}, \mathbb{C}^{4}\right)$ to $L^{2}\left(\mathbb{R}^{3}, \mathbb{C}^{4}\right)$. By duality and interpolation it possesses a unique extension $\check{V}_{\mathrm{C}}^{\mathrm{s}} \in \mathscr{L}\left(H^{1 / 2}, H^{-1 / 2}\right)$. Given some $A \in L_{\mathrm{loc}}^{\infty}\left(\mathbb{R}^{3}, \mathbb{R}^{3}\right)$ we set $A^{\mathrm{s}}:=(1-\vartheta) A$, where $\vartheta \in C_{0}^{\infty}\left(\mathbb{R}^{3},[0,1]\right)$ is equal to 1 on some ball containing $\operatorname{supp}\left(V_{\mathrm{C}}^{\mathrm{s}}\right)$. We let $\alpha \cdot A^{\mathrm{s}}(x)=U(x)\left|\alpha \cdot A^{\mathrm{s}}(x)\right|$ denote the polar decomposition of $\alpha \cdot A^{\mathrm{s}}(x)$ and note that the operator sum $\check{D}_{0}+\alpha \cdot A^{\mathrm{s}}+\check{V}_{\mathrm{C}}^{\mathrm{s}}$ is well-defined as an element of $\mathscr{L}\left(H_{c}^{1 / 2}, H_{c}^{-1 / 2}\right)$, for every $A \in L_{\mathrm{loc}}^{\infty}\left(\mathbb{R}^{3}, \mathbb{R}^{3}\right)$. 
3.1 Lemma. ([8, 31, 32, 33]) Assume that $A \in L_{\mathrm{loc}}^{\infty}\left(\mathbb{R}^{3}, \mathbb{R}^{3}\right)$ and $V_{\mathrm{C}}$ fulfills Hypothesis 1. Then there is unique self-adjoint operator, $D_{A^{\mathrm{s}}, V_{\mathrm{C}}^{\mathrm{s}}}$, such that:

(i) $\mathcal{D}\left(D_{A^{\mathrm{s}}, V_{\mathrm{C}}^{\mathrm{s}}}\right) \subset H_{\mathrm{loc}}^{1 / 2}\left(\mathbb{R}^{3}, \mathbb{C}^{4}\right)$.

(ii) For all $\psi \in H_{c}^{1 / 2}\left(\mathbb{R}^{3}, \mathbb{C}^{4}\right)$ and $\phi \in \mathcal{D}\left(D_{A^{\mathrm{s}}, V_{\mathrm{C}}^{\mathrm{s}}}\right)$,

$$
\begin{aligned}
& \left\langle\psi \mid D_{A^{\mathrm{s}}, V_{\mathrm{C}}^{\mathrm{s}}} \phi\right\rangle=\left\langle\left.\left|D_{0}\right|^{1 / 2} \psi\left|\operatorname{sgn}\left(D_{0}\right)\right| D_{0}\right|^{1 / 2} \phi\right\rangle \\
& \quad+\left\langle\left.\left|\alpha \cdot A^{\mathrm{s}}\right|^{1 / 2} \psi|U| \alpha \cdot A^{\mathrm{s}}\right|^{1 / 2} \phi\right\rangle+\left\langle\left.\left|V_{\mathrm{C}}^{\mathrm{s}}\right|^{1 / 2} \psi|S| V_{\mathrm{C}}^{\mathrm{s}}\right|^{1 / 2} \phi\right\rangle .
\end{aligned}
$$

Notes: In [33, Proposition 4.3] it is observed that the claim follows from [8, Theorem 1.3] and [31, 32].

Consequently, we may define a self-adjoint operator,

$$
D_{A, V}:=D_{A^{\mathrm{s}}, V_{\mathrm{C}}^{\mathrm{s}}}+\alpha \cdot\left(A-A^{\mathrm{s}}\right)+\left(V_{\mathrm{C}}-V_{\mathrm{C}}^{\mathrm{s}}\right)+V_{\mathrm{H}}+V_{\mathrm{E}}
$$

on the domain $\mathcal{D}\left(D_{A, V}\right)=\mathcal{D}\left(D_{A^{\mathrm{s}}, V_{\mathrm{C}}^{\mathrm{s}}}\right)$. Notice that in (29) we only add bounded operators to $D_{A^{\mathrm{s}}, V_{\mathrm{C}}^{\mathrm{s}}}$. We state some of its properties in the following lemma where

$$
R_{A, V}(z):=\left(D_{A, V}-z\right)^{-1}, \quad z \in \varrho\left(D_{A, V}\right) .
$$

3.2 Lemma. ([8, 31, 32, 33]) Assume that $A \in L_{\text {loc }}^{\infty}\left(\mathbb{R}^{3}, \mathbb{R}^{3}\right)$ and that $V$ fulfills Hypothesis 2, Then the following assertions hold true:

(a) $\mathbb{1}_{\mathcal{B}_{R}(0)}\left(D_{A, V}-i\right)^{-1}$ is compact, for every $R>0$.

(b) $\sigma_{\mathrm{ess}}\left(D_{A, V}\right)=\sigma_{\mathrm{ess}}\left(D_{A}\right), \sigma\left(D_{A}\right) \subset(-\infty,-1] \cup[1, \infty)$.

(c) $D_{A, V}$ is essentially self-adjoint on

$$
\mathcal{D}_{e}:=\left\{\phi \in H_{c}^{1 / 2}\left(\mathbb{R}^{3}, \mathbb{C}^{4}\right): \check{D}_{0} \phi+\alpha \cdot A \phi+\check{V}_{\mathrm{C}}^{\mathrm{s}} \phi \in L^{2}\left(\mathbb{R}^{3}, \mathbb{C}^{4}\right)\right\}
$$

and, for $\phi \in \mathcal{D}_{e}, D_{A, V} \phi$ is given as a sum of four vectors in $H^{-1 / 2}$,

$$
D_{A, V} \phi=\check{D}_{0} \phi+\alpha \cdot A \phi+\check{V}_{\mathrm{C}}^{\mathrm{s}} \phi+\left(V-V_{\mathrm{C}}^{\mathrm{s}}\right) \phi .
$$

Moreover, $\mathcal{D}_{e}=\mathcal{D}\left(D_{A, V}\right) \cap \mathscr{E}^{\prime}$, where $\mathscr{E}^{\prime}$ denotes the dual space of $C^{\infty}\left(\mathbb{R}^{3}, \mathbb{C}^{4}\right)$.

(d) For $\chi \in C_{0}^{\infty}\left(\mathbb{R}^{3}\right)$ and $\phi \in \mathcal{D}\left(D_{A, V}\right)$, we have $\chi \phi \in \mathcal{D}_{e} \subset \mathcal{D}\left(D_{A, V}\right)$ and

$$
\left[D_{A, V}, \chi\right] \phi=-i(\alpha \cdot \nabla \chi) \phi+\left[V_{\mathrm{E}}, \chi\right] \phi \text {. }
$$

In particular, for $z \in \varrho\left(D_{A, V}\right)$,

$$
\begin{aligned}
{\left[\chi, R_{A, V}(z)\right] } & =R_{A, V}(z)\left[D_{A, V}, \chi\right] R_{A, V}(z) \\
& =R_{A, V}(z)\left(-i(\alpha \cdot \nabla \chi) \phi+\left[V_{\mathrm{E}}, \chi\right]\right) R_{A, V}(z) .
\end{aligned}
$$

(e) If $A$ is bounded, then $\mathcal{D}\left(D_{A, V}\right) \subset H^{1 / 2}\left(\mathbb{R}^{3}, \mathbb{C}^{4}\right)$. 
Notes: Since $V_{\mathrm{E}}$ is compact it is clear that all assertions hold true as soon as they hold for $V_{\mathrm{E}}=0$, which we assume in the following. To prove (a) we write

$$
\mathbb{1}_{\mathcal{B}_{R}(0)}\left(D_{A, V}-i\right)^{-1}=\left(\mathbb{1}_{\mathcal{B}_{R}(0)}\left|D_{0}\right|^{-1 / 2}\right)\left(\left|D_{0}\right|^{1 / 2} \chi\left(D_{A, V}-i\right)^{-1}\right),
$$

where $\chi \in C_{0}^{\infty}\left(\mathbb{R}^{3},[0,1]\right)$ equals 1 in a neighbourhood of $\mathcal{B}_{R}(0)$. Then we use that $\mathbb{1}_{\mathcal{B}_{R}(0)}\left|D_{0}\right|^{-1 / 2}$ is compact and that $\left|D_{0}\right|^{1 / 2} \chi\left(D_{A, V}-i\right)^{-1}$ is bounded by Lemma 3.1 and the closed graph theorem. By standard arguments we obtain the identity $\sigma_{\text {ess }}\left(D_{A, V}\right)=\sigma_{\text {ess }}\left(D_{A}\right)$ from (a) since $V$ drops off to zero at infinity; see, e.g., [36, §4.3.4]. The inclusion $\sigma\left(D_{A}\right) \subset(-\infty,-1] \cup[1, \infty)$ follows from supersymmetry arguments; see, e.g., [36, §5.6]. The assertions in (c) follow from [8, §2], (d) follows from [8, Lemma G], and (e) from [31].

Next, we recall the useful resolvent identity (33) (see, e.g., [15, 40]) which is used very often in the sequel. The vector potential $\widetilde{A}$ in (33) could for instance be the gradient of some gauge potential or just be equal to zero. We recall another well-known resolvent identity [31] in the beginning of Appendix A.

3.3 Lemma. Assume that $V$ fulfills Hypothesis 2, and that $A, \widetilde{A} \in L_{\mathrm{loc}}^{\infty}\left(\mathbb{R}^{3}, \mathbb{R}^{3}\right)$. Let $\widetilde{V}$ be either $V_{\mathrm{C}}^{\mathrm{s}}$ (given by $(28)$ ) or 0 , let $z \in \varrho\left(D_{\widetilde{A}, \widetilde{V}}\right) \cap \varrho\left(D_{A, V}\right)$ and $\chi \in C^{\infty}\left(\mathbb{R}^{3}, \mathbb{R}\right)$ be constant outside some ball in $\mathbb{R}^{3}$, and assume that $\left(V_{\mathrm{C}}-\widetilde{V}\right) \chi$ and $\alpha \cdot(A-\widetilde{A}) \chi$ are bounded. Then

$$
\begin{aligned}
\chi R_{A, V}(z)= & \chi R_{\widetilde{A}, \widetilde{V}}(z)+R_{\widetilde{A}, \widetilde{V}}(z) i \alpha \cdot(\nabla \chi)\left(R_{\widetilde{A}, \widetilde{V}}(z)-R_{A, V}(z)\right) \\
& -R_{\widetilde{A}, \widetilde{V}}(z) \chi(V-\widetilde{V}+\alpha \cdot(A-\widetilde{A})) R_{A, V}(z) .
\end{aligned}
$$

Proof: Let $\phi \in \widetilde{\mathcal{D}}_{e}:=\left\{\psi \in H_{c}^{1 / 2} \mid \check{D}_{0} \psi+\alpha \cdot \widetilde{A}+\widetilde{V} \psi \in L^{2}\right\}$. Since $\chi$ can be written as $\chi=c+\vartheta$, for some $c \in \mathbb{R}$ and $\vartheta \in C_{0}^{\infty}\left(\mathbb{R}^{3}, \mathbb{R}\right)$, Lemma 3.2(c)\&(d) imply that $\chi \phi \in \widetilde{\mathcal{D}}_{e}$. By the definition of $\mathcal{D}_{e}$ in (31) and the assumptions on $\chi$ it further follows that $\chi \phi \in \mathcal{D}_{e} \subset \mathcal{D}\left(D_{A, V}\right)$ and

$$
D_{\widetilde{A}, \widetilde{V}} \chi \phi=D_{A, V} \chi \phi+\{-V+\widetilde{V}-\alpha \cdot(A-\widetilde{A})\} \chi \phi .
$$

Therefore, we obtain

$$
\begin{aligned}
\left(R_{\widetilde{A}, \widetilde{V}}(z)-R_{A, V}(z)\right) \chi\left(D_{\widetilde{A}, \widetilde{V}}-z\right) \phi \\
=\quad\left(R_{\widetilde{A}, \widetilde{V}}(z)-R_{A, V}(z)\right)\left(\left(D_{\widetilde{A}, \widetilde{V}}-z\right) \chi+i \alpha \cdot(\nabla \chi)\right) \phi \\
=\quad \chi \phi-R_{A, V}(z)\left(D_{A, V}-z-V+\widetilde{V}-\alpha \cdot(A-\widetilde{A})\right) \chi \phi \\
\quad+\left(R_{\widetilde{A}, \widetilde{V}}(z)-R_{A, V}(z)\right) i \alpha \cdot(\nabla \chi) \phi \\
=\quad R_{A, V}(z)(V-\widetilde{V}+\alpha \cdot(A-\widetilde{A})) \chi R_{\widetilde{A}, \widetilde{V}}(z)\left(D_{\widetilde{A}, \widetilde{V}}-z\right) \phi \\
\quad+\left(R_{\widetilde{A}, \widetilde{V}}(z)-R_{A, V}(z)\right) i \alpha \cdot(\nabla \chi) R_{\widetilde{A}, \widetilde{V}}(z)\left(D_{\widetilde{A}, \widetilde{V}}-z\right) \phi .
\end{aligned}
$$


As $D_{\widetilde{A}, \widetilde{V}}$ is essentially self-adjoint on $\widetilde{\mathcal{D}}_{e}$, we know that $\left(D_{\widetilde{A}, \widetilde{V}}-z\right) \widetilde{\mathcal{D}}_{e}$ is dense, which together with the calculation above implies

$$
\begin{aligned}
\left(R_{\widetilde{A}, \widetilde{V}}(z)-R_{A, V}(z)\right) \chi= & \left(R_{\widetilde{A}, \widetilde{V}}(z)-R_{A, V}(z)\right) i \alpha \cdot(\nabla \chi) R_{\widetilde{A}, \widetilde{V}}(z) \\
& +R_{A, V}(z)(V-\widetilde{V}+\alpha \cdot(A-\widetilde{A})) \chi R_{\widetilde{A}, \widetilde{V}}(z) .
\end{aligned}
$$

Taking the adjoint of (34) (with $z$ replaced by $\bar{z}$ ) we obtain (33).

\subsection{Conjugation of $R_{A, V}(z)$ with exponential weights}

As a preparation for the localization estimates for the spectral projections we shall now study the conjugation of $R_{A, V}(z)$ with exponential weight functions $e^{F}$ acting as multiplication operators on $\mathscr{H}$. To this end we recall that $e_{0} \in$ $(-1,1)$ is an element of the resolvent set of $D_{A, V}$ and set

$$
\begin{aligned}
\delta_{0} & :=\inf \left\{\left|e_{0}-\lambda\right|: \lambda \in \sigma\left(D_{A, V}\right)\right\}>0, \\
\Gamma & :=e_{0}+i \mathbb{R} .
\end{aligned}
$$

Notice that the decay rate in the following lemma is determined only by the decay rate $m$ appearing in Hypothesis 2 and the number $\triangle_{0}$ defined in (16). In the next proof and henceforth we shall often use the abbreviations

$$
D_{A}:=D_{A, 0}, \quad R_{A}(z):=\left(D_{A}-z\right)^{-1}, \quad z \in \varrho\left(D_{A}\right) .
$$

3.4 Lemma. Assume that $A \in L_{\mathrm{loc}}^{\infty}\left(\mathbb{R}^{3}, \mathbb{R}^{3}\right)$ and that $V$ fulfills Hypothesis 2 . Let $0<a<\min \left\{\triangle_{0}, m\right\}$. Then there is some $C_{a} \in(0, \infty)$ such that, for all $F \in C^{\infty}\left(\mathbb{R}^{3}, \mathbb{R}\right)$ with $F(0)=0, F \geqslant 0$ or $F \leqslant 0,\|\nabla F\|_{\infty} \leqslant a$, and all $z=e_{0}+i \eta \in \Gamma$,

$$
\left\|e^{F} R_{A, V}(z) e^{-F}\right\| \leqslant \frac{C_{a}}{\sqrt{1+\eta^{2}}} .
$$

Proof: First, we assume that $F$ is constant outside some ball in $\mathbb{R}^{3}$. Then it suffices to treat the case $F \geqslant 0$, since otherwise we could consider the adjoint of $e^{F} R_{A, V}(z) e^{-F}$. To begin with we recall the identity

$$
\|\alpha \cdot v\|_{\mathscr{L}\left(\mathbb{C}^{4}\right)}=|v|, \quad v \in \mathbb{R}^{3},
$$

which follows for instance from the $C^{*}$-equality and the Clifford algebra relations (6). In particular,

$$
\|\alpha \cdot \nabla F\|\left\|R_{A}(z)\right\| \leqslant \frac{a}{\sqrt{\triangle_{0}^{2}+\eta^{2}}}<1, \quad z=e_{0}+i \eta \in \Gamma .
$$


Since $F$ is smooth and constant outside some compact set Lemma 3.2(d) permits to get, for $z \in \Gamma$,

$$
e^{F} R_{A}(z) e^{-F}=R_{A}(z) \sum_{\ell=0}^{\infty}\left\{-i(\alpha \cdot \nabla F) R_{A}(z)\right\}^{\ell},
$$

whence

$$
\left\|e^{F} R_{A}(z) e^{-F}\right\| \leqslant \frac{1}{1-a / \triangle_{0}} \frac{1}{\sqrt{\triangle_{0}^{2}+\eta^{2}}}, \quad z=e_{0}+i \eta \in \Gamma .
$$

Next, we pick some $R>\max \{|y|: y \in \mathcal{Y}\}$ and $\chi \in C^{\infty}\left(\mathbb{R}^{3},[0,1]\right)$ such that $\chi \equiv 0$ on $\mathcal{B}_{R}(0), \chi \equiv 1$ on $\mathbb{R}^{3} \backslash \mathcal{B}_{R+2}(0)$, and $\|\nabla \chi\|_{\infty} \leqslant 1$. We set $\bar{\chi}:=1-\chi$. Furthermore, we let $\tilde{\chi}$ denote the characteristic function of $\mathbb{R}^{3} \backslash \mathcal{B}_{R}(0)$. We choose $R$ so large (depending on $a$, but not on $F$; recall (13) ) that

$$
\sup _{|x| \geqslant R}\left\|V_{\mathrm{C}}(x)\right\|+\sup _{|x| \geqslant R}\left\|V_{\mathrm{H}}(x)\right\|+\left\|\tilde{\chi} e^{F} V_{\mathrm{E}} e^{-F} \tilde{\chi}\right\| \leqslant \frac{\triangle_{0}}{2}\left(1-\frac{a}{\triangle_{0}}\right) .
$$

Conjugating (33) with exponential weights and rearranging the terms we find, for $z \in \Gamma$,

$$
\begin{aligned}
\{\mathbb{1}+ & \left.e^{F} R_{A}(z) e^{-F}\left(\tilde{\chi} V_{\mathrm{C}}+\tilde{\chi} V_{\mathrm{H}}+\chi e^{F} V_{\mathrm{E}} e^{-F} \tilde{\chi}\right)\right\} \chi e^{F} R_{A, V}(z) e^{-F} \\
= & \chi e^{F} R_{A}(z) e^{-F}-\left(e^{F} R_{A}(z) e^{-F}\right)\left(e^{F} i \alpha \cdot \nabla \chi\right)\left(R_{A, V}(z)-R_{A}(z)\right) e^{-F} \\
& -\left(e^{F} R_{A}(z) e^{-F}\right)\left(\chi e^{F} V_{\mathrm{E}} e^{-F} \bar{\chi}\right)\left(\mathbb{1}_{\mathcal{B}_{R+2}(0)} e^{F}\right) R_{A, V}(z) e^{-F} .
\end{aligned}
$$

Here the operator $\{\cdots\}$ on the left side can be inverted by means of a Neumann series and $\left\|\{\cdots\}^{-1}\right\| \leqslant 2$ by (41) \&(42). Furthermore, we observe that, by the choice of $\chi$, the assumption on $F$, and (39),

$$
\left\|e^{F} i \alpha \cdot \nabla \chi\right\| \leqslant e^{a(R+2)}, \quad\left\|R_{A}(z)\right\| \leqslant 1, \quad\left\|R_{A, V}(z)\right\| \leqslant \frac{1}{\delta_{0}}, \quad\left\|e^{-F}\right\| \leqslant 1 .
$$

Using these remarks together with (12) and (41) we obtain

$$
\left\|\chi e^{F} R_{A, V}(z) e^{-F}\right\| \leqslant \frac{C_{a}^{\prime} e^{R+2}}{\sqrt{\triangle_{0}^{2}+\eta^{2}}} .
$$

This estimate implies the assertion if $F$ is constant outside some ball since, certainly, $\left\|\bar{\chi} e^{F}\right\| \leqslant e^{a(R+2)}$ and, for $z=e_{0}+i \eta \in \Gamma$,

$$
\left\|R_{A, V}(z)\right\| \leqslant \frac{1}{\sqrt{\delta_{0}^{2}+\eta^{2}}} .
$$


Let us now assume that $F \geqslant 0$ is not necessarily bounded. Let $F_{1}, F_{2}, \cdots \in$ $C^{\infty}\left(\mathbb{R}^{3},[0, \infty)\right)$ be constant outside some ball and such that $F_{n}=F$ on $\mathcal{B}_{n}(0)$ and $F_{n} \rightarrow F$. Then $e^{-F_{n}} R_{A, V}(z) e^{F_{n}} \phi \rightarrow e^{-F} R_{A, V}(z) e^{F} \phi$ by the dominated convergence theorem, for every $\phi \in \mathscr{D}$. Since $e^{-F_{n}} R_{A, V}(z) e^{F_{n}}$ obeys the estimate (38) uniformly in $n$, we see that the densely defined operator $e^{-F} R_{A, V}(z) e^{F} \Gamma_{\mathscr{D}}$ is bounded and satisfies (38), too. But this is the case if and only if its adjoint, $e^{F} R_{A, V}(z) e^{-F}=\left(e^{-F} R_{A, V}(z) e^{F}\right)^{*}$, is an element of $\mathscr{L}(\mathscr{H})$ and satisfies (38) as well.

In the applications of the previous lemma the following observation is very useful.

3.5 Lemma. Assume that $A \in L_{\text {loc }}^{\infty}\left(\mathbb{R}^{3}, \mathbb{R}^{3}\right)$ and that $V$ fulfills Hypothesis 2 , Let $0<a<\min \left\{\triangle_{0}, m\right\}$. Then there is some $C_{a}^{\prime} \in(0, \infty)$ such that, for all $F \in C^{\infty}\left(\mathbb{R}^{3}, \mathbb{R}\right)$ with $F(0)=0, F \geqslant 0$ or $F \leqslant 0,\|\nabla F\|_{\infty} \leqslant a$, which are constant outside some ball in $\mathbb{R}^{3}$, and for all $\phi \in \mathscr{H}$,

$$
\int_{\Gamma}\left\|\left|D_{A, V}\right|^{1 / 2} e^{F} R_{A, V}(z) e^{-F} \phi\right\|^{2}|d z| \leqslant C_{a}^{\prime}\|\phi\|^{2}
$$

and, for $\phi \in \mathcal{D}\left(\left|D_{A, V}\right|^{1 / 2}\right)$,

$$
\int_{\Gamma}\left\|e^{F} R_{A, V}(z) e^{-F}\left|D_{A, V}\right|^{1 / 2} \phi\right\|^{2}|d z| \leqslant C_{a}^{\prime}\|\phi\|^{2} .
$$

Proof: For later reference we additionally pick some $\chi \in C^{\infty}\left(\mathbb{R}^{3}, \mathbb{R}\right)$ which is constant outside some large ball and infer from Lemma 3.2(e) that, for $z \in \Gamma$,

$$
\begin{aligned}
{\left[R_{A, V}(z), \chi e^{F}\right]=} & R_{A, V}(z)\{i \alpha \cdot(\nabla \chi+\chi \nabla F) \\
& \left.+\left[\chi e^{F}, V_{\mathrm{E}}\right] e^{-F}\right\} e^{F} R_{A, V}(z) .
\end{aligned}
$$

The special case $\chi \equiv 1$ implies

$$
\begin{aligned}
e^{F} R_{A, V}(z) e^{-F}= & R_{A, V}(z)-R_{A, V}(z)\{i \alpha \cdot \nabla F \\
& \left.+\left[e^{F}, V_{\mathrm{E}}\right] e^{-F}\right\} e^{F} R_{A, V}(z) e^{-F} .
\end{aligned}
$$

Taking the adjoint and replacing $F$ by $-F$ and $\bar{z}$ by $z$ we also get

$$
\begin{aligned}
e^{F} R_{A, V}(z) e^{-F}= & R_{A, V}(z)-e^{F} R_{A, V}(z) e^{-F}\{i \alpha \cdot \nabla F \\
& \left.+e^{F}\left[V_{\mathrm{E}}, e^{-F}\right]\right\} R_{A, V}(z) .
\end{aligned}
$$

Now, let $T$ be a self-adjoint operator on some Hilbert space $\mathscr{K}$ such that $\left(-\delta_{0}, \delta_{0}\right) \subset \varrho(T)$. Then, for $\phi \in \mathscr{K}$,

$$
\int_{\mathbb{R}}\left\||T|^{1 / 2}(T-i \eta)^{-1} \phi\right\|^{2} d \eta=\int_{\mathbb{R}} \int_{\mathbb{R}} \frac{|\lambda|}{\lambda^{2}+\eta^{2}} d \eta d\left\|E_{\lambda}(T) \phi\right\|^{2}=\pi\|\phi\|^{2},
$$


and it is elementary to check that, for $\eta \in \mathbb{R}$,

$$
\left\||T|^{1 / 2}(T-i \eta)^{-1}\right\|=\frac{\delta_{0}^{1 / 2} \mathbb{1}_{\left(-\delta_{0}, \delta_{0}\right)}(\eta)}{\sqrt{\delta_{0}^{2}+\eta^{2}}}+\frac{\mathbb{1}_{\left(-\delta_{0}, \delta_{0}\right)^{c}}(\eta)}{\sqrt{2|\eta|}}
$$

Using (49) and (150) with $T=D_{A, V}-e_{0}$ and taking (13), (38), (39), and (43) into account we readily derive the asserted estimate (44) from (47). The second estimate (45) it obtained analogously by means of (48).

\subsection{Commutators}

In this subsection we derive the crucial technical prerequisits for the spectral analysis of $H_{N}$, namely various commutator estimates involving the projection $\Lambda_{A, V}^{+}$, cut-off functions, and exponential weights $e^{F}$. Our standard assumptions on the cut-off and weight functions are

$$
\chi \in C^{\infty}\left(\mathbb{R}^{3},[0,1]\right) \text { is constant outside some ball. }
$$

and

$$
\left.\begin{array}{l}
F \in C^{\infty}\left(\mathbb{R}^{3}, \mathbb{R}\right), \quad F \geqslant 0 \text { or } F \leqslant 0, \quad F(0)=0, \quad|\nabla F| \leqslant a, \\
F \text { is constant outside some ball. }
\end{array}\right\}
$$

To shorten the presentation we generalize our estimates to unbounded $F$ only if this is explicitely used in this article.

3.6 Proposition. Assume that $A \in L_{\text {loc }}^{\infty}\left(\mathbb{R}^{3}, \mathbb{R}^{3}\right)$ and that $V$ fulfills Hypothesis 2 and let $0<a_{0}<\min \left\{\triangle_{0}, m\right\}$. Then there is some constant $C_{a_{0}} \in(0, \infty)$ such that, for all $a \in\left[0, a_{0}\right]$ and $\chi, F$ satisfying (51) and (52),

$$
\left\|\left|D_{A, V}\right|^{1 / 2}\left[\Lambda_{A, V}^{+}, \chi e^{F}\right] e^{-F}\left|D_{A, V}\right|^{1 / 2}\right\|=C_{a_{0}}\left(\|\nabla \chi\|_{\infty}+a\right) .
$$

Proof: We shall employ the identity

$$
\left[\Lambda_{A, V}^{+}, \chi e^{F}\right]=\frac{1}{2}\left[\operatorname{sgn}\left(D_{A, V}-e_{0}\right), \chi e^{F}\right]
$$

and the representation of the sign function as a Cauchy principal value (see, e.g., [24, Page 359]),

$$
\operatorname{sgn}\left(D_{A, V}-e_{0}\right) \psi=\int_{\Gamma} R_{A, V}(z) \psi \frac{d z}{\pi}:=\lim _{R \rightarrow \infty} \int_{-R}^{R} R_{A, V}\left(e_{0}+i \eta\right) \psi \frac{d \eta}{\pi},
$$


for $\psi \in \mathscr{H}$, where $\Gamma$ is defined in (36). Taking also (11), (12), and (46) in to account we obtain

$$
\begin{aligned}
& \left|\left\langle\left.\left|D_{A, V}\right|^{1 / 2} \phi\left|\left[\Lambda_{A, V}^{+}, \chi e^{F}\right] e^{-F}\right| D_{A, V}\right|^{1 / 2} \psi\right\rangle\right| \\
& \leqslant \int_{\Gamma}\left\|\left|D_{A, V}\right|^{1 / 2} R_{A, V}(\bar{z}) \phi\right\|\left\|i \alpha \cdot(\nabla \chi+\chi \nabla F)+\left[\chi e^{F}, V_{\mathrm{E}}\right] e^{-F}\right\| \\
& \cdot\left\|e^{F} R_{A, V}(z) e^{-F}\left|D_{A, V}\right|^{1 / 2} \psi\right\| \frac{|d z|}{2 \pi} \\
& \leqslant C_{a_{0}}^{\prime}\left(\|\nabla \chi\|_{\infty}+\|\chi \nabla F\|_{\infty}+a\right)\left(\int_{\Gamma}\left\|\left|D_{A, V}\right|^{1 / 2} R_{A, V}(z) \phi\right\|^{2} \frac{|d z|}{2 \pi}\right)^{1 / 2} \\
& \cdot\left(\int_{\Gamma}\left\|e^{F} R_{A, V}(z) e^{-F}\left|D_{A, V}\right|^{1 / 2} \psi\right\|^{2} \frac{|d z|}{2 \pi}\right)^{1 / 2}
\end{aligned}
$$

for $\phi, \psi \in \mathcal{D}\left(\left|D_{A}\right|^{1 / 2}\right) \supset \operatorname{Ran}\left(R_{A}(z)\right)$. By virtue of (44) \&(45) we first infer that

$$
\left[\Lambda_{A, V}^{+}, \chi e^{F}\right] e^{-F}\left|D_{A, V}\right|^{1 / 2} \psi \in \mathcal{D}\left(\left(\left|D_{A, V}\right|^{1 / 2}\right)^{*}\right)=\mathcal{D}\left(\left|D_{A, V}\right|^{1 / 2}\right) .
$$

We conclude by recalling that an operator $T: \mathcal{D}(T) \rightarrow \mathscr{K}$ on some Hilbert space $\mathscr{K}$ is bounded if and only if

$$
\sup \{|\langle\phi \mid T \psi\rangle|: \phi \in X, \psi \in \mathcal{D}(T),\|\phi\|=\|\psi\|=1\}
$$

is finite, in which case it is equal to the norm of $T$. Here $X \subset \mathscr{K}$ is a subspace with $\bar{X} \supset \operatorname{Ran}(T)$.

Given some suitable weight function, $F$, we abbreviate

$$
\Lambda_{A, V}^{F}:=e^{F} \Lambda_{A, V}^{+} e^{-F} .
$$

3.7 Corollary. Assume that $A \in L_{\mathrm{loc}}^{\infty}\left(\mathbb{R}^{3}, \mathbb{R}^{3}\right)$ and that $V$ fulfills Hypothesis 2 . Let $0<a<\min \left\{\triangle_{0}, m\right\}$. Then there is some $C(a) \in(0, \infty)$ such that, for all $F \in C^{\infty}\left(\mathbb{R}^{3}, \mathbb{R}\right)$ satisfying $F(0)=0, F \geqslant 0$ or $F \leqslant 0$, and $\|\nabla F\|_{\infty} \leqslant a$, we have $\Lambda_{A, V}^{F} \in \mathscr{L}(\mathscr{H})$ and $\left\|\Lambda_{A, V}^{F}\right\| \leqslant C(a)$.

Proof: First, we assume that $F$ satisfies (52). In this case the claim follows from Proposition 3.6 because $\left[e^{F}, \Lambda_{A, V}^{+}\right] e^{-F}=\Lambda_{A, V}^{F}-\Lambda_{A, V}^{+}$. If $F$ is unbounded, then we apply an approximation argument similar to the one at the end of the proof of Lemma 3.4.

3.8 Corollary. Assumme that $A \in L_{\mathrm{loc}}^{\infty}\left(\mathbb{R}^{3}, \mathbb{R}^{3}\right)$ and that $V$ fulfills Hypothesis 2 and let $0<a_{0}<\min \left\{\triangle_{0}, m\right\}$. Then there is some constant $C \in(0, \infty)$ 
such that, for all $a \in\left[0, a_{0}\right], \chi, F$ satisfying (51),(152), and $\|\nabla \chi\|_{\infty} \leqslant 1$, $L \in \mathscr{L}(\mathscr{H})$, and $\varphi \in \mathscr{H}$,

$$
\begin{aligned}
\mid\left\langle\varphi \mid \Lambda_{A, V}^{F} \chi L \chi \Lambda_{A, V}^{F} \varphi\right\rangle & -\left\langle\varphi \mid \chi \Lambda_{A, V}^{+} L \Lambda_{A, V}^{+} \chi \varphi\right\rangle \mid \\
& \leqslant\left(a+\|\nabla \chi\|_{\infty}\right) C\|L\|\|\varphi\|^{2} .
\end{aligned}
$$

Moreover, for all $\varphi \in \mathcal{D}\left(D_{A, V}\right)$,

$$
\begin{aligned}
& \left|\left\langle\varphi \mid \Lambda_{A, V}^{F} \chi D_{A, V_{\mathrm{C}}} \chi \Lambda_{A, V}^{F} \varphi\right\rangle-\left\langle\varphi \mid \chi \Lambda_{A, V}^{+} D_{A, V_{\mathrm{C}}} \Lambda_{A, V}^{+} \chi \varphi\right\rangle\right| \\
& \quad \leqslant\left(a+\|\nabla \chi\|_{\infty}\right) \inf _{0<\varepsilon \leqslant 1}\left\{\varepsilon\left\langle\varphi \mid \chi \Lambda_{A, V}^{+} D_{A, V_{\mathrm{C}}} \Lambda_{A, V}^{+} \chi \varphi\right\rangle+C \varepsilon^{-1}\|\varphi\|^{2}\right\} .
\end{aligned}
$$

If $V_{\mathrm{C}}=V=0$, then (60) still holds true, if $\chi D_{A} \chi$ is replaced by $\chi\left|D_{A}\right| \chi$ on the left side.

Proof: The proof of (59) is a rather obvious application of Proposition 3.6 and in fact a simpler analogue of the derivation of (60) below. Once (59) is verified, it suffices to prove (60) with $D_{A, V_{\mathrm{C}}}$ replaced by $D_{A, V}$ since $V_{\mathrm{H}}$ and $V_{\mathrm{E}}$ are bounded. Without loss of generality we may further assume that $D_{A, V}$ is positive on the range of $\Lambda_{A, V}^{+}$. For otherwise we could add a suitable constant to $D_{A, V}$. To prove (60) we first recall that $\Lambda_{A, V}^{+}$maps the domain of $D_{A, V}$ into itself and by Lemma 3.2(d) we know that multiplication with $\chi$ or $e^{ \pm F}$ leaves $\mathcal{D}\left(D_{A, V}\right)$ invariant, too. We thus have the following identity on $\mathcal{D}\left(D_{A, V}\right)$,

$$
\begin{aligned}
& \Lambda_{A, V}^{F} \chi D_{A, V} \chi \Lambda_{A, V}^{F}-\chi \Lambda_{A, V}^{+} D_{A, V} \chi \\
& =e^{F}\left[\Lambda_{A, V}^{+}, \chi e^{-F}\right] D_{A, V} \Lambda_{A, V}^{+} \chi+\chi \Lambda_{A, V}^{+} D_{A, V}\left[\chi e^{F}, \Lambda_{A, V}^{+}\right] e^{-F} \\
& \quad+e^{F}\left[\Lambda_{A, V}^{+}, \chi e^{-F}\right] D_{A, V}\left[\chi e^{F}, \Lambda_{A, V}^{+}\right] e^{-F} .
\end{aligned}
$$

It follows that the absolute value on the left side of (60) is less than or equal to

$$
\begin{aligned}
\|\left|D_{A, V}\right|^{1 / 2} \Lambda_{A, V}^{+} \chi \varphi & \left\|\left\{\sum_{\sharp= \pm}\left\|\left|D_{A, V}\right|^{1 / 2}\left[\Lambda_{A, V}^{+}, \chi e^{\sharp F}\right] e^{-\sharp F}\right\|\right\}\right\| \varphi \| \\
& +\prod_{\sharp= \pm}\left\|\left|D_{A, V}\right|^{1 / 2}\left[\Lambda_{A, V}^{+}, \chi e^{\sharp F}\right] e^{-\sharp F}\right\|\|\varphi\|^{2},
\end{aligned}
$$

which together with Proposition 3.6 implies (60) $)$. The last statement of the lemma is valid since the argument above works equally well with $\left|D_{A, V}\right|$ in place of $D_{A, V}$ because $\Lambda_{A, V}^{+}\left|D_{A, V}\right|=\Lambda_{A, V}^{+} D_{A, V}$.

3.9 Lemma. Assume that $A \in L_{\mathrm{loc}}^{\infty}\left(\mathbb{R}^{3}, \mathbb{R}^{3}\right)$ satisfies $\left\|A e^{-\tau_{0}|x|}\right\|_{\infty}<\infty$, for some $0 \leqslant \tau_{0}<\min \left\{m, \triangle_{0}\right\}$, and that $V$ fulfills Hypothesis 2 . Then $\Lambda_{A, V}^{+} \phi \in$ $H^{1 / 2}\left(\mathbb{R}^{3}, \mathbb{C}^{4}\right)$, for every $\phi \in \mathscr{D}$. 
Proof: Let $\phi \in \mathscr{D}$. We pick some $\chi \in C_{0}^{\infty}\left(\mathbb{R}^{3},[0,1]\right)$ with $\chi \equiv 1$ on $\operatorname{supp}(\phi)$. Furthermore, we pick $\zeta \in C_{0}^{\infty}\left(\mathbb{R}^{3},[0,1]\right)$ such that $\zeta \equiv 1$ on $\operatorname{supp}(\chi) \cup \mathcal{B}_{R}(0)$, where $R>\max \{|y|: y \in \mathcal{Y}\}$. We set $\bar{\zeta}:=1-\zeta$. Since $\mathcal{D}\left(D_{A, V}\right) \subset H_{\text {loc }}^{1 / 2}\left(\mathbb{R}^{3}, \mathbb{C}^{4}\right)$ and the spectral projection $\Lambda_{A, V}^{+}$maps the domain of $D_{A, V}$ into itself it follows that $\zeta \Lambda_{A, V}^{+} \phi \in H^{1 / 2}\left(\mathbb{R}^{3}, \mathbb{C}^{4}\right)$. Furthermore, we pick a (smooth, locally finite) partition of unity on $\mathbb{R}^{3},\left\{J_{\nu}\right\}_{\nu \in \mathbb{N}}, \sum_{\nu=1}^{\infty} J_{\nu}=1$, such that $\sum_{\nu=1}^{\infty}\left|\nabla J_{\nu}\right| \leqslant C$, for some constant $C \in(0, \infty)$. Setting $\zeta_{\nu}:=J_{\nu} \bar{\zeta}, \nu \in \mathbb{N}, \widetilde{\phi}:=\Lambda_{A, V}^{+}\left(D_{A, V}-i\right) \phi$, and using (33), we obtain

$$
\begin{aligned}
\bar{\zeta} \Lambda_{A, V}^{+} \phi= & \sum_{\nu=1}^{\infty} \zeta_{\nu} R_{A, V}(i) \Lambda_{A, V}^{+}\left(D_{A, V}-i\right) \phi \\
= & \bar{\zeta} R_{0}(i) \widetilde{\phi}-\sum_{\nu=1}^{\infty} R_{0}(i) i \alpha \cdot\left(\nabla \zeta_{\nu}\right)\left(R_{A, V}(i)-R_{0}(i)\right) \widetilde{\phi} \\
& -\sum_{\nu=1}^{\infty} R_{0}(i) \zeta_{\nu}(V+\alpha \cdot A) R_{A, V}(i) \tilde{\phi}
\end{aligned}
$$

Here the sum in (61) commutes with the first resolvent and the strong limit $\sum_{\nu=1}^{\infty} i \alpha \cdot\left(\nabla \zeta_{\nu}\right)$ defines a bounded operator on $L^{2}\left(\mathbb{R}^{3}, \mathbb{C}^{4}\right)$. To treat (62) we first use that $\sum_{\nu=1}^{\infty} \zeta_{\nu} V=\bar{\zeta} V$ is bounded. Next, we pick some $F \in C^{\infty}\left(\mathbb{R}^{3},[0, \infty)\right)$ vanishing on some ball containing 0 and $\operatorname{supp}(\phi)$ and satisfying $F(x)=a|x|-a^{\prime}$, for $x$ outside some sufficiently large ball with $\tau_{0}<a<\min \left\{m, \triangle_{0}\right\}, a^{\prime}>0$. Then we write

$$
\begin{aligned}
(\alpha \cdot A) & R_{A, V}(i) \widetilde{\phi}=\left(e^{-F} \alpha \cdot A\right)\left(e^{F} R_{A, V}(i) e^{-F}\right) \times \\
& \times\left(e^{F} \Lambda_{A, V}^{+} e^{-F}\right)\left(D_{A, V_{\mathrm{C}}+V_{\mathrm{H}}}+i \alpha \cdot \nabla F+e^{F} V_{\mathrm{E}} e^{-F}\right) \phi .
\end{aligned}
$$

Using (12), Lemma 3.4, and Corollary 3.7 we see that $(\alpha \cdot A) R_{A, V}(i) \widetilde{\phi}$ is an element of $L^{2}\left(\mathbb{R}^{3}, \mathbb{C}^{4}\right)$. These remarks imply that $\bar{\zeta} \Lambda_{A, V}^{+} \phi$ belongs to $\operatorname{Ran}\left(R_{0}(i)\right)+$ $\operatorname{Ran}\left(\bar{\zeta} R_{0}(i)\right)=H^{1}\left(\mathbb{R}^{3}, \mathbb{C}^{4}\right)$.

We may now conclude that $H_{N}$ is well-defined on the dense domain $\mathscr{D}_{N}$ defined in (18).

3.10 Corollary. Assume that $A \in L_{\text {loc }}^{\infty}\left(\mathbb{R}^{3}, \mathbb{R}^{3}\right)$ satisfies $\left\|A e^{-\tau_{0}|x|}\right\|_{\infty}<\infty$, for some $0 \leqslant \tau_{0}<\min \left\{m, \triangle_{0}\right\}$, and that $V$ fulfills Hypothesis 2, Then, for $\Psi \in \mathscr{D}_{N}$ and $1 \leqslant i<j \leqslant N$,

$$
\int_{\mathbb{R}^{3 N}} \frac{1}{\left|x_{i}-x_{j}\right|^{2}}\left|\Lambda_{A, V}^{+, N} \Psi(X)\right|^{2} d X<\infty .
$$


Proof: Let $\phi, \psi \in \mathscr{D}$. Thanks to Lemma 3.9 we know that both $\Lambda_{A, V}^{+} \phi$ and $\Lambda_{A, V}^{+} \psi$ belong to $H^{1 / 2}\left(\mathbb{R}^{3}, \mathbb{C}^{4}\right)$ and, hence, to $L^{3}\left(\mathbb{R}^{3}, \mathbb{C}^{4}\right)$ by the Sobolev inequality for $\left|\frac{1}{i} \nabla\right|$. An application of the Hardy-Littlewood-Sobolev inequality thus yields

$$
\int_{\mathbb{R}^{6}} \frac{1}{|x-y|^{2}}\left|\Lambda_{A, V}^{+} \phi(x)\right|^{2}\left|\Lambda_{A, V}^{+} \psi(y)\right|^{2} d x d y<\infty .
$$

This estimate clearly implies the full assertion.

In our applications it is important to control commutators that are multiplied with square-roots of the electron-electron interactions $W\left(x_{i}, x_{j}\right)$. In order to formulate an appropriate estimate we set

$$
W_{y}(x):=W(x, y)=W(y, x), \quad x, y \in \mathbb{R}^{3},
$$

in what follows. The proof of the next proposition looks somewhat lengthy and is hence postponed to Appendix A. This is due to the fact that the singularity of $W_{y}$ may be located anywhere and that we allow for unbounded magnetic fields. We remark that, even in the case $V=0$, a diamagnetic inequality is not very useful in this context since, for unbounded magnetic fields, one cannot compare $|-i \nabla+A|$ with $\left|D_{A}\right|$. We tackle this problem by a procedure that involves a partition of unity, local gauge transformations, and exponential decay estimates which control the correlation between different regions in position space. As a result we obtain a commutator estimate which can be chosen to depend only on the local magnitude of $|B|$ either at the singularity $y$ or on the support of the involved cut-off function. For any function $\chi$ on $\mathbb{R}^{3}$ we use the notation

$$
\|B\|_{\infty, \chi}:=\sup \{|B(x)|: x \in \operatorname{supp}(\chi)\} \text {. }
$$

3.11 Proposition. Assume that $A \in C^{1}\left(\mathbb{R}^{3}, \mathbb{R}^{3}\right)$ and $B=\operatorname{curl} A$ satisfies (23) and that $V$ fulfills Hypothesis 2. Let $0 \leqslant a_{0}<\min \left\{m, \triangle_{0}\right\}$ and $\mathcal{N} \subset \mathbb{R}^{3}$ be a neighbourhood of the set of singularities, $\mathcal{Y}$, of $V_{\mathrm{C}}$. Then there is some constant, $C_{a_{0}, \mathcal{N}} \in(0, \infty)$, such that, for all $a \in\left[0, a_{0}\right]$, all $\chi, F$ satisfying (51),(52) which are constant on $\mathcal{N}$, and all $y \in \mathbb{R}^{3}$,

$$
\begin{aligned}
& \left\|W_{y}^{1 / 2}\left[\Lambda_{A, V}^{+}, e^{F} \chi\right] e^{-F}\right\| \\
& \quad \leqslant C_{a_{0}, \mathcal{N}}\left(1+\min \left\{|B(y)|,\|B\|_{\infty, \chi}\right\}\right)\left(a+\|\nabla \chi\|_{\infty}\right) .
\end{aligned}
$$

If $V_{\mathrm{E}}=0$, then $\|B\|_{\infty, \chi}$ can be replaced by $\|B\|_{\infty, \chi \nabla F+\nabla \chi}$ in (65). 
3.12 Corollary. Assume that $A \in C^{1}\left(\mathbb{R}^{3}, \mathbb{R}^{3}\right)$ and $B=\operatorname{curl} A$ is bounded and that $V$ fulfills Hypothesis 2. Then we find, for every $\varepsilon>0$, some constant $C_{a_{0}, \varepsilon} \in(0, \infty)$ such that, for all $F$ satisfying (52), $\varphi \in \mathscr{D}_{N}$, and $1 \leqslant i \leqslant N$,

$$
\begin{aligned}
& \left|\left\langle\varphi \mid \mathbb{1} \otimes e^{F} \Lambda_{A, V}^{+, N} W_{i N} \Lambda_{A, V}^{+, N} \mathbb{1} \otimes e^{-F} \varphi\right\rangle-\left\langle\varphi \mid \Lambda_{A, V}^{+, N} W_{i N} \Lambda_{A, V}^{+, N} \varphi\right\rangle\right| \\
& \quad \leqslant a\left\{\varepsilon\left\langle\varphi \mid \Lambda_{A, V}^{+, N} W_{i N} \Lambda_{A, V}^{+, N} \varphi\right\rangle+C_{a_{0}, \varepsilon}\|\varphi\|^{2}\right\},
\end{aligned}
$$

where $e^{F}$ acts only on the last variable.

Proof: This corollary is proved by means of Proposition 3.11 in the same way as Corollary 3.8. We also recall that $\Lambda_{A, V}^{+, N} \mathscr{D}_{N} \subset \mathcal{D}\left(W_{i j}\right)$.

The technique used in the proof of Proposition 3.11 also yields the following result whose proof can be found in Appendix $\mathrm{A}$, too:

3.13 Lemma. Assume that $A \in C^{1}\left(\mathbb{R}^{3}, \mathbb{R}^{3}\right)$ and $B=\operatorname{curl} A$ satisfies (23) and that $V$ fulfills Hypothesis 2. Then there is some constant $C \in(0, \infty)$ such that, for all $\psi \in \mathcal{D}\left(D_{A, V}\right)$,

$$
\left\|W_{y}^{1 / 2} \Lambda_{A, V}^{+} \psi\right\| \leqslant C\left(1+\min \left\{|B(y)|,\|B\|_{\infty, \psi}\right\}\right)\left\|\left(D_{A, V}-i\right) \psi\right\|
$$

\subsection{Differences of projections}

In our applications it is eventually necessary to have some control on the difference between $\Lambda_{A, V}^{+}$and

$$
\Lambda_{A}^{+}:=\Lambda_{A, 0}^{+}
$$

3.14 Lemma. Assume that $A \in L_{\mathrm{loc}}^{\infty}\left(\mathbb{R}^{3}, \mathbb{R}^{3}\right)$ and that $V$ fulfills Hypothesis 2 , Then there is some $C \in(0, \infty)$ such that, for all $\zeta \in C^{\infty}\left(\mathbb{R}^{3},[0,1]\right)$ which are constant outside some ball such that $\zeta V_{\mathrm{C}}$ is bounded,

$$
\left\|\left|D_{A}\right|^{1 / 2} \zeta\left(\Lambda_{A}^{+}-\Lambda_{A, V}^{+}\right)\right\| \leqslant C\left(\|\zeta V\|+\|\nabla \zeta\|_{\infty}\right)
$$

In particular, $\zeta \Lambda_{A, V}^{+} \varphi \in \mathcal{D}\left(\left|D_{A}\right|^{1 / 2}\right)$, for every $\varphi \in \mathcal{D}\left(D_{A}\right)$.

Proof: Due to (55) the norm in the statement (if it exists) is bounded from above by

$$
\sup _{\substack{\phi \in \mathcal{D}\left(\left|D_{A}\right|^{1 / 2}\right), \psi \in \mathscr{H} \\\|\phi\|=\|\psi\|=1}} \int_{\Gamma}\left|\left\langle\left|D_{A}\right|^{1 / 2} \phi \mid \zeta\left(R_{A}(z)-R_{A, V}(z)\right) \psi\right\rangle\right| \frac{|d z|}{\pi} .
$$


We next use (33), (43), and (45) to conclude that the asserted bound holds true.

We note the following trivial consequence of the previous lemma: Namely, we pick some $\theta \in C_{0}^{\infty}\left(\mathbb{R}^{3},[0,1]\right)$ with $\theta \equiv 1$ on $\mathcal{B}_{1}(0)$ and $\theta \equiv 0$ outside $\mathcal{B}_{2}(0)$, and set $\theta_{R}(x):=\theta(x / R)$, for $R \geqslant 1, x \in \mathbb{R}^{3}$. By virtue of Hypothesis 2 and Lemma 3.14 we then have, for every $\zeta$ as in the statement of Lemma 3.14,

$$
\left\|\left|D_{A}\right|^{1 / 2}\left(1-\theta_{R}\right) \zeta\left(\Lambda_{A}^{+}-\Lambda_{A, V}^{+}\right)\right\| \leqslant C\left(\left\|\left(1-\theta_{R}\right) V\right\|+\frac{\|\nabla \theta\|_{\infty}}{R}\right) \longrightarrow 0,
$$

as $R$ tends to infinity.

3.15 Corollary. Assume that $A \in L_{\text {loc }}^{\infty}\left(\mathbb{R}^{3}, \mathbb{R}^{3}\right)$ and that $V$ fulfills Hypothesis 2. Then there is some $C \in(0, \infty)$ such that, for every $\zeta \in C^{\infty}\left(\mathbb{R}^{3}, \mathbb{R}\right)$, which is constant outide some ball and such that $\zeta V_{\mathrm{C}}^{\mathrm{s}}=0$, and every $\varphi \in \mathscr{D}$,

$$
\begin{aligned}
& \left|\left\langle\varphi \mid \Lambda_{A, V}^{+} \zeta D_{A, V_{\mathrm{C}}} \zeta \Lambda_{A, V}^{+} \varphi\right\rangle-\left\langle\varphi \mid \Lambda_{A}^{+} \zeta D_{A} \zeta \Lambda_{A}^{+} \varphi\right\rangle\right| \\
& \quad \leqslant\left(\|\zeta V\|+\|\nabla \zeta\|_{\infty}\right) \inf _{0<\varepsilon \leqslant 1}\left\{\varepsilon\left\langle\varphi\left|\Lambda_{A}^{+} \zeta\right| D_{A} \mid \zeta \Lambda_{A}^{+} \varphi\right\rangle+\frac{C}{\varepsilon}\|\varphi\|^{2}\right\},
\end{aligned}
$$

and

$$
\begin{aligned}
& \left|\left\langle\varphi \mid \zeta \Lambda_{A, V}^{+} D_{A, V_{\mathrm{C}}} \Lambda_{A, V}^{+} \zeta \varphi\right\rangle-\left\langle\varphi \mid \zeta \Lambda_{A}^{+} D_{A} \Lambda_{A}^{+} \zeta \varphi\right\rangle\right| \\
& \quad \leqslant\left(\|\zeta V\|+\|\nabla \zeta\|_{\infty}\right) \inf _{0<\varepsilon \leqslant 1}\left\{\varepsilon\left\langle\varphi \mid \zeta \Lambda_{A}^{+} D_{A} \Lambda_{A}^{+} \zeta \varphi\right\rangle+\frac{C}{\varepsilon}\|\varphi\|^{2}\right\} .
\end{aligned}
$$

The last estimate still holds true (with a new constant $C$ ), if $D_{A}$ and $D_{A, V_{\mathrm{C}}}$ are replaced by $D_{A}-1$ and $D_{A, V_{\mathrm{C}}}-1$, respectively.

Proof: Let $\varphi \in \mathscr{D}$ and let $\theta_{R}$ be the cut-off function constructed in the paragraph preceeding (67). On account of Lemma 3.9 we know that $\Lambda_{A, V}^{+} \varphi \in H^{1 / 2}$ and, hence, we infer from Lemma 3.2 (c) that $\theta_{R} \zeta \Lambda_{A, V}^{+} \varphi \in H_{c}^{1 / 2}$ belongs to the domain of $D_{A}$. Applying also the formula appearing in (ii) of Lemma 3.1 and using $\zeta V_{\mathrm{C}}^{\mathrm{s}}=0$ we obtain

$$
\begin{aligned}
\left\langle\varphi \mid \Lambda_{A, V}^{+} \zeta D_{A, V_{\mathrm{C}}^{\mathrm{s}}} \zeta \Lambda_{A, V}^{+} \varphi\right\rangle & =\lim _{R \rightarrow \infty}\left\langle\theta_{R} \zeta \Lambda_{A, V}^{+} \varphi \mid D_{A, V_{\mathrm{C}}^{\mathrm{s}}} \zeta \Lambda_{A, V}^{+} \varphi\right\rangle \\
& =\lim _{R \rightarrow \infty}\left\langle D_{A} \theta_{R} \zeta \Lambda_{A, V}^{+} \varphi \mid \zeta \Lambda_{A, V}^{+} \varphi\right\rangle .
\end{aligned}
$$

Writing $\delta \Lambda^{+}:=\Lambda_{A, V}^{+}-\Lambda_{A}^{+}$we further get

$$
\begin{aligned}
& \left\langle D_{A} \theta_{R} \zeta \Lambda_{A, V}^{+} \varphi \mid \zeta \Lambda_{A, V}^{+} \varphi\right\rangle \\
& =\left\langle D_{A} \theta_{R} \zeta \Lambda_{A}^{+} \varphi \mid \zeta \Lambda_{A}^{+} \varphi\right\rangle+\left\langle D_{A} \theta_{R} \zeta \Lambda_{A}^{+} \varphi \mid \zeta \delta \Lambda^{+} \varphi\right\rangle \\
& \quad+\left\langle\theta_{R} \zeta \delta \Lambda^{+} \varphi \mid D_{A} \zeta \Lambda_{A}^{+} \varphi\right\rangle+\left\langle D_{A} \theta_{R} \zeta \delta \Lambda^{+} \varphi \mid \zeta \delta \Lambda^{+} \varphi\right\rangle .
\end{aligned}
$$


By virtue of Lemma 3.14 we know that $\zeta \delta \Lambda^{+} \varphi \in \mathcal{D}\left(\left|D_{A}\right|^{1 / 2}\right)$ and it is easy to see that $D_{A} \theta_{R} \zeta \Lambda_{A}^{+} \varphi \rightarrow D_{A} \zeta \Lambda_{A}^{+} \varphi$, as $R \rightarrow \infty$. Using also (67) we arrive at

$$
\begin{aligned}
& \left|\left\langle\varphi \mid \Lambda_{A, V}^{+} \zeta D_{A, V_{\mathrm{C}}^{\mathrm{s}}} \zeta \Lambda_{A, V}^{+} \varphi\right\rangle-\left\langle D_{A} \zeta \Lambda_{A}^{+} \varphi \mid \zeta \Lambda_{A}^{+} \varphi\right\rangle\right| \\
& \quad \leqslant 2\left\|\left|D_{A}\right|^{1 / 2} \zeta \Lambda_{A}^{+} \varphi\right\|\left\|\left|D_{A}\right|^{1 / 2} \zeta \delta \Lambda^{+} \varphi\right\|+\left\|\left|D_{A}\right|^{1 / 2} \zeta \delta \Lambda^{+} \varphi\right\|^{2} .
\end{aligned}
$$

Therefore, we obtain (68) by applying Lemma 3.14 once again. (69) follows from a straightforward combination of (68) and Corollary 3.8. The last statement of Corollary 3.15 follows from (69) and Lemma 3.14 .

\section{Exponential localization}

In this section we prove Theorem 2.4. To this end we adapt an argument from [1] and some useful improvements of the latter from [16] to our non-local situation. In the proof below we present the general strategy of the argument. In doing so we refer to three technical lemmata whose proofs are postponed to the end of this section. Throughout this section we always assume that the assumptions of Theorem 2.4 are fulfilled.

Proof of Theorem 2.4: Since $H_{N}$ is bounded from below we may suppose that inf $I>-\infty$. By assumption we have $\sup I<\mathscr{E}_{N-1}+1$. Moreover, we consider $H_{N}$ as an operator on the unprojected $N$-particle space $\mathscr{H}_{N}$. In this case we have to keep in mind that 0 becomes an infinitely degenerated eigenvalue of $H_{N}$. Our goal is to show that there are $b, C \in(0, \infty)$ such that $\int_{\mathbb{R}^{3 N}} e^{2 b|X|}|\Phi(X)|^{2} d X \leqslant C$, for all normalized $\Phi \in \operatorname{Ran}\left(E_{I}\left(H_{N}\right)\right)$ such that $\Phi=\mathcal{A}_{N} \Phi$ and $\Phi=\Lambda_{A, V}^{+, N} \Phi$. Borrowing an idea from [29] we simplify the problem by using the bounds

$$
e^{2 b|X|} \leqslant \max _{j=1, \ldots, N} e^{2 b \sqrt{N}\left|x_{j}\right|} \leqslant \sum_{j=1}^{N} e^{2 b \sqrt{N}\left|x_{j}\right|}, \quad X=\left(x_{1}, \ldots, x_{N}\right) \in\left(\mathbb{R}^{3}\right)^{N},
$$

and the anti-symmetry of $\Phi=\mathcal{A}_{N} \Phi$. (We are not aiming to derive good estimates on the decay rate here.) Indeed, it suffices to show that there exist $a, C^{\prime} \in(0, \infty)$ such that

$$
\int_{\mathbb{R}^{3 N}} e^{2 a\left|x_{N}\right|}|\Phi(X)|^{2} d X \leqslant C
$$

for all $\Phi=\mathcal{A}_{N} \Phi=\Lambda_{A, V}^{+, N} \Phi \in E_{I}\left(H_{N}\right),\|\Phi\|=1$. Then Theorem 2.4 holds true with $b=a / \sqrt{N}$. Furthermore, it suffices to show that (70) holds true with 
$a\left|x_{N}\right|$ replaced by $F\left(x_{N}\right)$, for every (bounded) $F: \mathbb{R}^{3} \rightarrow \mathbb{R}$ satisfying (52). This is in fact an obvious consequence of the monotone convergence theorem applied to the integrals $\int_{\mathbb{R}^{3 N}} e^{2 F_{n}\left(x_{N}\right)}|\Phi(X)|^{2} d X$ with $\Phi$ as above, where $F_{1}, F_{2}, \ldots$ is a suitable increasing sequence of functions satisfying (52) and converging to $a\left|x_{N}\right|$. Therefore, it suffices to find some $a>0$ such that

$$
\left\|\left(\mathcal{A}_{N-1} \otimes e^{F}\right) E_{I}\left(H_{N}\right)\left(\mathcal{A}_{N-1} \otimes \mathbb{1}\right) \Lambda_{A, V}^{+, N}\right\|<\infty,
$$

for every $F$ satisfying (52), where $\mathcal{A}_{N-1}$ denotes anti-symmetrization of the first $N-1$ variables and $e^{F}$ acts only on the $N^{\text {th }}$ electron variable.

We start by introducing a comparison operator. To this end we pick some $\chi \in C^{\infty}\left(\mathbb{R}^{3},[0,1]\right)$ such that $\chi \equiv 1$ outside $\mathcal{B}_{2}(0)$ and $\chi \equiv 0$ on $\mathcal{B}_{1}(0)$ and set $\chi_{R}:=\chi(\cdot / R)$ and $\bar{\chi}_{R}:=1-\chi_{R}$, for $R \geqslant 1$. Furthermore, we define orthogonal projections

$$
\begin{array}{rlrl}
P_{N-1} & :=\mathcal{A}_{N-1} \Lambda_{A, V}^{+, N-1}, & P_{N-1}^{\perp}=\mathbb{1}_{\mathscr{H}_{N-1}}-P_{N-1}, \\
Q_{N}:=\left(\mathcal{A}_{N-1} \otimes \mathbb{1}\right) \Lambda_{A, V}^{+, N}, & Q_{N}^{\perp}=\mathbb{1}_{\mathscr{H}_{N}}-Q_{N} .
\end{array}
$$

Then the comparison operator is defined, à-priori on the domain $\mathscr{D}_{N} \subset \mathscr{H}_{N}$, by

$$
\begin{aligned}
\widetilde{H}_{N}:= & Q_{N} H_{N} Q_{N}+H_{N-1}^{\mathcal{A}} \otimes \Lambda_{A, V}^{-}+\mathscr{E}_{N-1}^{\mathcal{A}} P_{N-1}^{\perp} \otimes \mathbb{1} \\
& +P_{N-1} \otimes \Lambda_{A, V}^{+}\left(1-\mathscr{E}_{1} \mathcal{A}\right) \bar{\chi}_{R} \Lambda_{A, V}^{+}+Q_{N}^{\perp} \\
= & H_{N-1}^{\mathcal{A}} \otimes \mathbb{1}+\mathscr{E}_{N-1}^{\mathcal{A}} P_{N-1}^{\perp} \otimes \mathbb{1} \\
& +P_{N-1} \otimes \Lambda_{A, V}^{+}\left\{D_{A, V_{\mathrm{C}}}+\left(1-\mathscr{E}_{1} \mathcal{A}\right) \bar{\chi}_{R}\right\} \Lambda_{A, V}^{+}+Q_{N}^{\perp} \\
& +\sum_{i=1}^{N-1} Q_{N} W_{i N} Q_{N} .
\end{aligned}
$$

We denote the Friedrichs extension of $\widetilde{H}_{N}$ again by the same symbol. Notice that on $\mathscr{D}_{N}$ we have $H_{N-1}^{\mathcal{A}} \otimes \mathbb{1}+\mathscr{E}_{N-1}^{\mathcal{A}} P_{N-1}^{\perp} \otimes \mathbb{1} \geqslant \mathscr{E}_{N-1}^{\mathcal{A}} \mathbb{1}_{\mathscr{H}_{N}}$. Furthermore, Lemma 4.3 below this implies that

$$
P_{N-1} \otimes \Lambda_{A, V}^{+}\left\{D_{A, V_{\mathrm{C}}}+\left(1-\mathscr{E}_{1}^{\mathcal{A}}\right) \bar{\chi}_{R}\right\} \Lambda_{A, V}^{+}+Q_{N}^{\perp} \geqslant \mathbb{1}-o(1) P_{N-1} \otimes \mathbb{1},
$$

as $R$ tends to infinity. We now pick some $\varepsilon>0$ with $\sup I<\mathscr{E}_{N-1}^{\mathcal{A}}+1-\varepsilon$. Then the above remarks imply

$$
\left\langle\psi \mid \widetilde{H}_{N} \psi\right\rangle \geqslant\left(\mathscr{E}_{N-1}^{\mathcal{A}}+1-\varepsilon / 2\right)\|\psi\|^{2}, \quad \psi \in \mathscr{D}_{N},
$$

for all sufficiently large $R \geqslant 1$. Next, we define

$$
H_{N}^{\prime}:=Q_{N} H_{N} Q_{N}+H_{N-1}^{\mathcal{A}} \otimes \Lambda_{A, V}^{-}
$$


Then $\widetilde{H}_{N}$ and $H_{N}^{\prime}$ have the same domain since they differ by a bounded operator on their common form core $\mathscr{D}_{N}$. We further pick some $\widetilde{\chi}_{I} \in C_{0}^{\infty}(\mathbb{R},[0,1])$, such that $\widetilde{\chi}_{I} \equiv 1$ on $I$ and $\operatorname{supp}\left(\widetilde{\chi}_{I}\right) \subset\left(-\infty, \mathscr{E}_{N-1}^{\mathcal{A}}+1-\varepsilon\right)$. Then $\widetilde{\chi}_{I}\left(\widetilde{H}_{N}\right)=0$ by (75) and we observe that

$$
Q_{N} E_{I}\left(H_{N}\right) Q_{N}=Q_{N} E_{I}\left(H_{N}^{\prime}\right) Q_{N}=\left(\widetilde{\chi}_{I}\left(H_{N}^{\prime}\right)-\widetilde{\chi}_{I}\left(\widetilde{H}_{N}\right)\right) Q_{N}
$$

We preserve the symbol $\tilde{\chi}_{I}$ to denote an almost analytic extension of $\tilde{\chi}_{I}$ (see, e.g., [12]) to a smooth, compactly supported function on the complex plane satisfying

$$
\begin{aligned}
& \operatorname{supp}\left(\widetilde{\chi}_{I}\right) \subset\left(-\infty, \mathscr{E}_{N-1}^{\mathcal{A}}+1-\varepsilon\right)+i(-\delta, \delta) \\
& \partial_{\bar{z}} \widetilde{\chi}_{I}(z)=\mathcal{O}_{N}\left(|\Im z|^{N}\right), \quad N \in \mathbb{N}
\end{aligned}
$$

where $\partial_{\bar{z}}=\frac{1}{2}\left(\partial_{\Re z}+i \partial_{\Im z}\right)$. Here we may choose $\delta>0$ as small as we please. We shall apply the Helffer-Sjöstrand formula (see, e.g., [12]),

$$
\tilde{\chi}_{I}(T)=\int_{\mathbb{C}}(z-T)^{-1} d \widetilde{\chi}_{I}(z), \quad d \widetilde{\chi}_{I}(z):=\frac{i}{2 \pi} \partial_{\bar{z}} \tilde{\chi}_{I}(z) d z \wedge d \bar{z},
$$

which holds for every self-adjoint operator $T$ on some Hilbert space. By means of (76) we then find the representation

$$
Q_{N} E_{I}\left(H_{N}\right) Q_{N}=\int_{\mathbb{C}}\left[\left(H_{N}^{\prime}-z\right)^{-1}-\left(\widetilde{H}_{N}-z\right)^{-1}\right] d \widetilde{\chi}_{I}(z) Q_{N} .
$$

For some $F$ as in (52) (which acts only on the last variable in what follows) we abbreviate

$$
\Lambda_{A, V}^{F, N}:=e^{F} \Lambda_{A, V}^{+, N} e^{-F} .
$$

Then (78) and the second resolvent identity together with the trivial identities $Q_{N}^{\perp} Q_{N}=0=\left(P_{N-1}^{\perp} \otimes \mathbb{1}\right) Q_{N}$ yield

$$
\begin{aligned}
& \left\|\left(\mathcal{A}_{N-1} \otimes e^{F}\right) E_{I}\left(H_{N}\right)\left(\mathcal{A}_{N-1} \otimes \mathbb{1}\right) \Lambda_{A, V}^{+, N}\right\| \\
& \leqslant \int_{\mathbb{C}} \| e^{F}\left(\widetilde{H}_{N}-z\right)^{-1} P_{N-1} \otimes\left\{\Lambda_{A, V}^{+}\left(1-\mathscr{E}_{1}^{\mathcal{A}}\right) \bar{\chi}_{R} \Lambda_{A, V}^{+}\right\} \times \\
& \times Q_{N}\left(H_{N}^{\prime}-z\right)^{-1} Q_{N} \|\left|d \widetilde{\chi}_{I}(z)\right| \\
& \leqslant\left(1-\mathscr{E}_{1}^{\mathcal{A}}\right) \int_{\mathbb{C}}\left\|e^{F}\left(\widetilde{H}_{N}-z\right)^{-1} e^{-F}\right\|\left\|\Lambda_{A, V}^{F, N}\right\|\left\|e^{F} \bar{\chi}_{R}\right\| \frac{\left|d \widetilde{\chi}_{I}(z)\right|}{|\Im z|} \\
& \leqslant C_{a, R} \int_{\mathbb{C}}\left\|e^{F}\left(\widetilde{H}_{N}-z\right)^{-1} e^{-F}\right\| \frac{\left|d \widetilde{\chi}_{I}(z)\right|}{|\Im z|} .
\end{aligned}
$$


In the last step we apply Proposition 3.6 and $\left\|e^{F} \bar{\chi}_{R}\right\| \leqslant e^{2 a R}$. By (77) $\left|d \widetilde{\chi}_{I}(z)\right| /|\Im z|$ is a finite measure. To conclude the proof of Theorem 2.4 it thus remains to show that the norm of $e^{F}\left(\widetilde{H}_{N}-z\right)^{-1} e^{-F}$ is uniformly bounded in all $z \in \operatorname{supp}\left(\tilde{\chi}_{I}\right) \backslash \mathbb{R}$ and $F$ satisfying (52). This is done in the rest of this proof.

Since $F$ satisfies (52) we know that $\mathbb{1}_{N-1} \otimes e^{F}$ is an isomorphism on $\mathscr{H}_{N}$. Therefore, the densely defined operators $e^{F} \widetilde{H}_{N} e^{-F}$ and $\widetilde{H}_{N}$ have the same resolvent set and

$$
\mathscr{R}_{F}(z):=e^{F}\left(\widetilde{H}_{N}-z\right)^{-1} e^{-F}=\left(e^{F} \widetilde{H}_{N} e^{-F}-z\right)^{-1}, \quad z \in \varrho\left(\widetilde{H}_{N}\right) .
$$

In particular, $e^{F} \widetilde{H}_{N} e^{-F}$ is closed because its resovent set is not empty. Using the identity $\mathscr{R}_{F}(z)^{*^{-1}}=\mathscr{R}_{F}(z)^{-1^{*}}$ we readily verify that $\left(e^{F} \widetilde{H}_{N} e^{-F}\right)^{*}=$ $e^{-F} \widetilde{H}_{N} e^{F}$. Since $e^{ \pm F}$ maps $\mathscr{D}_{N}$ into itself we further have

$$
\mathscr{D}_{N} \subset \mathcal{D}\left(e^{ \pm F} \widetilde{H}_{N} e^{\mp F}\right)=e^{ \pm F} \mathcal{D}\left(\widetilde{H}_{N}\right) \subset e^{ \pm F} \mathcal{Q}\left(\widetilde{H}_{N}\right)
$$

The following two lemmata, whose proofs are postponed to the end of this section, show that $e^{F} \widetilde{H}_{N} e^{-F}$ is a small form perturbation of $\widetilde{H}_{N}$. We define $T: \mathscr{D}_{N} \rightarrow \mathscr{H}_{N}$ by

$$
T \varphi:=e^{F} \widetilde{H}_{N} e^{-F} \varphi-\widetilde{H}_{N} \varphi, \quad \varphi \in \mathscr{D}_{N} .
$$

4.1 Lemma. Assume that $F: \mathbb{R}^{3} \rightarrow \mathbb{R}$ satisfies (52). Then we have, as $a>0$ tends to zero,

$$
|\langle\varphi \mid T \varphi\rangle| \leqslant a\left\langle\varphi \mid \widetilde{H}_{N} \varphi\right\rangle+\mathcal{O}(a)\langle\varphi \mid \varphi\rangle, \quad \varphi \in \mathscr{D}_{N} .
$$

4.2 Lemma. There exist constants $c_{1}, c_{2} \in(0, \infty)$ such that, for all $F: \mathbb{R}^{3} \rightarrow \mathbb{R}$ satisfying (52) and all $\varphi \in \mathscr{D}_{N}$,

$$
\left|\left\langle e^{ \pm F} \varphi \mid \widetilde{H}_{N} e^{ \pm F} \varphi\right\rangle\right| \leqslant c_{1}\left\|e^{ \pm F}\right\|^{2}\left\langle\varphi \mid \widetilde{H}_{N} \varphi\right\rangle+c_{2}\left\|e^{ \pm F}\right\|^{2}\|\varphi\|^{2} .
$$

In particular, $e^{ \pm F} \mathcal{Q}\left(\widetilde{H}_{N}\right) \subset \mathcal{Q}\left(\widetilde{H}_{N}\right)$.

If $a<1 / 2$ then Lemma 4.1 implies that $\left(e^{F} \widetilde{H}_{N} e^{-F}\right) \Upsilon_{\mathscr{D}_{N}}$ has a distinguished, sectorial, closed extension, $\widetilde{H}_{N}^{F}$, that is the only closed extension having the properties $\mathcal{D}\left(\widetilde{H}_{N}^{F}\right) \subset \mathcal{Q}\left(\widetilde{H}_{N}\right), \mathcal{D}\left(\widetilde{H}_{N}^{F *}\right) \subset \mathcal{Q}\left(\widetilde{H}_{N}\right)$, and $i \eta \in \varrho\left(\widetilde{H}_{N}^{F}\right)$, for all $\eta \in \mathbb{R}$ with sufficiently large absolute value; see [24]. Thanks to (80), (81), and Lemma 4.2, we know that $e^{F} \widetilde{H}_{N} e^{-F}$ is a closed extension enjoying these properties, whence

$$
\widetilde{H}_{N}^{F}=e^{F} \widetilde{H}_{N} e^{-F}
$$


We are now prepared to derive a uniform bound on the norm under the integral sign in (79). For $z \in \operatorname{supp}\left(\tilde{\chi}_{I}\right)$ and $\varphi \in \mathscr{D}_{N}$, we obtain

$$
\begin{aligned}
\Re\left\langle\varphi \mid\left(\widetilde{H}_{N}^{F}-z\right) \varphi\right\rangle & =\left\langle\varphi \mid\left(\widetilde{H}_{N}-\Re z\right) \varphi\right\rangle+\Re\langle\varphi \mid T \varphi\rangle \\
& \geqslant(1-a)\left\langle\varphi \mid\left(\widetilde{H}_{N}-\frac{\Re z}{1-a}\right) \varphi\right\rangle-\mathcal{O}(a)\|\varphi\|^{2} .
\end{aligned}
$$

By (75) and (77) we thus find $a \in(0,1 / 2)$ and $R \in[1, \infty)$ such that, for all $z \in \operatorname{supp}\left(\widetilde{\chi}_{I}\right)$ and $\varphi \in \mathscr{D}_{N}$,

$$
\Re\left\langle\varphi \mid\left(\widetilde{H}_{N}^{F}-z\right) \varphi\right\rangle \geqslant \frac{\varepsilon}{4}\|\varphi\|^{2} .
$$

This inequality implies that, for $z \in \operatorname{supp}\left(\widetilde{\chi}_{I}\right)$, the numerical range of $\widetilde{H}_{N}^{F}-z$ is contained in the half space $\{\zeta \in \mathbb{C}: \Re \zeta \geqslant \varepsilon / 4\}$ [24, Theorem VI.1.18 and Corollary VI.2.3]. Moreover, by (80) the deficiency of $\widetilde{H}_{N}^{F}-z$ is zero, for all $z \in \mathbb{C} \backslash \mathbb{R}$, and we may hence estimate the norm of $\left(\widetilde{H}_{N}^{F}-z\right)^{-1}$ by the inverse distance of $z$ to the numerical range of $\widetilde{H}_{N}^{F}$ [24, Theorem V.3.2]. We thus arrive at

$$
\left\|\left(\widetilde{H}_{N}^{F}-z\right)^{-1}\right\| \leqslant \frac{4}{\varepsilon}, \quad z \in \operatorname{supp}\left(\widetilde{\chi}_{I}\right)
$$

which together with (79) proves Theorem 2.4.

4.3 Lemma. For every sufficiently large $R \geqslant 1$, there is some $c_{R} \in(0, \infty)$ such that $c_{R} \rightarrow 0$, as $R \rightarrow \infty$, and, for all $\varphi \in \mathscr{D}$,

$$
\left\langle\varphi \mid \Lambda_{A, V}^{+}\left[D_{A, V_{\mathrm{C}}}+\left(1-\mathscr{E}_{1}^{\mathcal{A}}\right) \bar{\chi}_{R}\right] \Lambda_{A, V}^{+} \varphi\right\rangle \geqslant\left\|\Lambda_{A, V}^{+} \varphi\right\|^{2}-c_{R}\|\varphi\|^{2} .
$$

Proof: To begin with we introduce a scaled partition of unity. Namely, we pick some $\tilde{\mu} \in C_{0}^{\infty}\left(\mathbb{R}^{3},[0,1]\right)$ such that $\tilde{\mu} \equiv 1$ on $\mathcal{B}_{2}(0)$ and observe that $\theta:=$ $\tilde{\mu}^{2}+(1-\tilde{\mu})^{2}$ is strictly positive. We further set, for $R \geqslant 1$ and $x \in \mathbb{R}^{3}, \mu_{1}(x) \equiv$ $\mu_{R, 1}(x):=\tilde{\mu}(x / R) / \theta^{1 / 2}(x / R)$, and $\mu_{2}(x) \equiv \mu_{R, 2}(x):=(1-\tilde{\mu}(x / R)) / \theta^{1 / 2}(x / R)$, so that $\mu_{1}^{2}+\mu_{2}^{2}=1$. Since $\mu_{1} \nabla \mu_{1}+\mu_{2} \nabla \mu_{2}=\nabla\left(\mu_{1}^{2}+\mu_{2}^{2}\right) / 2=0$ it follows that, for $\varphi \in \mathscr{D}$,

$$
\begin{aligned}
& \left\langle\varphi \mid \Lambda_{A, V}^{+}\left[D_{A, V_{\mathrm{C}}}+\left(1-\mathscr{E}_{1}^{\mathcal{A}}\right) \bar{\chi}_{R}\right] \Lambda_{A, V}^{+} \varphi\right\rangle \\
& =\sum_{j=1,2}\left\langle\varphi \mid \Lambda_{A, V}^{+}\left[\mu_{j} D_{A, V_{\mathrm{C}}} \mu_{j}+\left(1-\mathscr{E}_{1}^{\mathcal{A}}\right) \mu_{j}^{2} \bar{\chi}_{R}\right] \Lambda_{A, V}^{+} \varphi\right\rangle=: \sum_{j=1,2} Y_{j} .
\end{aligned}
$$

To treat the summand with $j=1$ we use that, by construction, $\mu_{1} \bar{\chi}_{R}=\mu_{1}$, for every $R \geqslant 1$. Taking also Corollary 3.8 and (21) into account we find, for all 
$R \geqslant 1$ and $\varphi \in \mathscr{D}$

$$
\begin{aligned}
Y_{1} \geqslant & (1-1 / R)\left\langle\mu_{1} \varphi \mid \Lambda_{A, V}^{+}\left[D_{A, V_{\mathrm{C}}}-\mathscr{E}_{1}^{\mathcal{A}}\right] \Lambda_{A, V}^{+} \mu_{1} \varphi\right\rangle \\
& +\left\|\mu_{1} \Lambda_{A, V}^{+} \varphi\right\|^{2}-\mathcal{O}(1 / R)\|\varphi\|^{2} \\
\geqslant & \left\|\mu_{1} \Lambda_{A, V}^{+} \varphi\right\|^{2}-\mathcal{O}(1 / R)\|\varphi\|^{2} .
\end{aligned}
$$

We next turn to the summand with $j=2$ in (87) where $\mu_{2}^{2} \bar{\chi}_{R}=0$. Applying successively Corollaries 3.8 and 3.15, Proposition [3.6, and Lemma 3.14 we deduce that, for all $\varphi \in \mathscr{D}$ and every $\varepsilon>0$,

$$
\begin{aligned}
\left\langle\varphi \mid \Lambda_{A, V}^{+} \mu_{2} D_{A, V_{\mathrm{C}}} \mu_{2} \Lambda_{A, V}^{+} \varphi\right\rangle & \geqslant(1-\varepsilon)\left\langle\varphi \mid \mu_{2} \Lambda_{A}^{+} D_{A} \Lambda_{A}^{+} \mu_{2} \varphi\right\rangle-o_{\varepsilon}(1)\|\varphi\|^{2} \\
& \geqslant(1-\varepsilon)\left\|\Lambda_{A}^{+} \mu_{2} \varphi\right\|^{2}-o_{\varepsilon}(1)\|\varphi\|^{2} \\
& \geqslant(1-\varepsilon)^{2}\left\|\mu_{2} \Lambda_{A}^{+} \varphi\right\|^{2}-o_{\varepsilon}(1)\|\varphi\|^{2} \\
& \geqslant(1-\varepsilon)^{3}\left\|\mu_{2} \Lambda_{A, V}^{+} \varphi\right\|^{2}-o_{\varepsilon}(1)\|\varphi\|^{2}
\end{aligned}
$$

as $R \rightarrow \infty$. We conclude by combining (87)-(89) and using $\mu_{1}^{2}+\mu_{2}^{2}=1$.

Proof of Lemma 4.1: We have to study the contribution to $T=e^{F} \widetilde{H}_{N} e^{-F}-\widetilde{H}_{N}$ coming from each term in (72)-(74). The terms in (72) commute with $e^{F}$ and hence give no contribution. In order to estimate the contribution coming from the left term in (73) we first observe that Corollary 3.7 implies the following identity on $\mathscr{D}$,

$$
\begin{aligned}
e^{F} \Lambda_{A, V}^{+} D_{A, V_{\mathrm{C}}} \Lambda_{A, V}^{+} e^{-F} & =\Lambda_{A, V}^{F}\left(D_{A, V_{\mathrm{C}}}+i \alpha \cdot \nabla F\right) \Lambda_{A, V}^{F} \\
& =\Lambda_{A, V}^{F} D_{A, V_{\mathrm{C}}} \Lambda_{A, V}^{F}+\mathcal{O}(a) .
\end{aligned}
$$

The term in (73) involving the cut-off function $\bar{\chi}_{R}$ yields a contribution of order $\mathcal{O}(a)$, too, due to Corollary 3.8. To account for the projection on the right in (73) we write $Q_{N}^{\perp}=\mathbb{1}_{\mathscr{H}_{N}}-P_{N-1} \otimes \Lambda_{A, V}^{+}$and use Proposition 3.6 to obtain $\left\|Q_{N}^{\perp}-e^{F} Q_{N}^{\perp} e^{-F}\right\|=\mathcal{O}(a)$. Finally, we apply Corollary 3.8 to (90) and Corollary 3.12 to all terms in (74) - this is the only place in this section where we use the assumption that $B$ is bounded - and arrive at

$$
|\langle\varphi \mid T \varphi\rangle| \leqslant a\left\langle\varphi \mid Q_{N}\left\{D_{A, V_{\mathrm{C}}}^{(N)}+\sum_{i=1}^{N-1} W_{i N}\right\} Q_{N} \varphi\right\rangle+\mathcal{O}(a)\|\varphi\|^{2} .
$$

Since $H_{N-1}^{\mathcal{A}} \geqslant \mathscr{E}_{N-1}^{\mathcal{A}}$ this completes the proof of Lemma 4.1.

Proof of Lemma 4.2: We drop the \pm -signs in (84) since the they do not play any role in this proof. It is clear that we only have to comment on those terms 
in (72)-(74) that involve unbounded operators. Since $H_{N-1} \otimes \mathbb{1}$ commutes with $e^{F}$ and since $H_{N-1} \geqslant \mathscr{E}_{N-1}^{\mathcal{A}}$ we first find, for $\varphi \in \mathscr{D}_{N}$,

$$
\begin{aligned}
\left\langle e^{F} \varphi\right| & \left.\left(H_{N-1} \otimes \mathbb{1}-\mathscr{E}_{N-1}^{\mathcal{A}} P_{N-1}^{\perp} \otimes \mathbb{1}\right) e^{F} \varphi\right\rangle \\
& \leqslant\left\|e^{2 F}\right\|\left\langle\varphi \mid\left(H_{N-1} \otimes \mathbb{1}-\mathscr{E}_{N-1}^{\mathcal{A}} P_{N-1}^{\perp} \otimes \mathbb{1}\right) \varphi\right\rangle .
\end{aligned}
$$

By virtue of Proposition 3.11 we can estimate $\left|\left\langle\varphi \mid e^{F} Q_{N} W_{i N} Q_{N} e^{F} \varphi\right\rangle\right|$, for $\varphi \in \mathscr{D}_{N}$, as

$$
\begin{aligned}
\left\|W_{i N}^{1 / 2} Q_{N} e^{F} \varphi\right\|^{2} & \leqslant 2\left\|e^{F} W_{i N}^{1 / 2} Q_{N} \varphi\right\|^{2}+2\left\|W_{i N}^{1 / 2}\left[e^{F}, Q_{N}\right] e^{-F} e^{F} \varphi\right\|^{2} \\
& \leqslant 2\left\|e^{F}\right\|^{2}\left\|W_{i N}^{1 / 2} Q_{N} \varphi\right\|^{2}+\mathcal{O}\left(a^{2}\right)\left\|e^{F}\right\|^{2}\|\varphi\|^{2} .
\end{aligned}
$$

(If $B$ is unbounded, then the $\mathcal{O}$-symbol in (92) depends on the supremum of $|B|$ on $\operatorname{supp}(\nabla F)$.) It remains to prove that there are constants $c_{3}, c_{4} \in(0, \infty)$ such that

$$
\begin{aligned}
\langle\varphi| e^{F} \Lambda_{A, V}^{+} & \left.D_{A, V_{\mathrm{C}}} \Lambda_{A, V}^{+} e^{F} \varphi\right\rangle \\
& \leqslant c_{3}\left\|e^{F}\right\|^{2}\left\langle\varphi \mid \Lambda_{A, V}^{+} D_{A, V_{\mathrm{C}}} \Lambda_{A, V}^{+} \varphi\right\rangle+c_{4}\left\|e^{F}\right\|^{2}\|\varphi\|^{2},
\end{aligned}
$$

for $\varphi \in \mathscr{D}$. Moreover, since $V_{\mathrm{H}}$ and $V_{\mathrm{E}}$ are bounded it suffices to prove this estimate with $D_{A, V_{\mathrm{C}}}$ replaced by $\widetilde{D}:=D_{A, V}-e_{0}$, which is positive on the range of $\Lambda_{A, V}^{+}$. We abbreviate $\Lambda^{ \pm}:=\Lambda_{A, V}^{ \pm}$in the rest of this proof and we seek for bounds on both terms on the right side of

$$
\left\|\left(\widetilde{D} \Lambda^{+}\right)^{1 / 2} e^{F} \varphi\right\|^{2} \leqslant 2 \sum_{\sharp= \pm}\left\|\left(\widetilde{D} \Lambda^{+}\right)^{1 / 2} e^{F} \Lambda^{\sharp} \varphi\right\|^{2} .
$$

Here the norm with $\sharp=-$ equals $\left\|\left(\widetilde{D} \Lambda^{+}\right)^{1 / 2}\left[e^{F}, \Lambda^{-}\right] \varphi\right\|$ and is not greater than some $\mathcal{O}(a)\left\|e^{F}\right\|\|\varphi\|$ due to Proposition 3.6. We next define

$$
\hat{D}:=\Lambda^{+}\left(D_{A, V}-e_{0}\right) \Lambda^{+}+\mathbb{1}=\Lambda^{+} \widetilde{D} \Lambda^{+}+\mathbb{1} \geqslant \mathbb{1} .
$$

In fact, because of $\left\|\left(\widetilde{D} \Lambda^{+}\right)^{1 / 2} \hat{D}^{-1 / 2}\right\| \leqslant 1$ and

$$
\begin{aligned}
\left\|\hat{D}^{1 / 2} e^{F} \Lambda^{+} \varphi\right\|^{2} & \leqslant\left\|e^{F} \hat{D}^{1 / 2} \Lambda^{+} \varphi\right\|^{2}+\left\|\left[\hat{D}^{1 / 2}, e^{F}\right] \Lambda^{+} \varphi\right\|^{2} \\
& \leqslant\left\|e^{F}\right\|^{2}\left\|\Lambda^{+} \hat{D}^{1 / 2} \varphi\right\|^{2}+\left\|\hat{D}^{1 / 2}\left[\hat{D}^{-1 / 2}, e^{F}\right] \Lambda^{+} \hat{D}^{1 / 2} \varphi\right\|^{2}
\end{aligned}
$$

we shall see that (93) holds true as soon as we have shown that

$$
\left\|\hat{D}^{1 / 2}\left[\hat{D}^{-1 / 2}, e^{F}\right] \Lambda^{+}\right\|=\mathcal{O}(a)\left\|e^{F}\right\| .
$$


To check whether (95) is correct we first note that, on $\mathscr{D}$,

$$
\begin{aligned}
{\left[\hat{D}, e^{F}\right] } & =\Lambda^{+}\left[\widetilde{D}, e^{F}\right]+\left[\Lambda^{+}, e^{F}\right] \widetilde{D} \\
& =-\Lambda^{+} i \alpha \cdot \nabla F e^{F}+\left(\left[V_{\mathrm{E}}, e^{F}\right] e^{-F}\right) e^{F}+\left[\Lambda^{+}, e^{F}\right] \widetilde{D}
\end{aligned}
$$

We apply the norm-convergent integral representation

$$
T^{-1 / 2}=\frac{1}{\pi} \int_{0}^{\infty} \frac{1}{T+t} \frac{d t}{\sqrt{t}},
$$

which holds for any strictly positive operator, $T$, on some Hilbert space. For $\phi, \psi \in \mathscr{D}$, it implies

$$
\left\langle\hat{D}^{1 / 2} \phi \mid\left[\hat{D}^{-1 / 2}, e^{F}\right] \Lambda^{+} \psi\right\rangle=\frac{1}{\pi} \int_{0}^{\infty}\left\langle\hat{D}^{1 / 2} \phi \mid \frac{-1}{\hat{D}+t}\left[\hat{D}, e^{F}\right] \frac{\Lambda^{+}}{\hat{D}+t} \psi\right\rangle \frac{d t}{\sqrt{t}} .
$$

We estimate the contribution of the first term on the right side of (96) to (98) as

$$
\left|\left\langle\hat{D}^{1 / 2} \phi \mid \frac{\Lambda^{+}}{\hat{D}+t} i \alpha \cdot \nabla F e^{F} \frac{\Lambda^{+}}{\hat{D}+t} \psi\right\rangle\right| \leqslant \frac{\mathcal{O}(a)\left\|e^{F}\right\|}{(1+t)^{3 / 2}}\|\phi\|\|\psi\|, \quad t \geqslant 0 .
$$

In view of (12) the second term in (96) can be dealt with similarly. To account for the second term in (96) we apply Proposition 3.6 and obtain, for $t \geqslant 0$,

$$
\left|\left\langle\phi \mid \frac{1}{\hat{D}+t}\left\{\hat{D}^{1 / 2}\left[\Lambda^{+}, e^{F}\right] e^{-F}\right\} e^{F} \frac{\widetilde{D}}{\hat{D}+t} \Lambda^{+} \psi\right\rangle\right| \leqslant \frac{\mathcal{O}(a)\left\|e^{F}\right\|}{1+t}\|\phi\|\|\psi\| .
$$

Equations (98)-(100) show that (95) holds true, which completes the proof of Lemma 4.2.

\section{The lower bound on $\inf \sigma_{\text {ess }}\left(H_{N}\right)$}

In order to prove the 'hard part' of the HVZ theorem, Theorem 2.7(ii), we employ an idea we learned from [16]: One may use a localization estimate for spectral projections to prove their compactness. Adapted to our non-local model the argument looks as follows:

5.1 Theorem. Let the assumptions of Theorem [2.7(ii) be fulfilled and let $I \subset \mathbb{R}$ be an interval sup $I<1+\mathscr{E}_{N-1}^{\mathcal{A}}$. Then the spectral projection $E_{I}\left(H_{N}^{\mathcal{A}}\right)$ is a compact operator on $\mathcal{A}_{N} \mathscr{H}_{N}^{+}$. In particular,

$$
\sigma_{\mathrm{ess}}\left(H_{N}^{\mathcal{A}}\right) \subset\left[1+\mathscr{E}_{N-1}^{\mathcal{A}}, \infty\right) .
$$


Proof: Let $g \in C(\mathbb{R},(0, \infty))$ satisfy $g(r) \rightarrow \infty, r \rightarrow \infty$, and $g(|X|) E_{I}\left(H_{N}^{\mathcal{A}}\right) \in$ $\mathscr{L}\left(\mathcal{A}_{N} \mathscr{H}_{N}^{+}\right)$and set $h:=1 / g$. We let $\varrho_{R}$ denote a smoothed characteristic function of the closed ball in $\mathbb{R}^{3}$ with radius $R>0$ and center 0 and set $\chi_{R}(X):=\varrho_{R}\left(x_{1}\right) \cdots \varrho_{R}\left(x_{N}\right)$, for $X=\left(x_{1}, \ldots, x_{N}\right) \in\left(\mathbb{R}^{3}\right)^{N}$. First, we argue that it suffices to show that $E_{I}\left(H_{N}^{\mathcal{A}}\right) \chi_{R}(-\Delta+1)^{1 / 8}$ is a (densely defined) bounded operator from $\mathscr{H}_{N}$ to $\mathcal{A}_{N} \mathscr{H}_{N}^{+}$. In fact, let us assume that this is the case. Since $(-\Delta+1)^{-1 / 8} h(|X|)$ is compact and $g(|X|) E_{I}\left(H_{N}^{\mathcal{A}}\right)$ is bounded, it then follows that

$$
\begin{aligned}
& E_{I}\left(H_{N}^{\mathcal{A}}\right)\left[\chi_{R} h(|X|)\right] g(|X|) E_{I}\left(H_{N}^{\mathcal{A}}\right) \\
& \quad=E_{I}\left(H_{N}^{\mathcal{A}}\right) \chi_{R}(-\Delta+1)^{1 / 8}\left[(-\Delta+1)^{-1 / 8} h(|X|)\right] g(|X|) E_{I}\left(H_{N}^{\mathcal{A}}\right)
\end{aligned}
$$

is compact. Since $\chi_{R} h(|X|)$ converges to $h(|X|)$ in the operator norm, as $R$ tends to infinity, it further follows that $E_{I}\left(H_{N}^{\mathcal{A}}\right)=E_{I}\left(H_{N}^{\mathcal{A}}\right) h(|X|) g(|X|) E_{I}\left(H_{N}^{\mathcal{A}}\right)$ is compact, too.

To verify that $E_{I}\left(H_{N}^{\mathcal{A}}\right) \chi_{R}(-\Delta+1)^{1 / 8}$ is bounded we set $S:=1+\sum_{j=1}^{N}\left|D_{A, V}^{(j)}\right|$ and write, for some sufficiently large $c>0$,

$$
\begin{aligned}
& E_{I}\left(H_{N}^{\mathcal{A}}\right) \chi_{R}(-\Delta+1)^{1 / 8}=E_{I}\left(H_{N}^{\mathcal{A}}\right)\left(H_{N}+c\right)^{1 / 2} \times \\
& \quad \times\left\{\left(H_{N}+c\right)^{-1 / 2} \Lambda_{A, V}^{+, N} S^{1 / 2}\right\}\left\{S^{-1 / 2} \chi_{R}(-\Delta+1)^{1 / 8}\right\} .
\end{aligned}
$$

Here the left curly bracket in (101) is a bounded operator from $\mathscr{H}_{N}$ to $\mathscr{H}_{N}^{+}$ since $\Lambda_{A, V}^{+, N} S \Lambda_{A, V}^{+, N} \leqslant H_{N}+c$, provided $c$ is large enough, due to the positivity of the interaction potentials and the boundedness of $V_{\mathrm{H}}$ and $V_{\mathrm{E}}$. To see that the right curly bracket in (101) is a bounded operator in $\mathscr{H}_{N}$ we first notice that it is a restriction of $S^{-1 / 2} T^{*}$, where $T:=(-\Delta+1)^{1 / 8} \chi_{R}$ is closed. It thus remains to show that $T S^{-1 / 2}=T^{* *} S^{-1 / 2}=\left(S^{-1 / 2} T^{*}\right)^{*}$ belongs to $\mathscr{L}\left(\mathscr{H}_{N}\right)$. To this end we recall that $\left(-\Delta^{(i)}+1\right)^{1 / 4} \varrho_{R}^{(i)}\left(\left|D_{A, V}^{(i)}\right|+1\right)^{-1}$ is bounded on $L^{2}\left(\mathbb{R}_{i}^{3}, \mathbb{C}^{4}\right)$ since $\mathcal{D}\left(D_{A, V}\right) \subset H_{\text {loc }}^{1 / 2}\left(\mathbb{R}^{3}, \mathbb{C}^{4}\right)$. It follows that

$$
\left(-\Delta^{(i)}+1\right)^{1 / 4} \chi_{R} S^{-1}=\left(-\Delta^{(i)}+1\right)^{1 / 4} \chi_{R}\left(\left|D_{A, V}^{(i)}\right|+1\right)^{-1}\left(\left|D_{A, V}^{(i)}\right|+1\right) S^{-1}
$$

is bounded, for $i=1, \ldots, N$, and, hence, $\chi_{R}(-\Delta+1)^{1 / 4} \chi_{R} S^{-1} \in \mathscr{L}\left(\mathscr{H}_{N}\right)$. Since $\chi_{R}(-\Delta+1)^{1 / 4} \chi_{R}$ is a restriction of $T^{*} T$ we see that $T^{*} T S^{-1} \in \mathscr{L}\left(\mathscr{H}_{N}\right)$, which implies $|T| S^{-1 / 2} \in \mathscr{L}\left(\mathscr{H}_{N}\right)$ and, hence, $T S^{-1 / 2} \in \mathscr{L}\left(\mathscr{H}_{N}\right)$.

\section{$6 \quad$ Weyl sequences}

In this section we prove the 'easy part' of our HVZ theorem, namely Part (i) of Theorem 2.7 asserting that

$$
\sigma_{\text {ess }}\left(H_{N}^{\mathcal{A}}\right) \supset\left[\mathscr{E}_{N-1}^{\mathcal{A}}+1, \infty\right) .
$$


To this end we fix some spectral parameter $\lambda \geqslant 1$ throughout the whole section and $\left\{\psi_{n}\right\}_{n \in \mathbb{N}}$ will always denote a corresponding Weyl sequence as in Hypothesis $4(\mathrm{i})$.

In this and the following section we shall repeatedly employ the following sequence of cut-off functions: We pick some $\chi \in C^{\infty}(\mathbb{R},[0,1])$ such that $\chi \equiv 0$ on $(-\infty, 1-\varepsilon / 4]$ and $\chi \equiv 1$ on $[1, \infty)$. Here $\varepsilon \in(0,1)$ is a fixed parameter whose value becomes important only in Section 7. We set $\chi_{n}:=\chi\left(|x| / R_{n}\right)$, for $x \in \mathbb{R}^{3}$ and $n \in \mathbb{N}$, where $R_{n}$ is given by Hypothesis $4(\mathrm{i})$. Then it holds $\chi_{n} \psi_{n}=\psi_{n}$ and $\left\|\nabla \chi_{n}\right\|_{\infty}=R_{n}^{-1}\|\nabla \chi\|_{\infty} \rightarrow 0$, as $n \rightarrow \infty$.

To begin with we draw two simple conclusions from our hypotheses:

6.1 Lemma. Assume that $A \in L_{\mathrm{loc}}^{\infty}\left(\mathbb{R}^{3}, \mathbb{R}^{3}\right)$ and $V$ fulfill Hypotheses $4(i)$ and 2., respectively. Then

$$
\lim _{n \rightarrow \infty}\left\|\left(D_{A, V}-\lambda\right) \psi_{n}\right\|=0, \quad \lim _{n \rightarrow \infty}\left\|\left(D_{A, V_{\mathrm{C}}}-\lambda\right) \Lambda_{A, V}^{+} \psi_{n}\right\|=0
$$

Proof: The first identity is clear from the hypotheses. To treat the second we employ the cut-off functions defined in the paragraph preceeding the statement of this lemma and abbreviate $V_{\mathrm{HE}}:=V_{\mathrm{H}}+V_{\mathrm{E}}$. By means of Proposition 3.6 and $\left\|V_{\mathrm{HE}} \chi_{n}\right\| \rightarrow 0$ we then obtain

$$
\left\|V_{\mathrm{HE}} \Lambda_{A, V}^{+} \psi_{n}\right\| \leqslant\left\|V_{\mathrm{HE}} \chi_{n} \Lambda_{A, V}^{+} \psi_{n}\right\|+\left\|V_{\mathrm{HE}}\left[\Lambda_{A, V}^{+}, \chi_{n}\right] \psi_{n}\right\| \longrightarrow 0
$$

as $n$ tends to infinity. Therefore, the second identity follows from the first.

6.2 Lemma. Assume that $A \in L_{\mathrm{loc}}^{\infty}\left(\mathbb{R}^{3}, \mathbb{R}^{3}\right)$ and $V$ fulfill Hypotheses 4 (i) and 2 , respectively. Let $\varepsilon>0$ and set $I_{\varepsilon}:=(\lambda-\varepsilon, \lambda+\varepsilon)$. Then we have, as $n$ tends to infinity,

$$
\left\|E_{I_{\varepsilon}}\left(D_{A, V}\right) \psi_{n}\right\| \rightarrow 1, \quad \text { in particular, }\left\|\Lambda_{A, V}^{+} \psi_{n}\right\| \rightarrow 1 \text {. }
$$

Proof: Clearly, $\left\|E_{I_{\varepsilon}}\left(D_{A, V}\right) \psi_{n}\right\| \leqslant 1$ since $\psi_{n}$ is normalized. Suppose that there is some $\delta>0$ such that liminf $\left\|E_{I_{\varepsilon}}\left(D_{A, V}\right) \psi_{n}\right\|^{2} \leqslant 1-\delta$. Then we have $\lim _{\ell \rightarrow \infty}\left\|E_{I_{\varepsilon}}\left(D_{A, V}\right) \psi_{n_{\ell}}\right\|^{2} \leqslant 1-\delta$, for an appropriate subsequence, and

$$
\begin{gathered}
\lim _{\ell \rightarrow \infty}\left\|\left(D_{A, V}-\lambda\right) \psi_{n_{\ell}}\right\|^{2} \geqslant \varepsilon^{2} \liminf _{\ell \rightarrow \infty} \int_{\mathbb{R}}\left(1-\mathbb{1}_{I_{\varepsilon}}(s)\right) d\left\|E_{s}\left(D_{A}\right) \psi_{n_{\ell}}\right\|^{2} \\
=\varepsilon^{2}-\varepsilon^{2} \lim _{\ell \rightarrow \infty}\left\|E_{I}\left(D_{A, V}\right) \psi_{n_{\ell}}\right\|^{2} \geqslant \varepsilon^{2} \delta>0 .
\end{gathered}
$$

This is a contradiction to (102). 
In the following we show that $\mathscr{E}_{N-1}^{\mathcal{A}}+\lambda \in \sigma_{\text {ess }}\left(H_{N}\right)$ by means of a suitable Weyl sequence. Instead of applying Weyl's criterion directly to $H_{N}$ we shall, however, use a slightly strengthend version of it in Lemma 6.3 (see, e.g., [11]) which allows to work with quadratic forms. This is important since, for instance, it seems that one cannot expect Proposition 3.11 to hold with $W^{1 / 2}$ replaced by $W$. (At least not for large nuclear charges $Z \geqslant 118$.) To construct the Weyl sequence we pick, for every $n \in \mathbb{N}$, some

$$
\Phi_{n}=\mathcal{A}_{N-1} \Phi_{n} \in \Lambda_{A, V}^{+, N-1} \mathscr{D}_{N-1} \text { such that }\left\{\begin{array}{l}
\left\langle\Phi_{n} \mid H_{N-1}^{\mathcal{A}} \Phi_{n}\right\rangle<\mathscr{E}_{N-1}^{\mathcal{A}}+\frac{1}{n} \\
\left\|\Phi_{n}\right\|=1
\end{array}\right.
$$

This is possible since $H_{N-1}$ is defined as a Friedrichs extension starting from $\Lambda_{A, V}^{+, N-1} \mathscr{D}_{N-1}$. We further set

$$
\Upsilon_{n}(x):=\int_{\mathbb{R}^{3(N-2)}}\left|\Phi_{n}\left(x, X^{\prime}\right)\right|^{2} d X^{\prime} .
$$

Next, we pick $0<a<\min \left\{m, \triangle_{0}\right\}, r \in(0,1-\varepsilon / 4)$, and $r^{\prime} \in(0,1)$ such that

$$
(1-r) a>(1+r) \tau, \quad s:=r+r^{\prime}-1>0 .
$$

Here $\tau$ appears in (23). We further pick some cut-off function, $\vartheta \in C^{\infty}(\mathbb{R},[0,1])$, such that $\vartheta \equiv 0$ on $(-\infty, s / 2]$ and $\vartheta \equiv 1$ on $[s, \infty)$. By Lemma 3.9] we know that $\left|D_{0}^{(1)}\right|^{1 / 2} \Phi_{n} \in \mathscr{H}_{N-1}$, where the superscript (1) again indicates that the operator acts on the first variable. Therefore, we find a subsequence, $\left\{R_{k_{n}}\right\}_{n \in \mathbb{N}}$, of $\left\{R_{k}\right\}_{k \in \mathbb{N}}$ such that, for every $n \in \mathbb{N}$,

$$
\left.\left.\int_{\mathbb{R}^{3(N-1)}}|| D_{0}^{(1)}\right|^{1 / 2} \vartheta\left(x_{1} / R_{k_{n}}\right) \Phi_{n}(X)\right|^{2} d X<\frac{1}{n},
$$

As a candidate for a Weyl sequence we then try $\left\{\mathcal{A}_{N} \Psi_{n}\right\}_{n \in \mathbb{N}}$, where

$$
\Psi_{n}:=\Phi_{n} \otimes \Lambda_{A, V}^{+} \psi_{k_{n}} \in \Lambda_{A, V}^{+, N} \mathscr{D}_{N}, \quad n \in \mathbb{N} .
$$

To simplify the notation we again write $n$ instead of $k_{n}$ in the following. Finally, we pick some $c>1$ and set

$$
f(t):=(t+c)^{-1 / 2}\left(t-\mathscr{E}_{N-1}^{\mathcal{A}}-\lambda\right), \quad t>-c .
$$

6.3 Lemma. Let the assumptions of Theorem 2.7(i) be fulfilled. If, in the situation described above, $c>1$ is sufficiently large, then $\mathcal{A}_{N} \Psi_{n} \in \mathcal{D}\left(f\left(H_{N}\right)\right)$, for every $n \in \mathbb{N}$, and

$$
\mathrm{w}-\lim _{n \rightarrow \infty} \mathcal{A}_{N} \Psi_{n}=0, \quad \liminf _{n \rightarrow \infty}\left\|\mathcal{A}_{N} \Psi_{n}\right\|>0, \quad \lim _{n \rightarrow \infty}\left\|f\left(H_{N}\right) \mathcal{A}_{N} \Psi_{n}\right\|=0 .
$$

In particular, $\mathscr{E}_{N-1}^{\mathcal{A}}+\lambda \in \sigma_{\mathrm{ess}}\left(H_{N}\right)$. 
Proof: First, suppose that (109) holds true. If $c>1$ is chosen sufficiently large, then $f$ is strictly monotonically increasing on $\sigma\left(H_{N}\right)$. If $I$ is some small open interval around $\mathscr{E}_{N-1}^{\mathcal{A}}+\lambda$ we thus get $E_{I}\left(H_{N}\right)=E_{f(I)}\left(f\left(H_{N}\right)\right)$. By (109) and the Weyl criterion applied to $f\left(H_{N}\right)$ it follows that $\infty=\operatorname{dim} \operatorname{Ran}\left(E_{f(I)}\left(f\left(H_{N}\right)\right)\right)=$ $\operatorname{dim} \operatorname{Ran}\left(E_{I}\left(H_{N}\right)\right)$.

To verify (109) we first notice that $\Psi_{n} \rightarrow 0$, as $n \rightarrow \infty$, because of (22). Exactly as in [30, §4] we can also check that $\lim \inf \left\|\mathcal{A}_{N} \Psi_{n}\right\|>0$. So it suffices to show that $\left\|f\left(H_{N}\right) \Psi_{n}\right\| \rightarrow 0$, as $H_{N}$ commutes with $\mathcal{A}_{N}$. Since $\psi_{n}$ and $\Phi_{n}$ are normalized and $\Psi_{n}=\Phi_{n} \otimes \Lambda_{A, V}^{+} \psi_{n} \in \Lambda_{A, V}^{+, N} \mathscr{D}_{N}$ we obtain

$$
\begin{aligned}
& \left\|f\left(H_{N}\right) \Psi_{n}\right\| \\
& \leqslant \quad\left\|\left(H_{N}+c\right)^{-\frac{1}{2}}\left(H_{N-1}-\mathscr{E}_{N-1}^{\mathcal{A}}\right)^{\frac{1}{2}} \otimes \mathbb{1}_{\mathscr{H}^{+}}\right\|\left\|\left(H_{N-1}-\mathscr{E}_{N-1}^{\mathcal{A}}\right)^{\frac{1}{2}} \Phi_{n}\right\| \\
& \quad+\left\|\left(D_{A, V_{\mathrm{C}}}-\lambda\right) \Lambda_{A, V}^{+} \psi_{n}\right\| \\
& \quad+\sum_{i=1}^{N-1}\left\|\left(H_{N}+c\right)^{-\frac{1}{2}} \Lambda_{A, V}^{+, N} W_{i N}^{\frac{1}{2}}\right\|\left\|W_{i N}^{\frac{1}{2}}\left(\Phi_{n} \otimes \Lambda_{A, V}^{+} \psi_{n}\right)\right\|
\end{aligned}
$$

We first show that the operator norm in (112) is actually finite. In fact,

$$
\left\|\left(H_{N}+c\right)^{-1 / 2} \Lambda_{A, V}^{+, N} W_{i N}^{1 / 2}\right\|=\left\|W_{i N}^{1 / 2} \Lambda_{A, V}^{+, N}\left(H_{N}+c\right)^{-1 / 2}\right\| \leqslant 1,
$$

since $W \geqslant 0, \Lambda_{A, V}^{+} D_{A, V_{\mathrm{C}}} \Lambda_{A, V}^{+} \geqslant-C^{\prime} \Lambda_{A, V}^{+}, H_{N-1} \geqslant \mathscr{E}_{N-1}^{\mathcal{A}} \Lambda_{A, V}^{+, N-1}$, and, hence,

$$
\left\|W_{i N}^{1 / 2} \Lambda_{A, V}^{+, N} \phi\right\|^{2}=\left\langle\phi \mid \Lambda_{A, V}^{+, N} W_{i N} \Lambda_{A, V}^{+, N} \phi\right\rangle \leqslant\left\langle\phi \mid\left(H_{N}+c\right) \phi\right\rangle,
$$

for $\phi \in \Lambda_{A, V}^{+, N} \mathscr{D}_{N}$. Using similar estimates and (104) it is straightforward to check that the term in (110) converges to zero provided $c>1$ is sufficiently large. The norm in (111) tends to zero by Lemma 6.1. The claim now follows from Lemma 6.4 below which implies that the remaining norm in (112) tends to zero, too.

The first inequality of the following lemma is used in the proof of Lemma 6.3 and the second one in Section 7.

6.4 Lemma. There are $\kappa, C \in(0, \infty)$ such that, for all $n \in \mathbb{N}$,

$$
\int_{\mathbb{R}^{6}} W(x, y) \Upsilon_{n}(y)\left|\Lambda_{A, V}^{+} \psi_{n}(x)\right|^{2} d(x, y) \leqslant \sup _{\substack{|x-y| \geqslant \\\left(1-r^{\prime}\right) R_{n}}} W(x, y)+C e^{-\kappa R_{n}}+\frac{1}{n}
$$


If $B$ is bounded, then there is some $C^{\prime} \in(0, \infty)$ such that, for all $n \in \mathbb{N}$,

$$
\begin{aligned}
& \int_{\mathbb{R}^{6}} W(x, y) \Upsilon_{n}(y)\left|\Lambda_{A, V}^{+} \psi_{n}(x)\right|^{2} d(x, y) \\
& \leqslant \sup _{\substack{|x-y| \geqslant \\
(1-\varepsilon) R_{n}}} W(x, y)\left\|\Lambda_{A, V}^{+} \psi_{n}\right\|^{2}+C^{\prime}\left(1+\|B\|_{\infty}\right) e^{-a \varepsilon R_{n} / 2} \\
& \quad+C^{\prime} \int_{\left\{|y| \geqslant \varepsilon R_{n} / 2\right\}} \Upsilon_{n}(y) d y\left(1+\|B\|_{\infty}\right)\left\|\left(D_{A, V}+i\right) \psi_{n}\right\|^{2} .
\end{aligned}
$$

Proof: For $n \in \mathbb{N}$, we pick a weight function, $F_{n} \in C^{\infty}\left(\mathbb{R}^{3},[0, \infty)\right)$, with $F_{n} \equiv 0$ on $\mathbb{R}^{3} \backslash \mathcal{B}_{R_{n}}(0), F_{n} \geqslant(1-r) a R_{n}-a^{\prime}$ on $\mathcal{B}_{r R_{n}}(0)$ and $\left\|\nabla F_{n}\right\|_{\infty} \leqslant a$. Here $a$ and $r$ are the parameters from (106) and $a^{\prime}>0$ is some fixed, $n$-independent constant. Since $\psi_{n}=\chi_{n} \psi_{n}$ and $\mathbb{1}_{\mathcal{B}_{r R_{n}}(0)} \chi_{n}=0$ we obtain

$$
\begin{aligned}
& \int_{\left\{|x-y|<\left(1-r^{\prime}\right) R_{n}\right\}} \mathbb{1}_{\mathcal{B}_{r R_{n}}(0)}(x) W(x, y) \Upsilon_{n}(y)\left|\Lambda_{A, V}^{+} \psi_{n}(x)\right|^{2} d(x, y) \\
& \leqslant\left\|\mathbb{1}_{\mathcal{B}_{r R_{n}}(0)} e^{-F_{n}}\right\|_{\infty} \sup _{|y| \leqslant\left(r+1-r^{\prime}\right) R_{n}}\left\|W_{y}^{1 / 2} e^{F_{n}}\left[\Lambda_{A, V}^{+}, \chi_{n} e^{-F_{n}}\right] \psi_{n}\right\|^{2}\left\|\Upsilon_{n}\right\|_{1} \\
& \leqslant C^{\prime} e^{-(1-r) a R_{n}} \sup _{|y| \leqslant(1+r) R_{n}}(1+|B(y)|) \leqslant C^{\prime \prime} e^{-([1-r] a-[1+r] \tau) R_{n}}
\end{aligned}
$$

In the last two steps we make use of Proposition 3.11 and (23). Next, if $|x-y| \leqslant\left(1-r^{\prime}\right) R_{n}$ and $\mathbb{1}_{\mathcal{B}_{r R_{n}}(0)}(x)=0$, then $|y| \geqslant\left(r+r^{\prime}-1\right) R_{n}=s R_{n}$, and by the choice of $\vartheta$ (see the paragraph below (106)) it follows that

$$
\begin{gathered}
\int_{\left\{|x-y| \leqslant\left(1-r^{\prime}\right) R_{n}\right\}}\left(1-\mathbb{1}_{\mathcal{B}_{r R_{n}}(0)}(x)\right) W(x, y) \Upsilon_{n}(y)\left|\Lambda_{A, V}^{+} \psi_{n}(x)\right|^{2} d(x, y) \\
\leqslant \sup _{|x| \geqslant r R_{n}} \int_{\mathbb{R}^{3}} W(x, y) \vartheta\left(y / R_{n}\right) \Upsilon_{n}(y) d y\left\|\Lambda_{A, V}^{+} \psi_{n}\right\|^{2} .
\end{gathered}
$$

On account of (106), Kato's inequality, and (107)the first asserted estimate follows from (113) and (114). The second one is derived similarly by means of Lemma 3.13 and the replacements $r \mapsto 1-\varepsilon / 2, r^{\prime} \mapsto \varepsilon$. Note that $\mathbb{1}_{\mathcal{B}_{(1+\varepsilon / 2) R_{n}}(0)} \chi_{n}=0$, which is used to derive the analogue of (113).

\section{$7 \quad$ Existence of eigenvalues}

In this section we prove Theorem 2.9 which asserts that $H_{N}^{\mathcal{A}}$ possesses infinitely many eigenvalues below $\inf \sigma_{\text {ess }}\left(H_{N}^{\mathcal{A}}\right)=1+\mathscr{E}_{N-1}^{\mathcal{A}}$. We proceed along the lines 
of [30, §6] with a few changes. In particular, we replace the arguments of [30] that employ explicite position or momentum space representations of $\Lambda_{0}^{+}$ by more abstract ones. Throughout this section we always assume without further notice that the assumptions of Theorem [2.9, i.e., Hypothesis 5, are fulfilled.

Proof of Theorem 2.9: We proceed by induction on $N$ and start with the induction step. So, we pick $N \in \mathbb{N}, N \geqslant 2$, and assume that $H_{N-1}^{\mathcal{A}}$ possesses infinitely many eigenvalues below $\mathscr{E}_{N-2}^{\mathcal{A}}+1$. In particular, we can pick a normalized ground state of $H_{N-1}^{\mathcal{A}}$, which we denote by $\Phi$. Moreover, we denote the transposition operator which flips the $i^{\text {th }}$ and $N^{\text {th }}$ electron variable by $\pi_{i N}$, $1 \leqslant i<N$, and set $\pi_{N N}:=\mathbb{1}$. The vectors $\psi_{1}, \psi_{2}, \ldots$ are the elements of the sequence appearing in Hypothesis 5 .

Now, let $d \in \mathbb{N}$. By Lemma 7.7 below we know that, for all sufficiently large $m_{0} \in \mathbb{N}$, the set $\left\{\mathcal{A}_{N}\left(\Phi \otimes \Lambda_{A, V}^{+} \psi_{n}\right)\right\}_{n=m_{0}}^{m_{0}+d}$, where

$$
\mathcal{A}_{N}\left(\Phi \otimes \Lambda_{A, V}^{+} \psi_{n}\right)=\frac{1}{N} \sum_{i=1}^{N}(-1)^{N-i} \pi_{i N}\left(\Phi \otimes \Lambda_{A, V}^{+} \psi_{n}\right), \quad n \in \mathbb{N},
$$

is linearly independent. Our goal then is to show that the expectation of

$$
\widehat{H}_{N}:=H_{N}-\mathscr{E}_{N-1}^{\mathcal{A}}-1
$$

with respect to any linear combination of the vectors $\left\{\mathcal{A}_{N}\left(\Phi \otimes \Lambda_{A, V}^{+} \psi_{n}\right)\right\}_{n=m_{0}}^{m_{0}+d}$ is strictly negative provided $m_{0} \in \mathbb{N}$ is large enough. Since $d$ is arbitrary the assertion of Theorem 2.9 then follows from the minimax principle. For $c_{m_{0}}, \ldots, c_{m_{0}+d} \in \mathbb{C}$, and

$$
\Psi:=\sum_{n=m_{0}}^{m_{0}+d} c_{n} \sum_{i=1}^{N} \frac{(-1)^{N-i}}{N^{1 / 2}} \pi_{i N}\left(\Phi \otimes \Lambda_{A, V}^{+} \psi_{n}\right),
$$

we obtain as in [30] by means of the anti-symmetry of $\Phi$,

$$
\begin{aligned}
\left\langle\Psi \mid \widehat{H}_{N} \Psi\right\rangle & \leqslant \sum_{n=m_{0}}^{m_{0}+d}\left|c_{n}\right|^{2}\left\langle\Phi \otimes \Lambda_{A, V}^{+} \psi_{n} \mid \widehat{H}_{N}\left(\Phi \otimes \Lambda_{A, V}^{+} \psi_{n}\right)\right\rangle \\
+(N-1) & \sum_{n, m=m_{0}}^{m_{0}+d}\left|c_{n}\right|\left|c_{m}\right|\left|\left\langle\pi_{1 N}\left(\Phi \otimes \Lambda_{A, V}^{+} \psi_{n}\right) \mid \widehat{H}_{N}\left(\Phi \otimes \Lambda_{A, V}^{+} \psi_{m}\right)\right\rangle\right| \\
& +\sum_{\substack{n, m=m_{0} \\
m \neq n}}^{m_{0}+d}\left|c_{n}\right|\left|c_{m}\right|\left|\left\langle\Phi \otimes \Lambda_{A, V}^{+} \psi_{n} \mid \widehat{H}_{N}\left(\Phi \otimes \Lambda_{A, V}^{+} \psi_{m}\right)\right\rangle\right| .
\end{aligned}
$$


Combining the eigenvalue equation $\left(H_{N-1}-\mathscr{E}_{N-1}^{\mathcal{A}}\right) \Phi=0$ with Lemmata 7.1 7.4. Hypothesis 5, and (103), we find some $\delta_{0}>0$ such that the scalar product in (116) is bounded from above by $-\delta_{0} R_{m_{0}}^{-1}+o\left(R_{m_{0}}^{-1}\right)$, as $m_{0}$ gets large. Here the numbers $R_{1}, R_{2}, \ldots$ are those appearing in Hypothesis 5, Lemmata 7.5 and 7.6 imply that the scalar products in (117) and (118) are of order $\mathcal{O}\left(R_{m_{0}}^{-K}\right)$, as $m_{0} \rightarrow \infty$, for every $K \in \mathbb{N}$. By the Cauchy-Schwarz inequality we find some $\delta_{0}^{\prime}>0$ such that

$$
\left\langle\Psi \mid \widehat{H}_{N} \Psi\right\rangle \leqslant-\delta_{0}^{\prime} \sum_{n=m_{0}}^{m_{0}+d}\left|c_{n}\right|^{2},
$$

for all $c_{m_{0}}, \ldots, c_{m_{0}+d} \in \mathbb{C}$, if $m_{0}$ is sufficiently large (depending on $d$ ). This concludes the induction step.

Finally, the case $N=1$ is treated in the same way as the induction step $N \rightarrow N+1$ (setting $\mathscr{E}_{0}^{\mathcal{A}}:=0$ and ignoring $\Phi, W$, and the term (117)) .

To show that the contribution coming from the (one-particle) kinetic energy of $\psi_{n}$ decreases faster than its negative potential energy we make use of the requirement that the $\psi_{n}$ have vanishing lower spinor components, $\psi_{n}=$ $\left(\psi_{n, 1}, \psi_{n, 2}, 0,0\right)^{\top}, n \in \mathbb{N}$. This has also been used in [30] together with explicite formulas for $\Lambda_{0}^{+}$. We replace these arguments by the following observation:

7.1 Lemma. There is some $C \in(0, \infty)$ such that

$$
0 \leqslant\left\langle\Lambda_{A}^{+} \psi_{n} \mid\left(D_{A}-1\right) \Lambda_{A}^{+} \psi_{n}\right\rangle \leqslant C R_{n}^{-2}, \quad n \in \mathbb{N} .
$$

Proof: Since the last two components of $\psi_{n}$ are zero we have $(\beta-\mathbb{1}) \psi_{n}=0$. If we denote the projection onto the first two spinor components, $L^{2}\left(\mathbb{R}^{3}, \mathbb{C}^{4}\right) \ni$ $\left(\varphi_{1}, \varphi_{2}, \varphi_{3}, \varphi_{4}\right)^{\top} \mapsto\left(\varphi_{1}, \varphi_{2}, 0,0\right)^{\top}$, by $p$ then we also have $p \alpha_{i} \psi_{n}=0=$ $p \alpha_{i} \partial_{i} \psi_{n}, i=1,2,3$, and, therefore, $p\left(D_{A}-1\right) \psi_{n}=0$. Moreover, $\left[p, D_{A}^{2}\right]=0$ and, hence, $\left[p,\left|D_{A}\right|^{-1}\right]=0$. This implies

$$
\begin{aligned}
& \left|\left\langle\Lambda_{A}^{+} \psi_{n} \mid\left(D_{A}-1\right) \Lambda_{A}^{+} \psi_{n}\right\rangle\right| \\
& =\left|\frac{1}{2}\left\langle\psi_{n} \mid p\left(D_{A}-1\right) \psi_{n}\right\rangle+\frac{1}{2}\left\langle\operatorname{sgn}\left(D_{A}\right) \psi_{n} \mid\left(D_{A}-1\right) \psi_{n}\right\rangle\right| \\
& =\mid \frac{1}{2}\left\langle\left.\left(D_{A}-1\right) \psi_{n}|| D_{A}\right|^{-1}\left(D_{A}-1\right) \psi_{n}\right\rangle \\
& \quad+\frac{1}{2}\left\langle\left.\psi_{n}|| D_{A}\right|^{-1} p\left(D_{A}-1\right) \psi_{n}\right\rangle \mid \\
& \leqslant \frac{1}{2}\left\|\left(D_{A}-1\right) \psi_{n}\right\|^{2}=\mathcal{O}\left(1 / R_{n}^{2}\right) .
\end{aligned}
$$

In the last step we apply Hypothesis 5 . 
In the following we split $V_{\mathrm{C}}$ into a singular and regular part, $V_{\mathrm{C}}=V_{\mathrm{C}}^{\mathrm{s}}+V_{\mathrm{C}}^{\mathrm{r}}$, where $V_{\mathrm{C}}^{\mathrm{s}}$ is defined in (28). By Hypothesis $1 V_{\mathrm{C}}^{\mathrm{r}}$ is bounded.

7.2 Lemma. As $n$ tends to infinity,

$$
\begin{aligned}
& \left\langle\Lambda_{A, V}^{+} \psi_{n} \mid\left(D_{A, V_{\mathrm{C}}}-1\right) \Lambda_{A, V}^{+} \psi_{n}\right\rangle \\
& \quad=\left\langle\Lambda_{A, V}^{+} \psi_{n} \mid V_{\mathrm{C}}^{\mathrm{r}} \Lambda_{A, V}^{+} \psi_{n}\right\rangle+\left\langle\Lambda_{A}^{+} \psi_{n} \mid\left(D_{A}-1\right) \Lambda_{A}^{+} \psi_{n}\right\rangle+o\left(R_{n}^{-1}\right) .
\end{aligned}
$$

Proof: We let $\chi_{n}, n \in \mathbb{N}$, denote the cut-off functions introduced in the paragraph preceeding Lemma 6.1. Then the assertion follows from Corollary 3.15 applied to $D_{A, V_{\mathrm{C}}^{\mathrm{s}}}-1$ with $\zeta=\chi_{n}$, since by Lemma 7.1 and Hypothesis 2 ,

$$
\left(\left\|\chi_{n} V\right\|+\left\|\nabla \chi_{n}\right\|\right) \sqrt{\left\langle\Lambda_{A}^{+} \psi_{n} \mid\left(D_{A}-1\right) \Lambda_{A}^{+} \psi_{n}\right\rangle\left\|\psi_{n}\right\|^{2}}=o\left(R_{n}^{-1}\right) .
$$

In the next lemma we single out the leading order negative contribution to (116).

7.3 Lemma. There is some constant $C \in(0, \infty)$ such that, for all sufficiently large $n \in \mathbb{N}$,

$$
\left\langle\Lambda_{A, V}^{+} \psi_{n} \mid V_{\mathrm{C}}^{\mathrm{r}} \Lambda_{A, V}^{+} \psi_{n}\right\rangle \leqslant v_{\star}\left(\delta, R_{n}\right)\left\|\Lambda_{A, V}^{+} \psi_{n}\right\|^{2}+C e^{-R_{n} / C},
$$

where $v_{\star}\left(\delta, R_{n}\right)$ is given by (25) .

Proof: We pick some even function $f \in C^{\infty}(\mathbb{R},[0, \infty))$ such that $f \equiv 1$ on $[\delta, \infty), f \equiv 0$ on $[0, \delta / 2]$, and $\left|f^{\prime}\right| \leqslant 4 / \delta$. (Recall (26).) For some $a \in$ $\left(0, \delta \min \left\{\triangle_{0}, m\right\} / 4\right)$, we define exponential weights, $F_{n}(x):=a R_{n} f\left(|x| / R_{n}\right)$, $n \in \mathbb{N}$. Using the notation introduced in (24) \&(25) we then obtain, for all sufficiently large $n \in \mathbb{N}$,

$$
\begin{aligned}
\left\langle\Lambda_{A, V}^{+} \psi_{n} \mid V_{\mathrm{C}}^{\mathrm{r}} \Lambda_{A, V}^{+} \psi_{n}\right\rangle \leqslant & \left\langle\Lambda_{A, V}^{+} \psi_{n} \mid \mathbb{1}_{S_{\delta}\left(R_{n}\right)} V_{\mathrm{C}}^{\mathrm{r}} \Lambda_{A, V}^{+} \psi_{n}\right\rangle \\
& +\left\|V_{\mathrm{C}}^{\mathrm{r}}\right\|\left\|\mathbb{1}_{\mathbb{R}^{3} \backslash S_{\delta}\left(R_{n}\right)} e^{-F_{n}}\right\|\left\|e^{F_{n}} \Lambda_{A, V}^{+} e^{-F_{n}}\right\| .
\end{aligned}
$$

where, by (25) and Pythagoras' theorem,

$$
\begin{aligned}
& \left\langle\Lambda_{A, V}^{+} \psi_{n} \mid \mathbb{1}_{S_{\delta}\left(R_{n}\right)} V_{\mathrm{C}}^{\mathrm{r}} \Lambda_{A, V}^{+} \psi_{n}\right\rangle \\
& \quad \leqslant v_{\star}\left(\delta, R_{n}\right)\left(\left\|\Lambda_{A, V}^{+} \psi_{n}\right\|^{2}-\left\|\mathbb{1}_{\mathbb{R}^{3} \backslash S_{\delta}\left(R_{n}\right)} \Lambda_{A, V}^{+} \psi_{n}\right\|^{2}\right) \\
& \quad \leqslant v_{\star}\left(\delta, R_{n}\right)\left\|\Lambda_{A, V}^{+} \psi_{n}\right\|^{2}+\left|v_{\star}\left(\delta, R_{n}\right)\right|\left\|\mathbb{1}_{\mathbb{R}^{3} \backslash S_{\delta}\left(R_{n}\right)} e^{-F_{n}}\right\|^{2}\left\|e^{F_{n}} \Lambda_{A, V}^{+} e^{-F_{n}}\right\|^{2} .
\end{aligned}
$$

By (24), (26) , and the choice of $F_{n}$ we know that $\left\|\mathbb{1}_{\mathbb{R}^{3} \backslash S_{\delta}\left(R_{n}\right)} e^{-F_{n}}\right\| \leqslant C e^{-a \delta R_{n} / 2}$, which implies the assertion of the lemma. 
From now on, we always assume that the induction hypothesis made in the proof of Theorem 2.9 is fulfilled and that $\Phi$ is a normalized ground state eigenvector of $H_{N-1}^{\mathcal{A}}$. So, $H_{N-1}^{\mathcal{A}} \Phi=\mathscr{E}_{N-1}^{\mathcal{A}} \Phi, \mathscr{E}_{N-1}^{\mathcal{A}}<\mathscr{E}_{N-2}^{\mathcal{A}}+1$. Given $\delta \in\left(0, \frac{1}{N}\right)$ we pick some $\varepsilon \in(0,1)$ as in Hypothesis 5 (i). Then the following assertion is valid:

7.4 Lemma. As $n$ tends to infinity, we have, for $1 \leqslant i \leqslant N-1$,

$$
\left\langle\Phi \otimes \Lambda_{A, V}^{+} \psi_{n} \mid W_{i N} \Phi \otimes \Lambda_{A, V}^{+} \psi_{n}\right\rangle \leqslant \sup _{\substack{|x-y| \geqslant \\(1-\varepsilon) R_{n}}} W(x, y)\left\|\Lambda_{A, V}^{+} \psi_{n}\right\|^{2}+\mathcal{O}\left(R_{n}^{-\infty}\right) .
$$

Proof: This follows from Lemma 6.4 with $\Upsilon_{n}(y)=\int_{\mathbb{R}^{3(N-2)}}\left|\Phi\left(y, X^{\prime}\right)\right|^{2} d X^{\prime}$ and the exponential decay of $\Phi$, which is ensured by Theorem 2.4 and the induction hypothesis.

Now, we turn to the discussion of the terms in (117).

7.5 Lemma. As $n$ and $m$ tend to infinity,

$$
\sigma_{n m}:=\left|\left\langle\pi_{1 N}\left(\Phi \otimes \Lambda_{A, V}^{+} \psi_{n}\right) \mid \widehat{H}_{N}\left(\Phi \otimes \Lambda_{A, V}^{+} \psi_{m}\right)\right\rangle\right|=\mathcal{O}\left(R_{\min \{n, m\}}^{-\infty}\right) .
$$

Proof: We pick $\chi \in C_{0}^{\infty}\left(\mathbb{R}^{3},[0,1]\right)$ such that $\chi \equiv 1$ on $\mathcal{B}_{1 / 4}(0)$ and $\chi \equiv 0$ outside $\mathcal{B}_{1 / 2}(0)$ and set $\chi_{n}:=\chi\left(\cdot / R_{n}\right)$ and $\bar{\chi}_{n}:=1-\chi_{n}$, for $n \in \mathbb{N}$. As in [30] we find

$$
\begin{aligned}
\sigma_{n m} \leqslant & \left|\left\langle\left\{\chi_{n} \Lambda_{A, V}^{+} \psi_{n}\right\} \otimes \Phi \mid \Phi \otimes\left\{\left(D_{A, V_{\mathrm{C}}}-1\right) \Lambda_{A, V}^{+} \psi_{m}\right\}\right\rangle\right| \\
& +\left|\left\langle\left\{\Lambda_{A, V}^{+} \psi_{n}\right\} \otimes \Phi \mid\left\{\bar{\chi}_{n}^{(1)} \Phi\right\} \otimes\left\{\left(D_{A, V_{\mathrm{C}}}-1\right) \Lambda_{A, V}^{+} \psi_{m}\right\}\right\rangle\right| \\
& +\sum_{i=1}^{N-1}\left|\left\langle\left\{\chi_{n} \Lambda_{A, V}^{+} \psi_{n}\right\} \otimes \Phi \mid W_{i N} \Phi \otimes\left(\Lambda_{A, V}^{+} \psi_{m}\right)\right\rangle\right| \\
& +\sum_{i=1}^{N-1}\left|\left\langle\left\{\Lambda_{A, V}^{+} \psi_{n}\right\} \otimes \Phi \mid W_{i N}\left(\bar{\chi}_{n}^{(1)} \Phi\right) \otimes\left(\Lambda_{A, V}^{+} \psi_{m}\right)\right\rangle\right| \\
=: & Y_{1}+Y_{2}+\sum_{i=1}^{N-1} Y_{3 i}+\sum_{i=1}^{N-1} Y_{4 i} .
\end{aligned}
$$

For the first two summands we find

$$
Y_{1}+Y_{2} \leqslant\left\|\left(D_{A, V_{\mathrm{C}}}-1\right) \Lambda_{A, V}^{+} \psi_{m}\right\|\left(\left\|\chi_{n} \Lambda_{A, V}^{+} \psi_{n}\right\|+\left\|\bar{\chi}_{n}^{(1)} \Phi\right\|\right),
$$

where the right side is of order $\mathcal{O}\left(R_{\min \{n, m\}}^{-\infty}\right)$ due to the exponential localization of $\Phi$ and the support properties of $\psi_{n}$ and $\chi_{n}$. Moreover, we observe that, for 
$i=2, \ldots, N-1$

$$
\begin{aligned}
& Y_{3 i} \leqslant\left\|\chi_{n} \Lambda_{A, V}^{+} \psi_{n}\right\|\left\|W_{i N}^{1 / 2} \Phi\right\|\|\Phi\| \sup _{y \in \mathbb{R}^{3}}\left\|W_{y}^{1 / 2} \Lambda_{A, V}^{+} \psi_{m}\right\| \\
& Y_{4 i} \leqslant\left\|\Lambda_{A, V}^{+} \psi_{n}\right\|\left\|W_{i N}^{1 / 2} \Phi\right\|\left\|\bar{\chi}_{n}^{(1)} \Phi\right\| \sup _{y \in \mathbb{R}^{3}}\left\|W_{y}^{1 / 2} \Lambda_{A, V}^{+} \psi_{m}\right\|
\end{aligned}
$$

Here the norms $\left\|W_{i N}^{1 / 2} \Phi\right\|, i=2, \ldots, N-1$, are actually finite since $\Phi \in$ $\operatorname{Ker}\left(H_{N-1}-\mathscr{E}_{N-1}^{\mathcal{A}}\right)$ implies

$$
\left\|W_{i N}^{1 / 2} \Phi\right\|^{2}=\left\langle\Phi \mid \Lambda_{A, V}^{+, N-1} W_{i N} \Lambda_{A, V}^{+, N-1} \Phi\right\rangle \leqslant\left(\mathscr{E}_{N-1}^{\mathcal{A}}+C\right)\|\Phi\|^{2},
$$

for some constant $C \in(0, \infty)$. Finally,

$$
\begin{aligned}
& Y_{31} \leqslant \sup _{y \in \mathbb{R}^{3}}\left\|W_{y}^{1 / 2} \chi_{n} \Lambda_{A, V}^{+} \psi_{n}\right\|\|\Phi\|^{2} \sup _{y \in \mathbb{R}^{3}}\left\|W_{y}^{1 / 2} \Lambda_{A, V}^{+} \psi_{m}\right\|, \\
& Y_{41} \leqslant \sup _{y \in \mathbb{R}^{3}}\left\|W_{y}^{1 / 2} \Lambda_{A, V}^{+} \psi_{n}\right\|\|\Phi\|\left\|\bar{\chi}_{n}^{(1)} \Phi\right\| \sup _{y \in \mathbb{R}^{3}}\left\|W_{y}^{1 / 2} \Lambda_{A, V}^{+} \psi_{m}\right\| .
\end{aligned}
$$

We pick $f \in C^{\infty}(\mathbb{R},[0, \infty))$ such that $f \equiv 0$ on $[1, \infty), f \equiv 1$ on $(-\infty, 1 / 2]$, and $-3 \leqslant f^{\prime} \leqslant 0$, and set $F_{n}(x)=a R_{n} f\left(|x| / R_{n}\right), x \in \mathbb{R}^{3}, n \in \mathbb{N}$, where $a \in\left(0, \min \left\{\triangle_{0}, m\right\} / 3\right)$. Since $\chi_{n} \psi_{n}=0$, we find

$$
\begin{aligned}
& \sup _{y \in \mathbb{R}^{3}}\left\|W_{y}^{1 / 2} \chi_{n} \Lambda_{A, V}^{+} \psi_{n}\right\| \\
& \quad \leqslant \sup _{|x| \leqslant R_{n} / 2} e^{-F_{n}} \sup _{y \in \mathbb{R}^{3}}\left\|W_{y}^{1 / 2}\left[\Lambda_{A, V}^{+}, \chi_{n} e^{F_{n}}\right] e^{-F_{n}}\right\|\left\|\psi_{n}\right\| .
\end{aligned}
$$

This estimate, the exponential decay of $\Phi$, and Lemma 3.13 imply that the terms $Y_{3 i}$ and $Y_{4 i}, 1 \leqslant i \leqslant N-1$, vanish of order $\mathcal{O}\left(R_{\min \{n, m\}}^{-\infty}\right)$ also.

Finally, we discuss the terms in (118).

7.6 Lemma. As $n$ tends to infinity, it holds, for all $m>n$,

$$
\left|\left\langle\Phi \otimes \Lambda_{A, V}^{+} \psi_{n} \mid \widehat{H}_{N}\left(\Phi \otimes \Lambda_{A, V}^{+} \psi_{m}\right)\right\rangle\right|=\mathcal{O}\left(R_{n}^{-\infty}\right)
$$

Proof: We pick a family of smooth weight functions, $\left\{F_{k \ell}\right\}_{k, \ell \in \mathbb{N}}$, such that $F_{k \ell} \equiv 0$ on $\operatorname{supp}\left(\psi_{k}\right), F_{k \ell}$ is constant outside some ball containing $\operatorname{supp}\left(\psi_{k}\right)$ and $\operatorname{supp}\left(\psi_{\ell}\right),\left\|\nabla F_{k \ell}\right\|_{\infty} \leqslant a<1$, and

$$
g_{k \ell}:=\left\|e^{-F_{k \ell}-F_{\ell k}}\right\|_{\infty} \leqslant C e^{-a^{\prime} \min \left\{R_{k}, R_{\ell}\right\}}, \quad k, \ell \in \mathbb{N},
$$


where $a, a^{\prime} \in(0,1)$ and $C \in(0, \infty)$ do not depend on $k, \ell \in \mathbb{N}$. In view of $(26)$ it is easy to see that such a family exists. Then we observe that

$$
\begin{aligned}
& \left|\left\langle\Phi \otimes \Lambda_{A, V}^{+} \psi_{n} \mid \widehat{H}_{N}\left(\Phi \otimes \Lambda_{A, V}^{+} \psi_{m}\right)\right\rangle\right| \\
& \leqslant\left|\left\langle\Lambda_{A, V}^{+} \psi_{n} \mid\left(D_{A, V}-1\right) \psi_{m}\right\rangle\right|+\left|\left\langle\Lambda_{A, V}^{+} \psi_{n} \mid\left(V_{\mathrm{H}}+V_{\mathrm{E}}\right) \Lambda_{A, V}^{+} \psi_{m}\right\rangle\right| \\
& \quad+\sum_{1 \leqslant i<N}\left|\left\langle W_{i N}^{1 / 2} \Phi \otimes \Lambda_{A, V}^{+} \psi_{n} \mid W_{i N}^{1 / 2} \Phi \otimes \Lambda_{A, V}^{+} \psi_{m}\right\rangle\right| \\
& \leqslant g_{n m}\left\|e^{F_{n m}} \Lambda_{A, V}^{+} e^{-F_{n m}}\right\|\left\|\psi_{n}\right\|\left\{\left\|\left(D_{A, V}-1\right) \psi_{m}\right\|\right. \\
& \left.\quad+\left\|\left(i \alpha \cdot \nabla F_{m n}+V_{\mathrm{H}}+\left[e^{F_{m n}}, V_{\mathrm{E}}\right] e^{-F_{m n}}\right) \psi_{m}\right\|\right\} \\
& +g_{n m}\left\|e^{F_{m n}}\left(V_{\mathrm{H}}+V_{\mathrm{E}}\right) e^{-F_{m n}}\right\| \sup _{k \neq \ell}\left\|e^{F_{k \ell}} \Lambda_{A, V}^{+} e^{-F_{k \ell}} \psi_{k}\right\|^{2} \\
& +g_{n m}(N-1) \sup _{k \neq \ell} \sup _{y \in \mathbb{R}^{3}}\left\|W_{y}^{1 / 2} e^{F_{k \ell}} \Lambda_{A, V}^{+} e^{-F_{k \ell}} \psi_{k}\right\|^{2} .
\end{aligned}
$$

By virtue of Proposition 3.11 and Lemma 3.13 we know that all terms behind the factors $g_{n m}$ appearing here are uniformly bounded which shows that the assertion holds true.

Applying the above arguments in an easier situation we obtain the following lemma.

7.7 Lemma. For every $d \in \mathbb{N}$, there is some $n_{0} \in \mathbb{N}$ such that the set of vectors $\left\{\mathcal{A}_{N}\left(\Phi \otimes \Lambda_{A, V}^{+} \psi_{n}\right)\right\}_{n=m_{0}}^{m_{0}+d}$ is linearly independent, for all $m_{0} \in \mathbb{N}, m_{0} \geqslant n_{0}$.

Proof: We pick $\Psi$ as in (115) and estimate $\|\Psi\|^{2}$ from below by an obvious analogue of (116)-(118) with $\widehat{H}_{N}$ replaced by the identity. Now, by virtue of Lemma 6.2 there is some $m_{1} \in \mathbb{N}$ such that $\left\|\Phi \otimes \Lambda_{A, V}^{+} \psi_{n}\right\|=\left\|\Lambda_{A, V}^{+} \psi_{n}\right\| \geqslant 1 / 2$, for all $n \geqslant m_{1}$. The proof of Lemma 7.5 shows that

$$
\left|\left\langle\pi_{1 N}\left(\Phi \otimes \Lambda_{A, V}^{+} \psi_{n}\right) \mid \Phi \otimes \Lambda_{A, V}^{+} \psi_{m}\right\rangle\right|=\mathcal{O}\left(R_{\min \{n, m\}}^{-\infty}\right) .
$$

Furthermore, by employing the exponential weights from the proof of Lemma 7.6 we see that

$$
\left|\left\langle\Phi \otimes \Lambda_{A, V}^{+} \psi_{n} \mid \Phi \otimes \Lambda_{A, V}^{+} \psi_{m}\right\rangle\right|=\left|\left\langle\psi_{n} \mid \Lambda_{A, V}^{+} \psi_{m}\right\rangle\right| \leqslant C e^{-\min \left\{R_{n}, R_{m}\right\} / C} .
$$

Altogether we find some $C^{\prime} \in(0, \infty)$ such that, for $d \in \mathbb{N}$ and all sufficiently large $m_{0} \in \mathbb{N}$,

$$
\|\Psi\|^{2} \geqslant \frac{1}{2} \sum_{n=m_{0}}^{m_{0}+d}\left|c_{n}\right|^{2}-\frac{C^{\prime}(N-1)}{R_{m_{0}}} \sum_{n, m=m_{0}}^{m_{0}+d}\left|c_{n}\right|\left|c_{m}\right|-\frac{C^{\prime}}{R_{m_{0}}} \sum_{\substack{n, m=m_{0} \\ n \neq m}}^{m_{0}+d}\left|c_{n}\right|\left|c_{m}\right| .
$$

Hence, the Cauchy-Schwarz inequality implies that, for sufficiently large $m_{0}, \Psi$ in (115) is zero if and only if $c_{m_{0}}=\ldots=c_{m_{0}+d}=0$. 


\section{A Proofs of Proposition 3.11 and Lemma 3.13}

First, we recall a usefull resolvent identity. We remind the reader that $V_{\mathrm{C}}^{\mathrm{s}}=$ $S\left|V_{\mathrm{C}}^{\mathrm{s}}\right|$ denotes the polar decomposition of the potential defined in (28) and set

$$
M(z):=\left|V_{\mathrm{C}}^{\mathrm{s}}\right|^{1 / 2} R_{0}(z)\left|V_{\mathrm{C}}^{\mathrm{s}}\right|^{1 / 2}, \quad z \in \varrho\left(D_{0}\right) .
$$

A.1 Lemma. ([25, 31, 32]) Assume that $V_{\mathrm{C}}$ fulfills Hypothesis 1 and let $V_{\mathrm{C}}^{\mathrm{s}}$ be given by (28). Then there exist $\eta_{0}>0$ and $\gamma_{0} \in(\gamma, 1)$ such that, for every $\eta \in \mathbb{R} \backslash\left(-\eta_{0}, \eta_{0}\right)$, we have $\|M(i \eta)\| \leqslant \gamma_{0}$ and

$$
R_{0, V_{\mathrm{C}}^{\mathrm{s}}}(i \eta)=R_{0}(i \eta)-\overline{R_{0}(i \eta)\left|V_{\mathrm{C}}^{\mathrm{s}}\right|^{1 / 2}}(1+S \overline{M(i \eta)})^{-1} S\left|V_{\mathrm{C}}^{\mathrm{s}}\right|^{1 / 2} R_{0}(i \eta) .
$$

Notes: The inequality $\left\||\cdot|^{-1 / 2} R_{0}(i \eta)|\cdot|^{-1 / 2}\right\| \leqslant 1$ has been conjectured in [31] and proved in [25]. By means of this inequality and the arguments of [32, Pages $2 \& 3$ (with $k_{s} \equiv 1$ )] we find some $\gamma_{0} \in(\gamma, 1)$ such that $\|M(i \eta)\| \leqslant \gamma_{0}$ provided $|\eta|$ is large enough. The resolvent formula (120) then follows from [31, Lemma 2.2 \& Theorem 2.2].

Proof of Proposition 3.11: We pick some $\zeta \in C_{0}^{\infty}\left(\mathbb{R}^{3},[0,1]\right)$ such that $\zeta=1$ in a neighbourhood of $\mathcal{Y}$ and $\operatorname{supp}(\zeta) \Subset \mathcal{N}$. We set $\bar{\zeta}:=1-\zeta$. Moreover, we pick some $\varrho \in C_{0}^{\infty}\left(\mathbb{R}^{3},[0,1]\right)$ with $\varrho \equiv 1$ on $\mathcal{B}_{1 / 2}(0)$ and $\operatorname{supp}(\varrho) \subset \mathcal{B}_{1}(0)$ and set $\varrho_{y}(x):=\varrho(x-y), x \in \mathbb{R}^{3}$. On account of Proposition 3.6 it suffices to consider

$$
\begin{aligned}
& \left\langle\varrho_{y} W_{y}^{1 / 2} \phi \mid\left[\Lambda_{A, V}^{+}, e^{F} \chi\right] e^{-F} \psi\right\rangle \\
& =\int_{\Gamma}\left\langle\varrho_{y} W_{y}^{1 / 2} \phi \mid \zeta T(z) \psi\right\rangle \frac{d z}{2 \pi}+\int_{\Gamma}\left\langle\varrho_{y} W_{y}^{1 / 2} \phi \mid \bar{\zeta} T(z) \psi\right\rangle \frac{d z}{2 \pi},(12
\end{aligned}
$$

for $\phi \in H^{1 / 2}\left(\mathbb{R}^{3}, \mathbb{C}^{4}\right)$ and $\psi \in \mathscr{H}$, where, by (46) and (55),

$$
\begin{aligned}
T(z) & :=R_{A, V}(z) \widetilde{T} e^{F} R_{A, V}(z) e^{-F}, \quad z \in \Gamma, \\
\widetilde{T} & :=i \alpha \cdot(\chi \nabla F+\nabla \chi)+\left[e^{F} \chi, V_{\mathrm{E}}\right] e^{-F}=\mathcal{O}\left(\|\nabla \chi\|_{\infty}+a\right) .
\end{aligned}
$$

To study the first integral in (121) we write, using (33),

$$
\begin{aligned}
\zeta R_{A, V}(z)= & \zeta R_{0, V_{\mathrm{C}}^{\mathrm{s}}}(z)+R_{0, V_{\mathrm{C}}^{\mathrm{s}}}(z) i \alpha \cdot \nabla \zeta\left(R_{0, V_{\mathrm{C}}^{\mathrm{s}}}(z)-R_{A, V}(z)\right) \\
& -R_{0, V_{\mathrm{C}}^{\mathrm{s}}}(z) \zeta\left\{V-V_{\mathrm{C}}^{\mathrm{s}}+\alpha \cdot A\right\} R_{A, V}(z),
\end{aligned}
$$

where $V_{\mathrm{C}}^{\mathrm{s}}=S\left|V_{\mathrm{C}}^{\mathrm{s}}\right|$ is defined in (28) . Since $\mathcal{D}\left(D_{0, V_{\mathrm{C}}^{\mathrm{s}}}\right) \subset H^{1 / 2}\left(\mathbb{R}^{3}, \mathbb{C}^{4}\right)$ due to Lemma $3.2(\mathrm{e})$ we find $C, C^{\prime} \in(0, \infty)$ such that, for all $y \in \mathbb{R}^{3}$ and all $z \in \Gamma$,

$$
\left\|W_{y}^{1 / 2} R_{0, V_{\mathrm{C}}^{\mathrm{s}}}(z)\right\| \leqslant C\left\|\left|D_{0}\right|^{1 / 2} R_{0, V_{\mathrm{C}}^{\mathrm{s}}}(z)\right\| \leqslant C^{\prime} .
$$


By definition of $T(z)$ and $\widetilde{T}$ and by (38) we thus have

$$
\begin{array}{r}
\int_{\Gamma}\left|\left\langle\varrho_{y} W_{y}^{1 / 2} \phi \mid \zeta\left\{T(z)-R_{0, V_{\mathrm{C}}^{\mathrm{s}}}(z) \widetilde{T} e^{F} R_{A, V}(z) e^{-F}\right\} \psi\right\rangle\right| \frac{|d z|}{2 \pi} \\
\leqslant C_{a, \zeta}\left(a+\|\nabla \chi\|_{\infty}\right) \int_{\mathbb{R}} \frac{d \eta}{1+\eta^{2}}\|\phi\|\|\psi\| .
\end{array}
$$

To treat the remaining part of the first integral in (121) we employ the first resolvent formula and (120) to obtain, for $\eta \in \mathbb{R}$ with $|\eta| \geqslant \eta_{0}$ and $z=e_{0}+i \eta$ ( $\eta_{0}$ is the parameter appearing in Lemma A.1),

$$
\begin{aligned}
& R_{0, V_{\mathrm{C}}^{\mathrm{s}}}(z)=R_{0}(i \eta)+e_{0} R_{0, V_{\mathrm{C}}^{\mathrm{s}}}(z) R_{0, V_{\mathrm{C}}^{\mathrm{s}}}(i \eta) \\
& \quad-\overline{R_{0}(i \eta)\left|V_{\mathrm{C}}^{\mathrm{s}}\right|^{1 / 2}}(1+S \overline{M(i \eta)})^{-1} S\left|V_{\mathrm{C}}^{\mathrm{s}}\right|^{1 / 2}\left|D_{0}\right|^{-1 / 2}\left|D_{0}\right|^{1 / 2} R_{0}(i \eta) .
\end{aligned}
$$

Here the operator $(1+S \overline{M(i \eta)})^{-1}$ is uniformly bounded for $|\eta| \geqslant \eta_{0}$. Moreover,

$$
\left\|W_{y}^{1 / 2} \overline{R_{0}(i \eta)\left|V_{\mathrm{C}}^{\mathrm{s}}\right|^{1 / 2}}\right\| \leqslant C,
$$

uniformly in $y \in \mathbb{R}^{3}$ and $\eta \in \mathbb{R}$, which is a simple consequence of Kato's inequality. In view of (125), (126), and (150) it is therefore clear that it suffices to discuss the contribution coming from the bare resolvent $R_{0}(i \eta)$ in (126). On account of (38), (49), and (123) we find by means of the Cauchy-Schwarz inequality,

$$
\begin{aligned}
& \int_{|\eta| \geqslant \eta_{0}}\left|\left\langle R_{0}(-i \eta) W_{y}^{1 / 2} \varrho_{y} \zeta \phi \mid \widetilde{T} e^{F} R_{A, V}\left(e_{0}+i \eta\right) e^{-F} \psi\right\rangle\right| \frac{d \eta}{2 \pi} \\
& \quad \leqslant\|\widetilde{T}\|\left\|\left(\left|D_{0}\right|^{-1 / 2} W_{y}^{1 / 2}\right) \varrho_{y} \zeta \phi\right\|\|\psi\|=\mathcal{O}\left(a+\|\nabla \chi\|_{\infty}\right)\|\phi\|\|\psi\| .
\end{aligned}
$$

Since the remaining part of the integral over $\left\{|\eta|<\eta_{0}\right\}$ does not pose any further problem, we see altogether that the first integral in (121) is absolutely convergent and of order $\mathcal{O}\left(\|\nabla \chi\|_{\infty}+a\right)$.

Next, we treat the second integral on the right side of (121). Since we eventually have to change the gauge locally, we pick a (smooth) partition of unity, $\left\{J_{\nu}\right\}_{\nu \in \mathbb{Z}^{3}}$, on $\mathbb{R}^{3}$ such that $\operatorname{supp}\left(J_{\nu}\right) \subset \mathcal{B}_{1}(\nu)$, for every $\nu \in \mathbb{Z}^{3}$. We can certainly assume that $\sum_{\nu \in \mathbb{Z}^{3}}\left|\nabla J_{\nu}\right| \leqslant C$, for some $C \in(0, \infty)$. We set

$$
\mathscr{G}(\chi):=\left\{\nu \in \mathbb{Z}^{3}: J_{\nu} \chi \neq 0\right\}, \quad \mu:=\sum_{\nu \in \mathscr{G}(\chi)} J_{\nu}, \quad \bar{\mu}:=1-\mu,
$$


so that $\mu \chi=\chi, \bar{\mu} \chi=0$, and re-write the operator defined in (123) as

$$
\begin{aligned}
\widetilde{T} & =i \alpha \cdot(\chi \nabla F+\nabla \chi)+\chi\left[e^{F}, V_{\mathrm{E}}\right] e^{-F}+\left[\chi, V_{\mathrm{E}}\right] \\
& =\mu\left\{i \alpha \cdot(\chi \nabla F+\nabla \chi)+\chi\left[e^{F}, V_{\mathrm{E}}\right] e^{-F}+\left[\chi, V_{\mathrm{E}}\right]\right\}-\left\{\bar{\mu} V_{\mathrm{E}} \chi\right\} \mu \\
& =\quad \mu U_{1}-U_{2} \mu=\sum_{\nu \in \mathscr{G}(\chi)}\left(J_{\nu} U_{1}-U_{2} J_{\nu}\right) .
\end{aligned}
$$

For every $\nu \in \mathbb{Z}^{3}$, there is some gauge potential, $g_{\nu} \in C^{2}\left(\mathbb{R}^{3}, \mathbb{R}\right)$, such that $A-\nabla g_{\nu}=A_{\nu}$, where $A_{\nu}$ is defined by

$$
A_{\nu}(x):=\int_{0}^{1} B(\nu+t(x-\nu)) \wedge t(x-\nu) d t, \quad x \in \mathbb{R}^{3} .
$$

By virtue of (33) we obtain with $\widetilde{A}_{\nu}:=\nabla g_{\nu}$,

$$
\begin{aligned}
\varrho_{y} \bar{\zeta} & R_{A, V}(z) \widetilde{T}=\sum_{\nu \in \mathscr{G}(\chi)} \varrho_{y} \bar{\zeta} R_{A, V}(z)\left(J_{\nu} U_{1}-U_{2} J_{\nu}\right) \\
= & \sum_{\nu \in \mathscr{G}(\chi)} \varrho_{y} \bar{\zeta} R_{\widetilde{A}_{\nu}}(z)\left(J_{\nu} U_{1}-U_{2} J_{\nu}\right) \\
& +\sum_{\nu \in \mathscr{G}(\chi)} R_{\widetilde{A}_{\nu}}(z) i \alpha \cdot \nabla\left(\varrho_{y} \bar{\zeta}\right)\left(R_{\widetilde{A}_{\nu}}(z)-R_{A, V}(z)\right)\left(J_{\nu} U_{1}-U_{2} J_{\nu}\right) \\
& \quad-\sum_{\nu \in \mathscr{G}(\chi)} R_{\widetilde{A}_{\nu}}(z) \varrho_{y} \bar{\zeta}\left(V+\alpha \cdot A_{\nu}\right) R_{A, V}(z)\left(J_{\nu} U_{1}-U_{2} J_{\nu}\right) \\
=: & S_{1}+S_{2}-S_{3} .
\end{aligned}
$$

The proof of Proposition 3.11 is finished as soon as we have shown the following lemma.

A.2 Lemma. In the situation above there exists a $\chi$ - and $F$-independent constant, $C_{a_{0}} \in(0, \infty)$, such that, for $j=1,2,3$,

$$
\begin{aligned}
I_{j} & :=\int_{\Gamma}\left|\left\langle W_{y}^{1 / 2} \phi \mid S_{j} e^{F} R_{A, V}(z) e^{-F} \psi\right\rangle\right||d z| \\
& \leqslant C_{a_{0}}\left(1+\min \left\{|B(y)|,\|B\|_{\infty, \chi}\right\}\right)\left(\|\nabla \chi\|_{\infty}+a\right)\|\phi\|\|\psi\| .
\end{aligned}
$$

Proof of Lemma A.2: In our estimations below, that involve non-local operators, we exploit the fact that the interference between spatially seperated regions decays exponentially. Therefore, we start by introducing appropriate exponential weight functions: We pick some $\tilde{a} \in\left(\tau, \min \left\{\triangle_{0}, m\right\}\right)$ and a convex, 
even function $\tilde{f} \in C^{\infty}(\mathbb{R},[0, \infty))$ such that $\tilde{f} \equiv 0$ on $[-2,2], \tilde{f}(t)=\tilde{a}|t|-3 \tilde{a}$, for $|t| \geqslant 4$, and $0 \leqslant \tilde{f}^{\prime} \leqslant 1$ on $(0, \infty)$. We define $f_{\nu}(x):=\tilde{f}(|x-\nu|)$, $x \in \mathbb{R}^{3}$, so that $f_{\nu} \equiv 0$ on $\mathcal{B}_{2}(\nu)$ and $f_{\nu} \geqslant \tilde{a} \operatorname{dist}\left(\cdot, \mathcal{B}_{2}(\nu)\right)-\tilde{a}$ with equality outside $\mathcal{B}_{4}(\nu)$. Moreover, $\left|\nabla f_{\nu}\right| \leqslant \tilde{a}$. We further pick some non-decreasing $\theta \in C^{\infty}(\mathbb{R}, \mathbb{R})$ such that $\theta(t)=t$, for $t \leqslant 1, \theta \equiv 2$ on $[3, \infty)$ and $\theta^{\prime} \leqslant 1$. We set $\theta_{\nu, y}(t):=(|\nu-y|+1) \theta(t /(|\nu-y|+1)), t \in \mathbb{R}$, and $f_{\nu, y}:=\theta_{\nu, y} \circ f_{\nu}$, so that $f_{\nu, y}$ is bounded, $f_{\nu, y}=f_{\nu} \geqslant \tilde{a} \operatorname{dist}\left(\cdot, \mathcal{B}_{2}(\nu)\right)-\tilde{a}$ on $\mathcal{B}_{1}(y) \supset \operatorname{supp}\left(\varrho_{y}\right)$, and $\left|\nabla f_{\nu, y}\right| \leqslant \tilde{a}$. By construction $e^{f_{\nu, y}} J_{\nu}=J_{\nu}$. Setting

$$
\widetilde{\psi}_{\nu, y}(z):=\left(J_{\nu} U_{1}-e^{f_{\nu, y}} U_{2} e^{-f_{\nu, y}} J_{\nu}\right) e^{F} R_{A, V}(z) e^{-F} \psi
$$

we thus have

$$
\left(J_{\nu} U_{1}-U_{2} J_{\nu}\right) e^{F} R_{A, V}(z) e^{-F} \psi=e^{-f_{\nu, y}} \widetilde{\psi}_{\nu, y}(z) .
$$

Observing that $\bar{\mu} \chi=0$ implies

$$
e^{f_{\nu, y}} U_{2} e^{-f_{\nu, y}}=\bar{\mu}\left[e^{f_{\nu, y}} V_{\mathrm{E}} e^{-f_{\nu, y}}, \chi\right]
$$

and employing (11) and (38) we further find some constant $C \in(0, \infty)$ such that, for all $z=e_{0}+i \eta \in \Gamma, \nu \in \mathbb{Z}^{3}$, and $y \in \mathbb{R}^{3}$,

$$
\left\|\widetilde{\psi}_{\nu, y}(z)\right\| \leqslant C \frac{a+\|\nabla \chi\|_{\infty}}{\sqrt{1+\eta^{2}}}\|\psi\|
$$

Taking these remarks into account we obtain

$$
I_{1} \leqslant \sum_{\nu \in \mathscr{G}(\chi)} \int_{\Gamma}\left|\left\langle e^{-f_{\nu, y}} R_{\widetilde{A}_{\nu}}(\bar{z}) e^{f_{\nu, y}} W_{y}^{1 / 2}\left(\varrho_{y} e^{-f_{\nu, y}}\right) \bar{\zeta} \phi \mid \widetilde{\psi}_{\nu, y}(z)\right\rangle\right||d z|
$$

Now, since $\widetilde{A}_{\nu}=\nabla g_{\nu}$ is a gradient we have curl $\widetilde{A}_{\nu}=0$, whence (6), (17), and Hardy's inequality imply

$$
\left\|W_{y} \varphi\right\| \leqslant C\left\|\nabla\left(e^{i g_{\nu}} \varphi\right)\right\|=C\left\|\left(-i \nabla+\widetilde{A}_{\nu}\right) \varphi\right\| \leqslant C\left\|\left|D_{\widetilde{A}_{\nu}}\right| \varphi\right\|,
$$

for $\varphi \in \mathscr{D}$, with some $\nu$ - and $y$-independent constant $C \in(0, \infty)$. Standard arguments now imply that $\left|D_{\widetilde{A}_{\nu}}\right|^{-1 / 2} W_{y}^{1 / 2}$ is a bounded operator whose norm is uniformly bounded in $\nu$ and $y$. Setting

$$
\phi_{\nu}:=\left|D_{\widetilde{A}_{\nu}}\right|^{-1 / 2} W_{y}^{1 / 2}\left(\varrho_{y} e^{-f_{\nu, y}}\right) \bar{\zeta} \phi
$$


and applying (45) we thus find

$$
\begin{aligned}
& I_{1} \leqslant \sum_{\nu \in \mathscr{G}(\chi)}\left(\int_{\Gamma}\left\|e^{-f_{\nu, y}} R_{\widetilde{A}_{\nu}}(\bar{z}) e^{f_{\nu, y}}\left|D_{\widetilde{A}_{\nu}}\right|^{1 / 2} \phi_{\nu}\right\|^{2}|d z|\right)^{1 / 2} \\
& \cdot\left(\int_{\Gamma}\left\|\widetilde{\psi}_{\nu, y}(z)\right\|^{2}|d z|\right)^{1 / 2} \\
& \leqslant C \sum_{\nu \in \mathscr{G}(\chi)}\left\|\phi_{\nu}\right\|\left(a+\|\nabla \chi\|_{\infty}\right)\left(\int_{\mathbb{R}} \frac{d \eta}{1+\eta^{2}}\right)^{1 / 2}\|\psi\| \\
& \leqslant C^{\prime} \sum_{\nu \in \mathbb{Z}^{3}} \sup \left\{e^{-f_{\nu, y}(x)}: x \in \mathcal{B}_{1}(y)\right\}\|\phi\|\left(a+\|\nabla \chi\|_{\infty}\right)\|\psi\| \\
& \leqslant C^{\prime \prime}\left(a+\|\nabla \chi\|_{\infty}\right)\|\phi\|\|\psi\| .
\end{aligned}
$$

For $I_{2}$, we obtain the estimate by means of (150), (38), and (130),

$$
\begin{aligned}
I_{2} \leqslant & \sum_{\nu \in \mathscr{G}(\chi)} \int_{\Gamma}|d z|\left\|\left|D_{\widetilde{A}_{\nu}}\right|^{-1 / 2} W_{y}^{1 / 2}\right\|\|\phi\|\left\|\left|D_{\widetilde{A}_{\nu}}\right|^{1 / 2} R_{\widetilde{A}_{\nu}}(z)\right\| \\
& \cdot\left\|\left|\nabla\left(\varrho_{y} \bar{\zeta}\right)\right| e^{-f_{\nu, y}}\right\|\left\|e^{f_{\nu, y}}\left(R_{A, V}(z)-R_{\widetilde{A}_{\nu}}(z)\right) e^{-f_{\nu, y}}\right\|\left\|\widetilde{\psi}_{\nu, y}(z)\right\| \\
\leqslant & C\left(\|\nabla \chi\|_{\infty}+a\right) \sum_{\nu \in \mathbb{Z}^{3}} \sup \left\{e^{-f_{\nu, y}(x)}: x \in \mathcal{B}_{1}(y)\right\}\|\phi\|\|\psi\| \\
\leqslant & C^{\prime}\left(\|\nabla \chi\|_{\infty}+a\right)\|\phi\|\|\psi\| .
\end{aligned}
$$

To derive a bound on $I_{3}$ we employ the special properties of the gauge transformed vector potentials $A_{\nu}$. Namely, we make use of the bound

$\varrho_{y}(x) e^{-f_{\nu, y}(x)}\left|A_{\nu}(x)\right| \leqslant \varrho_{y}(x) \frac{|x-\nu|}{2}\left(b_{1} e^{-(\tilde{a}-\tau)|x-\nu|+3 \tilde{a}}+|B(\nu)| e^{-\tilde{a}|x-\nu|+3 \tilde{a}}\right)$,

for all $\nu \in \mathbb{Z}^{3}$ and $x \in \mathbb{R}^{3}$, which follows from (23). Since also $|B(\nu)| \leqslant$ $|B(y)|+|B(\nu)-B(y)|$ and $|x-y| \leqslant 1$, if $\varrho_{y}(x) \neq 0$, we infer again from (23) that

$$
\begin{aligned}
& \sum_{\nu \in \mathscr{G}(\chi)}\left\|\varrho_{y} e^{-f_{\nu, y}}\left|A_{\nu}\right|\right\|_{\infty} \\
& \leqslant C \sum_{\nu \in \mathscr{G}(\chi)} e^{-\hat{a}|y-\nu|}\left(1+\min \left\{|B(y)|, \sup _{\nu \in \mathscr{G}(\chi)}|B(\nu)|\right\}\right),
\end{aligned}
$$

for some sufficiently small $\hat{a}>0$. Using these observations and the uniform boundedness of $\bar{\zeta} e^{f_{\nu, y}} V e^{-f_{\nu, y}}$, which is implied by Hypothesis 2 and the choice 
of $\zeta$, we find some $\chi^{-}, F$-, and $y$-independent constant $C^{\prime} \in(0, \infty)$ such that

$$
\begin{aligned}
I_{3} \leqslant & \sum_{\nu \in \mathscr{G}(\chi)} \int_{\Gamma}\left\|R_{\widetilde{A}}(\bar{z}) W_{y}^{1 / 2}\right\|\|\phi\|\left\{\left\|\varrho_{y} e^{-f_{\nu, y}}\right\|_{\infty}\left\|\bar{\zeta} e^{f_{\nu, y}} V e^{-f_{\nu, y}}\right\|\right. \\
& \left.+\left\|\varrho_{y} e^{-f_{\nu, y}}\left|A_{\nu}\right|\right\|_{\infty}\right\}\left\|e^{f_{\nu, y}} R_{A, V}(z) e^{-f_{\nu, y}}\right\|\left\|\widetilde{\psi}_{\nu, y}(z)\right\||d z| \\
& \leqslant C^{\prime}\left(1+\min \left\{|B(y)|,\|B\|_{\infty, \chi}\right\}\right)\left(\|\nabla \chi\|_{\infty}+a\right)\|\phi\|\|\psi\| .
\end{aligned}
$$

This completes the proof of Lemma A.2 and, at the same time, the proof of Proposition 3.11. (The last assertion of Proposition 3.11 follows by inspecting the arguments above.)

Proof of Lemma 3.13: We use the notation introduced in the proofs of Proposition 3.11 and Lemma A.2 in the following. We already know from Corollary 3.9 that the vector $\Lambda_{A, V}^{+} \psi$ belongs to $\mathcal{D}\left(W_{y}^{1 / 2}\right)$, but we do not have any control on the norm on the left in (66) yet. It is certainly sufficient to derive the asserted bound with $W_{y}^{1 / 2}$ replaced by $\varrho_{y} W_{y}^{1 / 2}$. As in the proof of Corollary 3.9 we first pick some $\zeta \in C_{0}^{\infty}\left(\mathbb{R}^{3},[0,1]\right)$ (independent of $\psi$ ) such that $\zeta \equiv 1$ on some large open ball containing $\mathcal{Y}$ and set $\bar{\zeta}=1-\zeta$. By the closed graph theorem $\left|D_{0}\right|^{1 / 2} \zeta R_{A, V}(i)$ is bounded whence

$$
\left\|\varrho_{y} W_{y}^{1 / 2} \zeta \Lambda_{A, V}^{+} \psi\right\| \leqslant C\left\|\left|D_{0}\right|^{1 / 2} \zeta R_{A, V}(i)\right\|\left\|\left(D_{A, V}-i\right) \psi\right\| .
$$

We denote the characteristic function of the support of $\psi$ by $\chi$. To treat the remaining piece of the norm we set

$$
\widetilde{\psi}_{\nu}:=\Lambda_{A, V}^{+}\left(D_{A, V}-i\right) J_{\nu} \psi,
$$

and write analogously to (129),

$$
\begin{aligned}
& \left|\left\langle W_{y}^{1 / 2} \phi \mid \varrho_{y} \bar{\zeta} \Lambda_{A, V}^{+} \psi\right\rangle\right| \leqslant \sum_{\nu \in \mathscr{G}(\chi)}\left|\left\langle W_{y}^{1 / 2} \phi \mid \varrho_{y} \bar{\zeta} R_{A, V}(i) \widetilde{\psi}_{\nu}\right\rangle\right| \\
& \leqslant \quad \sum_{\nu \in \mathscr{G}(\chi)}\left|\left\langle W_{y}^{1 / 2} \varrho_{y} \bar{\zeta} \phi \mid R_{\widetilde{A}_{\nu}}(i) \widetilde{\psi}_{\nu}\right\rangle\right| \\
& \quad+\sum_{\nu \in \mathscr{G}(\chi)}\left|\left\langle W_{y}^{1 / 2} \phi \mid R_{\widetilde{A}_{\nu}}(i) \alpha \cdot \nabla\left(\varrho_{y} \zeta\right)\left(R_{A, V}(i)-R_{\widetilde{A}_{\nu}}(i)\right) \widetilde{\psi}_{\nu}\right\rangle\right| \\
& \quad+\sum_{\nu \in \mathscr{G}(\chi)}\left|\left\langle W_{y}^{1 / 2} \phi \mid R_{\widetilde{A}_{\nu}}(i) \varrho_{y} \bar{\zeta}\left(V+\alpha \cdot A_{\nu}\right) R_{A, V}(i) \widetilde{\psi}_{\nu}\right\rangle\right| \\
& =: \quad Q_{1}+Q_{2}+Q_{3},
\end{aligned}
$$


where $\phi \in H^{1 / 2}\left(\mathbb{R}^{3}, \mathbb{C}^{4}\right)$. Again we use exponential weights constructed in the beginning of the proof of Lemma A.2 and abbreviate

$$
\widehat{\psi}_{\nu, y}:=\left(e^{f_{\nu, y}} \Lambda_{A, V}^{+} e^{-f_{\nu, y}}\right) e^{f_{\nu, y}}\left(D_{A, V}-i\right) e^{-f_{\nu, y}} J_{\nu} \psi,
$$

so that by Corollary 3.7

$$
\left\|\widehat{\psi}_{\nu, y}\right\| \leqslant C\left\|\left(D_{A, V}-i\right) \psi\right\|+\mathcal{O}\left(\left\|\nabla J_{\nu}\right\|_{\infty}+\tilde{a}\right)\|\psi\| \leqslant C^{\prime}\left\|\left(D_{A, V}-i\right) \psi\right\|,
$$

where $C, C^{\prime} \in(0, \infty)$ neither depend on $\nu$ nor $y$. Using also (40) we thus obtain

$$
\begin{aligned}
Q_{1} \leqslant & \sum_{\nu \in \mathscr{G}(\chi)}\left\|\left|D_{\widetilde{A}_{\nu}}\right|^{-1 / 2} W_{y}^{1 / 2}\right\|\left\|\varrho_{y} e^{-f_{\nu, y}}\right\|\|\phi\| \\
& \cdot\left\|\left|D_{\widetilde{A}_{\nu}}\right|^{1 / 2} e^{f_{\nu, y}} R_{\widetilde{A}_{\nu}}(i) e^{-f_{\nu, y}}\right\|\left\|\widehat{\psi}_{\nu, y}\right\| \\
\leqslant & C^{\prime \prime}\|\phi\|\left\|\left(D_{A, V}-i\right) \psi\right\| .
\end{aligned}
$$

Using (132) we further find

$$
\begin{aligned}
Q_{3} \leqslant & \sum_{\nu \in \mathscr{G}(\chi)}\left\|\left|D_{\widetilde{A}_{\nu}}\right|^{-1 / 2} W_{y}^{1 / 2}\right\|\|\phi\|\left\|\left|D_{\widetilde{A}_{\nu}}\right|^{1 / 2} R_{\widetilde{A}_{\nu}}(i)\right\| \\
& \cdot\left\{\left\|\varrho_{y} e^{-f_{\nu, y}}\right\|_{\infty}\left\|\bar{\zeta} e^{f_{\nu, y}} V e^{-f_{\nu, y}}\right\|+\left\|\varrho_{y} e^{-f_{\nu, y}}\left|A_{\nu}\right|\right\|_{\infty}\right\}\left\|\widehat{\psi}_{\nu, y}\right\| \\
\leqslant & C^{\prime \prime \prime}\left(1+\min \left\{|B(y)|,\|B\|_{\infty, \psi}\right\}\right)\|\phi\|\left\|\left(D_{A, V}-i\right) \psi\right\| .
\end{aligned}
$$

The remaining term, $Q_{2}$, can be dealt with similarly.

Acknowledgement: It is a pleasure to thank Hubert Kalf, Sergey Morozov, and Heinz Siedentop for useful remarks and helpful discussions. Moreover, we thank Sergey Morozov for making parts of his manuscripts [29] available to us prior to publication.

\section{References}

[1] V. Bach, J. Fröhlich, and I. Sigal. Quantum electrodynamics of confined non-relativistic particles. Adv. Math., 137: 299-395, 1998.

[2] V. Bach and O. Matte. Exponential decay of eigenfunctions of the BetheSalpeter operator. Lett. Math. Phys., 55: 53-62, 2001.

[3] A. A. Balinsky and W. D. Evans. On the virial theorem for the relativistic operator of Brown and Ravenhall, and the absence of embedded eigenvalues. Lett. Math. Phys., 44: 233-248, 1998. 
[4] A. A. Balinsky and W. D. Evans. Stability of one-electron molecules in the Brown-Ravenhall model. Comm. Math. Phys., 202: 481-500, 1999.

[5] A. A. Balinsky and W. D. Evans. On the spectral properties of the BrownRavenhall operator. J. Comput. Appl. Math., 148: 239-255, 2002.

[6] A. Berthier and V. Georgescu. On the point spectrum of Dirac operators. J. Funct. Anal., 71: 309-338, 1987.

[7] H. A. Bethe and E. E. Salpeter. Quantum mechanics of one- and twoelectron atoms. In: Handbuch der Physik, XXXV. (Pages 88-436.) Edited by S. Flügge. Springer, Berlin, 1957.

[8] A. M. Boutet de Monvel and R. Purice. A distinguished self-adjoint extension for the Dirac operator with strong local singularities and arbitrary behaviour at infinity. Rep. Math. Phys., 34: 351-360, 1994.

[9] G. E. Brown and D. G. Ravenhall. On the interaction of two electrons. Proc. Roy. Soc. London A, 208: 552-559, 1951.

[10] R. Cassanas and H. Siedentop The ground-state energy of heavy atoms according to Brown and Ravenhall: absence of relativistic effects in leading order. J. Phys. A, 39: 10405-10414, 2006.

[11] H. L. Cycon, R. G. Froese, W. Kirsch, and B. Simon. Schrödinger operators. Texts and Monographs in Physics, Springer, Berlin-Heidelberg, 1987.

[12] M. Dimassi and J. Sjöstrand. Spectral asymptotics in the semi-classical limit. London Math. Soc. Lecture Note Series, 268. Cambridge University Press, Cambridge, 1999.

[13] J. Dolbeault, M. J. Esteban, and M. Loss. Relativistic hydrogenic atoms in strong magnetic fields. Preprint, 2006.

[14] W. D. Evans, P. Perry and H. Siedentop. The spectrum of relativistic one-electron atoms according to Bethe and Salpeter. Comm. Math. Phys., 178: 733-746, 1996.

[15] V. Georgescu and M. Măntoiu. On the spectral theory of singular Dirac type Hamiltonians. J. Operator theory, 46: 289-321, 2001.

[16] M. Griesemer. Exponential decay and ionization thresholds in nonrelativistic quantum electrodynamics. J. Funct. Anal., 210: 321-340, 2004. 
[17] M. Griesemer and H. Siedentop. A minimax principle for the eigenvalues in spectral gaps. J. London Math. Soc. (2), 60: 490-500, 1999.

[18] M. Griesemer and C. Tix. Instability of a pseudo-relativistic model of matter with self-generated magnetic field. J. Math. Phys., 40: 1780-1791, 1999.

[19] C. Hainzl, M. Lewin, E. Séré, and J. P. Solovej. A minimization method for relativistic electrons in a mean-field approximation of quantum electrodynamics. Preprint, arXiv:0706.1486v1, 18 Pages, 2007.

[20] B. Helffer, J. Nourrigat, and X. P. Wang. Sur le spectre de l'équation de Dirac (dans $\mathbb{R}^{3}$ ou $\mathbb{R}^{2}$ ) avec champ magnetic. Ann. Sci. École Normale Superieur, 4e Série, 22: 515-533, 1989.

[21] G. Hoever and H. Siedentop. Stability of the Brown-Ravenhall operator. Math. Phys. Electr. J., 5: Paper 6, 11 pages, 1999.

[22] D. H. Jakubaßa-Amundsen. The HVZ theorem for a pseudo-relativistic operator. Ann. Henri Poincaré, 8: 337-360, 2007.

[23] D. H. Jakubaßa-Amundsen. The HVZ theorem for the Brown-Ravenhall operator with constant magnetic field. Preprint, arXiv:0709.1034, 27 Pages, 2007.

[24] T. Kato. Perturbation theory for linear operators. Classics in mathematics, Springer, Berlin-Heidelberg, 1995.

[25] T. Kato. Holomorphic families of Dirac operators. Math. Z., 183: 399-406, 1983.

[26] E. H. Lieb and M. Loss. Stability of a model of relativistic quantum electrodynamics. Comm. Math. Phys., 228: 561-588, 2002.

[27] E. H. Lieb, H. Siedentop, and J. P. Solovej. Stability and instability of relativistic electrons in classical electromagnetic fields. J. Statist. Phys., 89: 37-59, 1997.

[28] S. Morozov. Essential spectrum of multiparticle Brown-Ravenhall operators in external field. Preprint, arXiv:0802.0453, 29 Pages, 2008.

[29] S. Morozov. PhD thesis, Universität München. In preparation.

[30] S. Morozov and S. Vugalter. Stability of atoms in the Brown-Ravenhall model. Ann. Henri Poincaré, 7: 661-687, 2006. 
[31] G. Nenciu. Self-adjointness and invariance of the essential spectrum for Dirac operators defined as quadratic forms. Comm. Math. Phys., 48: 235$247,1976$.

[32] G. Nenciu. Distinguished self-adjoint extension for the Dirac operator with potentia dominated by multicenter Coulomb potentials. Helvetica Phys. Acta, 50: 1-3, 1977.

[33] R. Richard and R. Tiedra de Aldecoa. On the spectrum of magnetic Dirac operators with Coulomb-type perturbations. Preprint, arXiv: math-ph/0611072v1, 2006.

[34] J. Sucher. Foundations of the relativistic theory of many-electron atoms. Phys. Rev. A, 22: 348-362, 1980.

[35] J. Sucher. Relativistic many-electron Hamiltonians. Physica Scripta, 36: 271-281, 1987.

[36] B. Thaller. The Dirac equation. Texts and Monographs in Physics, Springer, Berlin-Heidelberg, 1992.

[37] C. Tix. Strict positivity of a relativistic Hamiltonian due to Brown and Ravenhall. Bull. London Math. Soc., 30: 283-290.

[38] C. Tix. Self-adjointness and spectral properties of a pseudo-relativistic Hamiltonian due to Brown and Ravenhall. Preprint, mp_arc 97-441, 20 pages, 1997.

[39] C. Tix. Lower bound for the ground state energy of the no-pair Hamiltonian. Phys. Lett. B, 405: 293-296, 1997.

[40] J. Xia. On the contribution of the Coulomb singularity of arbitrary charge to the Dirac Hamiltonian. Trans. Amer. Math. Soc., 351: 1989-2023. 